\title{
The restaurant at the end of the random walk: Recent developments in the description of anomalous transport by fractional dynamics.
}

\author{
Ralf Metzler ${ }^{\sharp}$ and Joseph Klafter ${ }^{\dagger}$ \\ \# NORDITA, Blegdamsvej 17, DK-2100 Copenhagen Ø, Denmark \\ †School of Chemistry, Tel Aviv University, Ramat Aviv, 69978 Tel Aviv, Israel \\ E-mail: metz@nordita.dk,klafter@post.tau.ac.il
}

\begin{abstract}
Fractional dynamics has experienced a firm upswing during the last years, having been forged into a mature framework in the theory of stochastic processes. A large number of research papers developing fractional dynamics further, or applying it to various systems have appeared since our first review article on the fractional FokkerPlanck equation [R. Metzler and J. Klafter, Phys. Rep. 339 (2000) 1-77]. It therefore appears timely to put these new works in a cohesive perspective. In this review we cover both the theoretical modelling of sub- and superdiffusive processes, placing emphasis on superdiffusion, and the discussion of applications such as the correct formulation of boundary value problems to obtain the first passage time density function. We also discuss extensively the occurrence of anomalous dynamics in various fields ranging from nanoscale over biological to geophysical and environmental systems.
\end{abstract}

PACS numbers: 02.50.-r,05.40.-a,82.37.-j,87.15.-v

\section{Introduction}

For seven and a half million years, Deep Thought computed and calculated, and in the end announced that the answer was in fact Forty-two-and so another, even bigger, computer had to be built to find out what the actual question was. $\neq$

The notions and concepts of anomalous dynamical properties, such as long-range spatial or temporal correlations manifested in power-laws, stretched exponentials, $1 / f^{\alpha_{-}}$ noises, or non-Gaussian probability density functions (PDFs), have been predicted and observed in numerous systems from various disciplines including physics, chemistry, engineering, geology, biology, economy, meteorology, astrophysics and others. Apart from other standard tools to describe anomalous dynamics such as continuous time random walks [Blumen et al (1986), Bouchaud and Georges (1990), Hughes (1995),

$\ddagger$ Douglas Adams, The Restaurant at the End of the Universe, Tor Books, 1988 
Klafter et al (1996), Shlesinger et al (1993)], fractional dynamical equations have become increasingly popular to model anomalous transport [Barkai (2001), Hilfer (2000), Metzler and Klafter (2000), Metzler and Klafter (2001), Sokolov et al (2002)]. In the presence of an external force field, in particular, the fractional Fokker-Planck equation provides a direct extension of the classical Fokker-Planck equation, being amenable to well-known methods of solution.

This review updates and complements with new and different perspectives the 'Random walk's guide to anomalous diffusion' [Metzler and Klafter (2000)]. Since its publication, a large volume of research covering recent developments in the fractional dynamics framework and its applications has been conducted, most of which is brought together herein. We refrain from a repetition of the historical context and the mathematical details presented in [Metzler and Klafter (2000), Metzler and Klafter (2001)], and we build on the material and notations introduced there. What we wish to point out is the framework character: Just as the regular Fokker-Planck equation renders itself to the description of a plethora of processes, so does the fractional analogue in all those systems whose statistics is governed by the ubiquitous power-laws. The breadth of such potential applications, at the same time, may indeed encourage the usage in many new fields.

We start with a collection of systems, in which have been observed anomalous processes with long-range correlations, covering both experimental and theoretical evidence. Having set the scene, we divide the introduction of fractional dynamics concepts between subdiffusive and superdiffusive processes, and for the latter we distinguish between Lévy flights and walks [Klafter et al (1996), Shlesinger et al (1993)]. Finally, we discuss various applications of the framework, in particular, the formulation and solution of first passage time problems and fractional diffusion-reaction processes. In the appendix, we collect some important concepts and definitions on fractional operators and their physical origin.

\section{Processes of anomalous nature}

As already mentioned, processes deviating from the classical Gaussian diffusion or exponential relaxation patterns occur in a multitude of systems. The anomalous features usually stretch over the entire data window, but there exist examples when they develop after an initial period of sampling (finite size/time effects), or they may be transient, i.e., eventually the anomalous process nature turns into normal transport or relaxation dynamics. In the anomalous regime, possibly the most fundamental definition of anomaly of the form we have in mind is the deviation of the mean squared displacement

$$
\left\langle(\Delta \mathbf{r})^{2}\right\rangle=\left\langle(\mathbf{r}-\langle\mathbf{r}\rangle)^{2}\right\rangle=2 d K_{\alpha} t^{\alpha}
$$

from the 'normal' linear dependence $\left\langle(\Delta \mathbf{r})^{2}\right\rangle=2 d K_{1} t$ on time. Here, $d$ is the (embedding) spatial dimension, and $K_{1}$ and $K_{\alpha}$ are the normal and generalised diffusion constants of dimensions $\mathrm{cm}^{2} / \mathrm{sec}$ and $\mathrm{cm}^{2} / \mathrm{sec}^{\alpha}$, respectively. The anomalous diffusion 
exponent $\alpha \neq 1$ determines whether the process will be categorised as subdiffusive (dispersive, slow, etc.) if $0<\alpha<1$, or superdiffusive (enhanced, fast) if $1<\alpha$. Usually, the domain $1<\alpha \leq 2$ is considered, $\alpha=2$ being the ballistic limit described by the wave equation, or its forward and backward modes [Landau and Lifshitz (1984)].§ Processes with $\alpha>2$ are known, such as the Richardson pair diffusion in fully developed turbulence [Richardson (1926)]. However, we will restrict our discussion to sub-ballistic processes with $\alpha<2$ explicitly.\| An exception of equation (1) are unconfined Lévy flights, for which we observe a diverging mean squared displacement. We will concentrate on the one-dimensional case, to keep notations simple, in particular, for the case of Lévy flights.

Before we continue, we stop to highlight the parallels and main differences between fractional dynamics and other dynamical models, as compiled in table 1 for forcefree anomalous diffusion. Thus, Brownian motion (BM) can be generalised using the continuous time random walk (CTRW) model to subdiffusion or dispersive transport (SD), to Lévy flights (LF), or to Lévy walks (LW), see the table caption for more details. All of these models can be mapped onto the corresponding fractional equations, as discussed in the following sections. These descriptions differ from fractional Brownian motion (FBM) or the generalised Langevin equation (GLE). Despite from being Gaussian in nature such as the PDF in Brownian dynamics, fractional Brownian motion, and generalised Langevin equation descriptions, the subdiffusion PDF has an asymptotic stretched Gaussian shape, Lévy flights are characterised by a long-tailed Lévy stable law, and Lévy walks exhibit spikes of finite propagation velocity, in between which an approximate Lévy stable PDF is being spanned continuously. As we will see from the fractional dynamical equations corresponding to SD, LFs, and LWs, they are highly non-local, and carry far-reaching correlations in time and/or space, represented in the integro-differential nature (with slowly decaying power-law kernels) of these equations. In contrast, FBM and GLE on the macroscopic level are local in space and time, and carry merely time- or space-dependent coefficients. We also note that anomalous diffusion can be modelled in terms of non-linear Fokker-Planck equations based on nonextensive statistical approaches [Borland (1998)]. However, we intend to consider linear equations in what follows.

To build our case, let us continue by presenting a list of examples from different areas for which the anomalous character has been demonstrated.

$\S$ Similarly, the diffusion equation can be rewritten with a half-order derivative in time [Oldham and Spanier (1972)].

|| It was shown that the restriction to sub-ballistic motion guarantees for all non-pathological processes fulfilling equation (1) that the fluctuation-dissipation theorem holds [Costa et al (2003), Morgado et al (2002)].

ฯ As will be discussed below, in the presence of steep external potentials, the mean squared displacement of Lévy flights becomes finite. 
Table 1. Comparison of different anomalous diffusion models to normal Brownian motion (BM) [Lévy (1965), van Kampen (1981)]: PDFs of fractional Brownian motion (FBM) [Mandelbrot and van Ness (1968), Lim and Muniandy (2002), Lutz (2001a)], generalised Langevin equation with power-law kernel (GLE) [Kubo et al (1985), Lutz (2001a), Wang et al (1994), Wang and Tokuyama (1999)]; continuous time random walk (CTRW) of types subdiffusion (SD), Lévy flights (LF) and Lévy walks [Klafter et al (1987), Klafter et al (1996), Shlesinger et al (1993)]; as well as time-fractional dynamics (TFD), which covers both subdiffusion (in this case it corresponds to SD) and sub-ballistic superdiffusion [Metzler and Klafter (2000), Metzler and Klafter (2000c)]. The $c_{i}$ are constants.

${ }^{a}$ Note that there are various definitions of FBM. However, the Gaussian nature is common to all versions. The behaviour of FBM is antipersistent for $0<\alpha<1$, and persistent for $1<\alpha \leq 2$ [Mandelbrot (1982)].

${ }^{b}$ The GLE is in some sense more fundamental than the FBM. For instance, it occurs naturally hydrodynamic backflow [Kubo et al (1985), Landau and Lifshitz (1987)], and generally includes an external force. The case $\alpha=1$ leads to a logarithmic correction of the form $\left\langle x^{2}(t)\right\rangle \sim t \log t$ in the GLE-formulation chosen in reference [Wang and Tokuyama (1999)].

${ }^{c}$ Same (asymptotic) PDF as in the TFD case with $0<\alpha \leq 1$.

${ }^{d}$ The symmetric Lévy stable law of index $\mu$, with diverging variance $\left\langle x^{2}(t)\right\rangle=\infty$. LFs correspond to the space-fractional diffusion equation (33).

${ }^{e}$ For appropriate exponents $\mu$ and $\nu$, LWs are identical to the SD and the FD, while for subdiffusion, they exhibit spikes spreading with finite velocity, continuously spanning a Lévy stable-like propagator between them [Klafter and Zumofen (1994)]. Superdiffusive LWs are described in terms of the fractional material derivative (54). ${ }^{f}$ Stretched $(0<\alpha<1)$ and compressed $(1<\alpha<2)$ Gaussian governed by equation (9).

\begin{tabular}{|c|c|c|}
\hline & $\mathrm{PDF}$ & Comments \\
\hline BM & $P(x, t)=(4 \pi K t)^{-1 / 2} \exp \left(-x^{2} /(4 K t)\right)$ & \\
\hline FBM & $P(x, t)=\left(4 \pi K_{\alpha} t^{\alpha}\right)^{-1 / 2} \exp \left(-x^{2} /\left(4 K_{\alpha} t^{\alpha}\right)\right)$ & $0<\alpha \leq 2^{a}$ \\
\hline GLE & $P(x, t)=\left(4 \pi K_{\alpha} t^{\alpha}\right)^{-1 / 2} \exp \left(-x^{2} /\left(4 K_{\alpha} t^{\alpha}\right)\right)$ & $0<\alpha<2, \alpha \neq 1^{b}$ \\
\hline SD & $\begin{aligned} P(x, t) & \sim c_{1} t^{-\alpha / 2} \xi^{-(1-\alpha) /(2-\alpha)} \exp \left(-c_{2} \xi^{1 /(1-\alpha / 2)}\right) \\
\xi & \equiv|x| / t^{\alpha}\end{aligned}$ & $0<\alpha \leq 1^{c}$ \\
\hline $\mathrm{LF}$ & $P(x, t)=f^{-1}\left\{\exp \left(-K^{\mu} t|x|^{\mu}\right)\right\} \sim K^{\mu} t /|x|^{1+\mu}$ & $0<\mu \leq 2^{d}$ \\
\hline LW & $\begin{array}{c}P(k, u)=\frac{1}{u} \psi(u) /[1-\psi(k, u)] \therefore \psi(t) \sim \tau^{\alpha} / t^{1+\alpha} \\
\psi(x, t)=\frac{1}{2}|x|^{-\mu} \delta\left(|x|-v_{\nu} t^{\nu}\right)\end{array}$ & $\nu \mu>1^{e}$ \\
\hline TFD & $\begin{array}{c}P(x, t) \sim c_{1} t^{-\alpha / 2} \xi^{-(1-\alpha) /(2-\alpha)} \exp \left(-c_{2} \xi^{1 /(1-\alpha / 2)}\right) \\
\xi \equiv|x| / t^{\alpha}\end{array}$ & $0<\alpha<2^{f}$ \\
\hline
\end{tabular}




\subsection{Geophysical and geological processes}

The seasonal variations of rivers, and the water balance in general, has been studied extensively over many decades, in particular, due to its environmental importance. Thus, for the water discharge variations of Lake Albert during his studies of the time-variations of the Nile river, Hurst found that they cannot fall into the class of statistically independent processes, but can only be explained by a process, which is correlated in time. Similar effects he reported on rainfall statistics, and tree rings [Hurst (1951), Hurst et al (1951), Feder (1988)]. More recently, draught- and rainduration as well as rain-size of localised rain events have in fact been confirmed to obey power-law statistics [Dickman (2003), Peters et al (2002)], which also enter earthquake aftershocks dynamics [Helmstetter and Sornette (2002)]. But also the 'products' of dynamical processes are often non-trivial, such as the fractal nature of coastline, also known as the 'Coastline-of-Britain' phenomenon based on data collected by Richardson [Mandelbrot (1967)], or the anomalous scaling between drainage area and river network length discovered originally by Hack [Hack (1957)].

Considerable attention is paid to the investigation of tracer diffusion in subsurface hydrology, primarily for its obvious environmental implications. Thus, large scale field experiments were undertaken, such as at the Borden site in Ontario, Canada [Sudicky (1986)], at Cape Cod, Massachusetts [LeBlanc et al (1991)], or during the MAcro-Dispersion Experiment (MADE) at Columbus Air Force Base, Mississippi [Boggs et al (1993), Adams and Gelhar (1992), Rehfeldt et al (1992)], indicating that tracer dispersion is controlled by strong non-locality causing highly non-Gaussian PDFs (in this context often called plumes) seen as "scale-dependent dispersion" [Gelhar et al (1992)]. It has been shown that long-tailed waiting time distributions with a comparably small number of fit parameters can well account for the observed behaviour [Berkowitz et al (2002), Berkowitz and Scher (1995), Berkowitz and Scher (1997), Scher et al (2002a)]; however, also space-fractional models were used to account for the anomalies [Benson et al (2001)]. In a similar study, longtime catchment data of chloride tracer in rainwater recorded in Wales, UK, were shown to follow $1 / f$ statistics in the power spectrum [Kirchner et al (2000)], which might indicate strongly non-local correlations in time, which was later interpreted from an anomalous dynamics point of view, indicating that the data are perfectly consistent with a power-law form for the sticking time distribution of tracer particles in the catchment, causing extremely long retention times [Scher et al (2002)]. Any contaminant getting into an aquifer fostering such anomalous dynamics, that is, will take considerably longer to leave the aquifer than the advecting water, in which it diffuses. This is, for instance, illustrated in figure 1, contrasting the drift-dominated behaviour of the water with the tracer outflow. According to the modelling brought forth in reference [Scher et al (2002)], the mean retention time for the tracer becomes infinite, and is possibly due to sticking effects or trapping of the tracer in side-channels off the aquifer backbone. We note that even on the laboratory-scale, fairly simple systems were found 

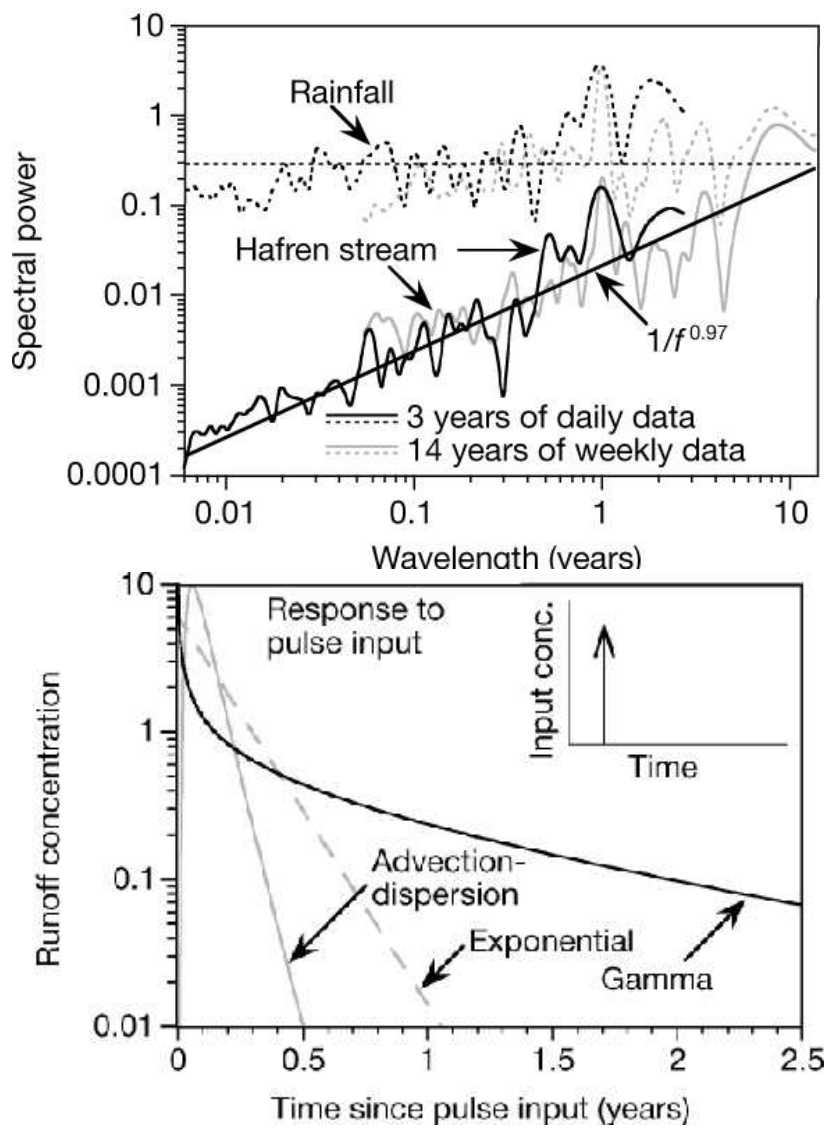

Figure 1. Top: Power spectra of chloride tracer originally contained in rainwater, and as measured at the outflow to the Hafren stream after crossing the catchment. Chloride spectra of rainfall (dotted lines) resemble white noise; those of streamflow (solid lines) resemble $1 / \mathrm{f}$ noise, with spectral power increasing proportionally to wavelength across the entire range of scales (data measured daily for 3 years, and weekly for 14 years). Bottom: Response of streamflow concentrations to a delta-function pulse input of contaminants. Because of the long-tailed nature in comparison to conventional models, contaminant concentrations are sustained substantially for much longer time spans. The logarithmic concentration scale emphasises the persistence of low-level contamination of the $\Gamma$-fit $\propto t^{\alpha-1} e^{-t / \tau}$ ( $\alpha \simeq 0.5 ; \tau \simeq 1.9$ years is close to the edge of the data window such that essentially all data follow the power-law $t^{-1 / 2}$ ) used in the original work [Kirchner et al (2000)]. Inset depicts $\delta$-function contaminant input.

to exhibit anomalous tracer dispersion [Berkowitz et al (2000)], a problem still lacking a deeper understanding. In a similar manner, on-bed particle diffusion in gravel bed flows was recently shown to exhibit different transport regimes, ranging from ballistic to subdiffusion [Nikora et al (2002)].

\subsection{Biological systems}

Within a single biological cell, the motion of microspheres was found to have a transient superdiffusive behaviour with $\alpha=3 / 2$ (motor-driven motion), an exponent suspiciously close to the motion in random velocity fields [Matheron and de Marsily (1980), 

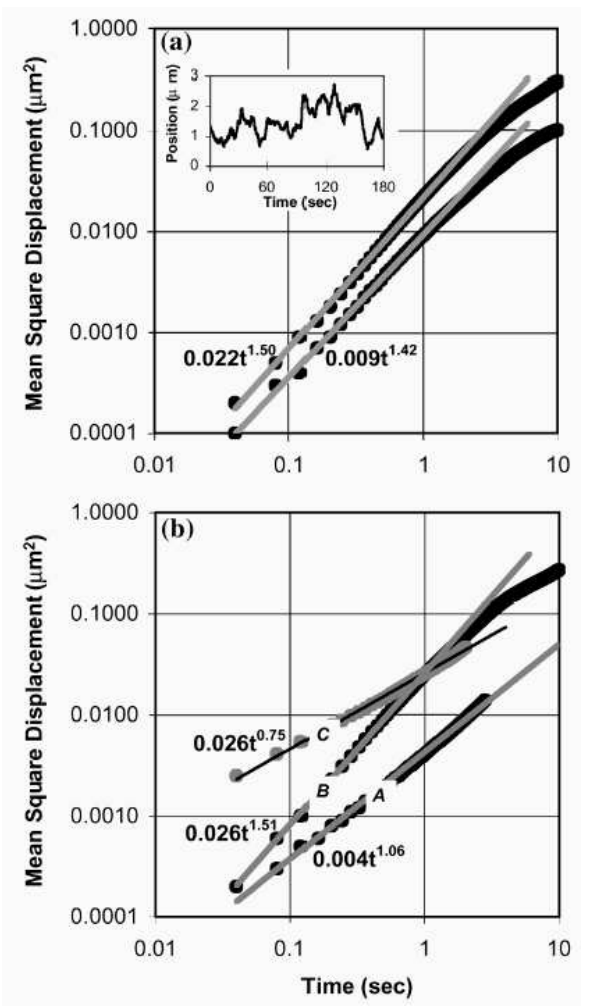

Figure 2. Mean squared displacement of engulfed microspheres in the cytoskeleton of a living cell. Active, motor-driven transport with exponent $3 / 2$ turns over to subdiffusion with exponent $3 / 4$ (occasionally normal diffusion) [Caspi et al (2000)].

Zumofen et al (1990]. $\dagger$ This active superdiffusion is followed by a subdiffusive (in some instances also normal diffusive) scaling [Caspi et al (2000), Caspi et al (2002), Caspi et al (2001)]. Some typical experimental results are depicted in figure 2. Similar subdiffusive behaviour in cells is known from E. coli cell membrane inclusions [Tolic-Nørrelykke et al (2003)]. We note that such presumably cytoskeleton-mediated anomalous diffusion patters are consistent with findings from diffusion assays of microspheres in polymer networks [Amblard et al (1996)], and time anomalies are also known from fluorescence video-microscopy assays [LeGoff et al (2002)], and microrheology experiments on semiflexible polymers [Wong et al (2003), Tseng and Wirtz (2002)], and from regular polymer melts [Fischer et al (1996), Kimmich (1997)]. The subdiffusive phenomenon may in fact be related to caging-caused subdiffusion [Weeks and Weitz (2002), Weeks et al (2000)].

In proteins, a detailed analysis based on Fourier transform infrared spectroscopy data [Iben et al (1989), Austin et al (1974), Austin et al (1975)] demonstrated that ligand rebinding to myoglobin follows an asymptotic power-law

$\dagger$ We note that anomalous diffusion-assisted ratchet transport was studied to some detail in references [Bao (2003), Bao and Zhuo (2003)], compare the fractional generalisation of the Kramers problem, which was originally formulated in reference [Metzler and Klafter (2000e)], see also [So and Liu (2004)]. 
decay. Data analysis showed that the entire measured rebinding curve follows fractional dynamics with Vogel-Fulcher type temperature activation [Glöckle and Nonnenmacher (1995)]. Measurements of single ion gating channels using the patch clamp technique show logarithmic oscillations around a powerlaw trend [Blatz and Magleby (1986)], which was demonstrated to correspond to a power-law distribution of characteristic times and amplitudes of individual exponential relaxation contributions [Nonnenmacher and Nonnenmacher (1989)]. Similarly, the passage of a single bio-oligo- or macromolecule through a membrane pore [Meller (2003)] was recently shown to have a priori unexpected long-time contributions [Bates et al (2003), Metzler and Klafter (2003), Flomenbom and Klafter (2003), Flomenbom and Klafter (2004)]. While for short chains anomalous time behaviour is most likely caused by chain-pore interactions (sticking) [Bates et al (2003)], in the case of long chains the anomalous nature follows a forteriori from the polymer relaxation time [Chuang et al (2002)]. We note that long passage times were also found in fluorescence microscopy single molecule assays of DNA uptake into the cell nucleus [Salman et al (2001)]. Compelling evidence for broad time scale distributions in protein conformational dynamics was reported recently, and modelled on the basis of the fractional Fokker-Planck equation [Yang et al (2003), Yang and Xie (2002)]. In figure 3, we reproduce the fluorescence autocorrelation function fitted by various functions, showing the superior quality of the anomalous diffusion model, as well as a reconstruction of the energy landscape of the protein conformation, see 3 for details.

In a double-stranded DNA heteropolymer made up of the nucleotides (bases) $\mathrm{A}$ (denine), $\mathrm{G}$ (uanine), $\mathrm{C}$ (ytosine), and $\mathrm{T}$ (hymine), the entropy-carrying, flexible single-stranded bubbles, which open up due to thermal fluctuations, are preferentially located in areas rich of the weaker AT bonds [Altan-Bonnet et al (2003), Hanke and Metzler (2003)]. On diffusion along the DNA backbone, the bubbles have to cross tighter GC-rich regions, an effect which was shown to produce subdiffusion [Hwa et al (2003)]. Similarly, the motion of DNA-binding proteins along DNA due to differences in the local structure is subdiffusive [Slutsky et al (2003)]. In contrast, the points at which a random walker on a polymer chain can jump to another chain segment, which is closeby in 3D space but distant in terms of the chemical coordinate, are distributed like an LF [Brockmann and Geisel (2003a), Sokolov et al (1997)], which may be contribute to fast target localisation of (regulatory) proteins along DNA [Berg et al (1981)], in particular, in respect to situations of overwhelming nonspecific binding [Bakk and Metzler (2004), Bakk and Metzler (2004a)], compare the on-DNA investigation in reference [Slutsky and Mirny (2004)]. We note that such dynamical features may be employed for DNA sequencing, which is in turn related to Lévy signatures [Scafetta et al (2002)]. A particle attached to a (biological) membrane and confined to an harmonic potential (as fulfilled to good approximation in an optical tweezers field) displays anomalous relaxation behaviour related to Mittag-Leffler functions [Granek and Klafter (2001)]. Also the boundary layer thickness around a membrane exhibits subdiffusive behaviour [Dworecki et al (2003), 

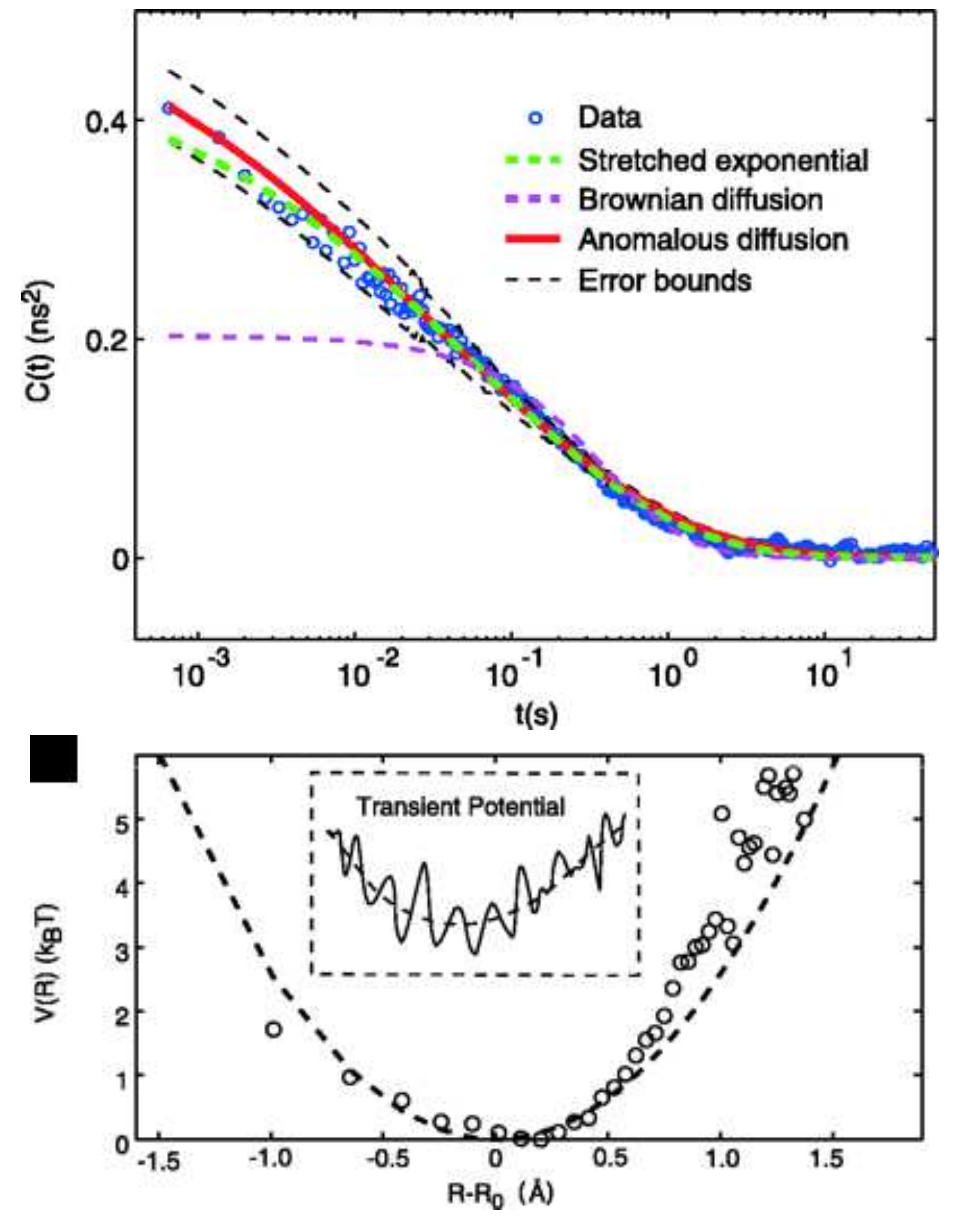

Figure 3. Top: Fit of the experimental autocorrelation function of fluorescence lifetime fluctuations by stretched exponential and anomalous diffusion models, in comparison to the rather bad fits by a Brownian diffusion model. Bottom: Potential of mean force calculated from measurements. The dashed line is a fit to a harmonic potential with variance of $0.19 \AA^{2}$. (Inset) A sketch of a rugged "transient" potential resulting from the short-time projection of $3 \mathrm{D}$ motions of the protein to the experimentally accessible coordinate [Yang et al (2003)].

Kosztołowicz and Dworecki (2003)].

On somewhat larger scales, NMR field gradient measurements of biological tissue [Köpf et al (1996)] could be shown to reveal anomalous diffusion behaviour in cancerous regions in both time and space resolution [Köpf et al (1998)]. Finally, the trajectories between turning or resting points of biological species within their habitats have been found to follow power-law statistics, such observations pertaining from bacteria [Shlesinger and Klafter (1990), Levandowsky et al (1997)] and plankton [Visser and Thygesen (2003)] over spidermonkeys [Ramos-Fernandez et al (2003)] and jackals [Atkinson et al (2002)], to the famed flight of an albatross [Viswanathan et al (1996), Viswanathan et al (1999)]. As an example, we show typical trajectories of spider-monkeys in the forest of the Mexican Yucatan peninsula in figure 4. In parts $\mathrm{c}$ and $\mathrm{d}$ of this figure, a zoom into 

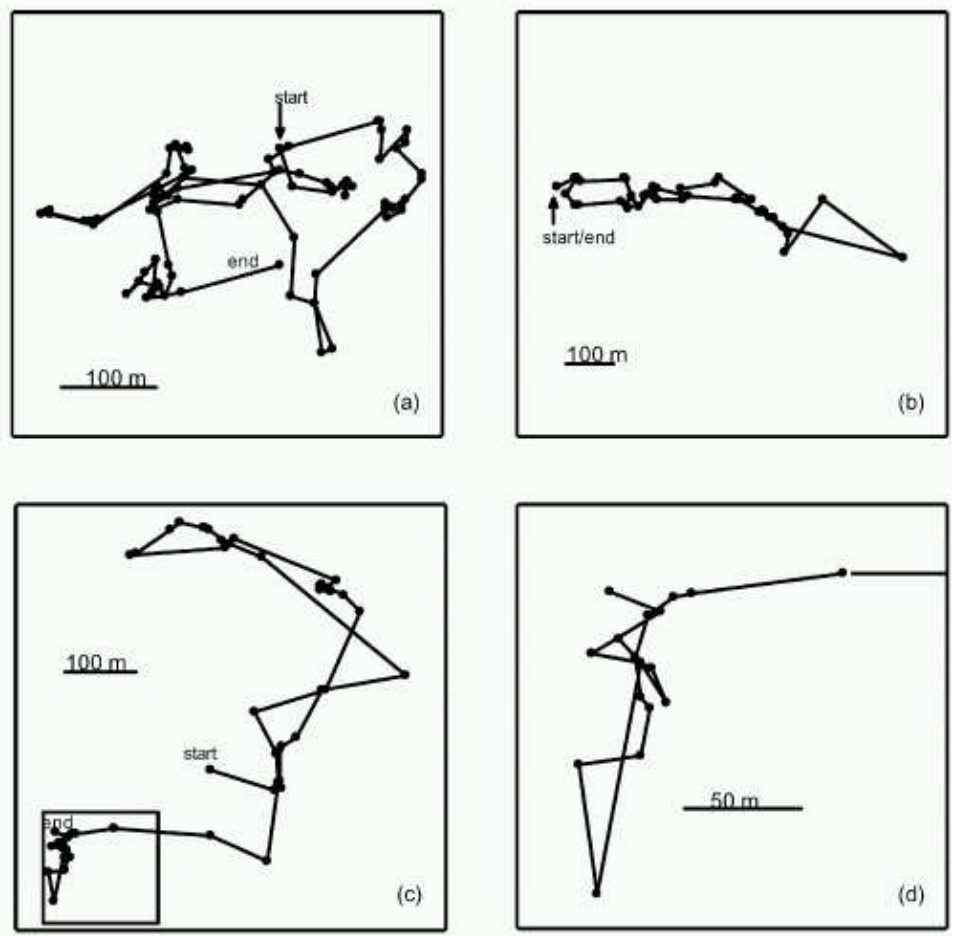

Figure 4. Daily trajectories of adult female $(a, b)$ and male (c) spider monkeys. In panel d, a zoom into the square of $\mathrm{c}$ is shown [Ramos-Fernandez et al (2003)].

the trajectory reveals a self-similar behaviour. Statistical analysis reveals a Lévy walk with an exponent in the mean squared displacement (1) of magnitude $\alpha \approx 1.7$ [Ramos-Fernandez et al (2003)].

\subsection{Small and large: Other system with anomalous dynamics}

In subrecoil laser cooling, 'velocity selective coherent population trapping' leads to a broadly distributed waiting time of particles close to zero momentum, the Lévy stable nature or which can in principle be measured. Moreover, its dynamical description exactly leads to a Riemann-Liouville fractional operator [Kondrashin et al (2002), Schaufler et al (1999), Schaufler et al (1999a), Bardou et al (2002)]. Similarly, anomalous diffusion occurs in optical lattices [Lutz (2003)]. Power-law statistics were observed for the histograms of on- and off-times in single quantum dots [Shimizu et al (2001)], see figure 5. Signatures of Lévy statistics were impressively documented in the study of the position of a single ion in a one-dimensional optical lattice, in which diverging fluctuations could be observed in the kinetic energy [Katori et al (1997)]. Lévy statistics has been identified in random single-molecule line shapes in glass-formers [Barkai et al (2000), Barkai et al (2003)]. Already in reference [Kenty (1932)] it was concluded that in radiation diffusion the rapidity of escape of resonance radiation from a gas leads to anomalous statistics according to which the fraction of emitted 


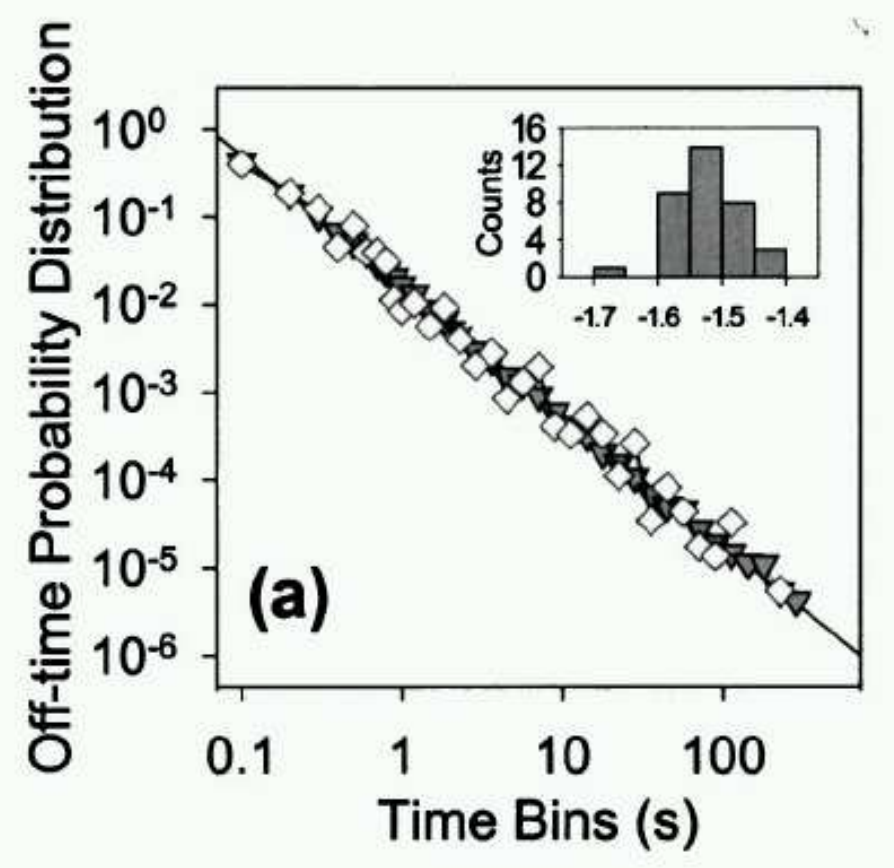

Figure 5. Off-time statistics of blinking (on-off cycles) quantum dots, exhibiting power-law statistics over several decades. For details see reference [Shimizu et al (2001)].

quanta traversing at least a given distance before absorption decays approximately linearly with the distance. Classical intermittency, expressed in terms of continuous time random walks and the LFFPE can be related to the quantum Anderson transition [García-García (2003)], and LF-signatures were proposed to underly the fracton excitations in certain 'unconventional' superconductors [Milovanov and Rasmussen (2002)].

Lévy-type random walks were recognised in the evolution of comets from the Oort cloud [Zhou et al (2002), Zhou and Sun (2001)], and anomalous diffusion was diagnosed in cosmic ray spectrum [Lagutin and Uchaikin (2003)]. It has recently been argued that the terrestrial temperature anomalies are inherited through a Lévy walk memory component from intermittent solar flares [Scafetta and West (2003)]. From radio signals received from distant pulsars, it has been proposed that the interstellar electron density fluctuations obeys Lévy statistics [Boldyrev and Gwinn (2003)]. A nonlinear fractional equation was proposed for the kinetic description of turbulent plasma and fields at the nonequilibrium stationary states of the magnetotail of Earth [Milovanov and Zelenyi (2002), Milovanov and Zelenyi (2001)]. Finally, ion motion along the direction normal to the magnetopause has been diagnosed to be of Lévy walk nature [Greco et al (2003)].

Anomalous diffusion was proposed to account for the hydrogen effect on the morphology of silicon electrodes under electrochemical conditions [Goldar et al (2001)], as well as in the context of nonlinear electrophoresis [Baskin and Zilberstein (2002)]. 
Fractional analysis tools were applied in the analysis of anomalous diffusion patterns found in amorphous electroactive materials [Bisquert et al (2003), Bisquert (2003)]. Anomalous diffusion of cations was found as the mechanism in the growth of surface Molybdenum-oxide patterns [Lugomer et al (2002)], and similarly the electron transfer kinetics in PEDOT $\ddagger$ films [Randriamahazaka et al (2002)] and atomic transport and chemical reaction processes in high- $k$ dielectric films [de Almeida and Baumvoll (2003)].

Fractional dynamics may underly the statistics of the joint velocity-position PDF of a single particle in turbulent flow [Friedrich (2003)]. A fractional generalisation of Richardson's law was proposed for the description of water transport in unsaturated soils [Pachepsky et al (2003)]. Lévy-type PDFs of particle velocities in softmode turbulence were studied in electroconvection [Tamura et al (2002)]. From a phenomenological point of view, LFs have been used to describe the dynamics observed in plasmas [Chechkin et al (2002a), Gonchar et al (2003), Bakunin (2003)], or in molecular collisions [Carati et al (2003)]. Stochastic collision models and their natural relation to Lévy velocity laws are discussed in reference [Barkai (2003)]. A fractional diffusion approach to the force distribution in static granular media was brought forth recently [Vargas et al (2003)]. Anomalous diffusion properties of heat channels has been investigated in [Denisov et al (2003), Reigada et al (2002)].§ Surface growth under certain circumstances requires a generalisation of the classical KardarParisi-Zhang model. Recent discussion involves a space-fractional KPZ-equation [Katzav (2003), Mann and Woyczynski (2001)], compare the discussion of anomalous surface diffusion in [Naumovets and Zhang (2002), Vega et al (2002)], fractal growth [Leith (2003)], and of travelling fronts in the presence of non-Markovian processes [Feodotov and Mendez (2000)]. In fact, it seems to turn out that the KPZ equation itself for a single growth column corresponds to a specific subordination to the regular Langevin equation [Falla et al (2004)].

Dielectric susceptibilities in glassy system are of strong non-Debye form (compare [Déjardin (2003), Metzler and Klafter (2002)]) and can in some systems be studied over some 15 decades in frequency $^{+}$[Hilfer (2002), Hilfer (2002a), Schneider et al (1999), Lunkenheimer and Loidl (2002)], and by NMR both subdiffusion in percolation clusters and Lévy walks in porous media have been verified [Kimmich (2002), Stapf (2002), Stapf (1995)]. In reference [Klemm et al (2002)], the PDF of fractional diffusion is shown to account for the measured, projected self-diffusion profiles on a fractal percolation structure. $1 / f$-noise and correlated intermittent behaviour was reported from molecular dynamics simulations of water-freezing [Matsumoto et al (2002)].

In economical contexts, it has been revealed that Lévy statistics are present in the distribution of trades [Mandelbrot (1963), Mandelbrot (1966), Mandelbrot (1967a),

$\ddagger$ Poly-3,4-Ethylenedioxythiophene

$\S$ Also compare to reference [Li and Wang (2003)] and the Comment on that paper [Metzler and Sokolov (2004)].

+ Similar to master curves from rubbery systems [Glöckle and Nonnenmacher (1991), Metzler et al (1995)]. 


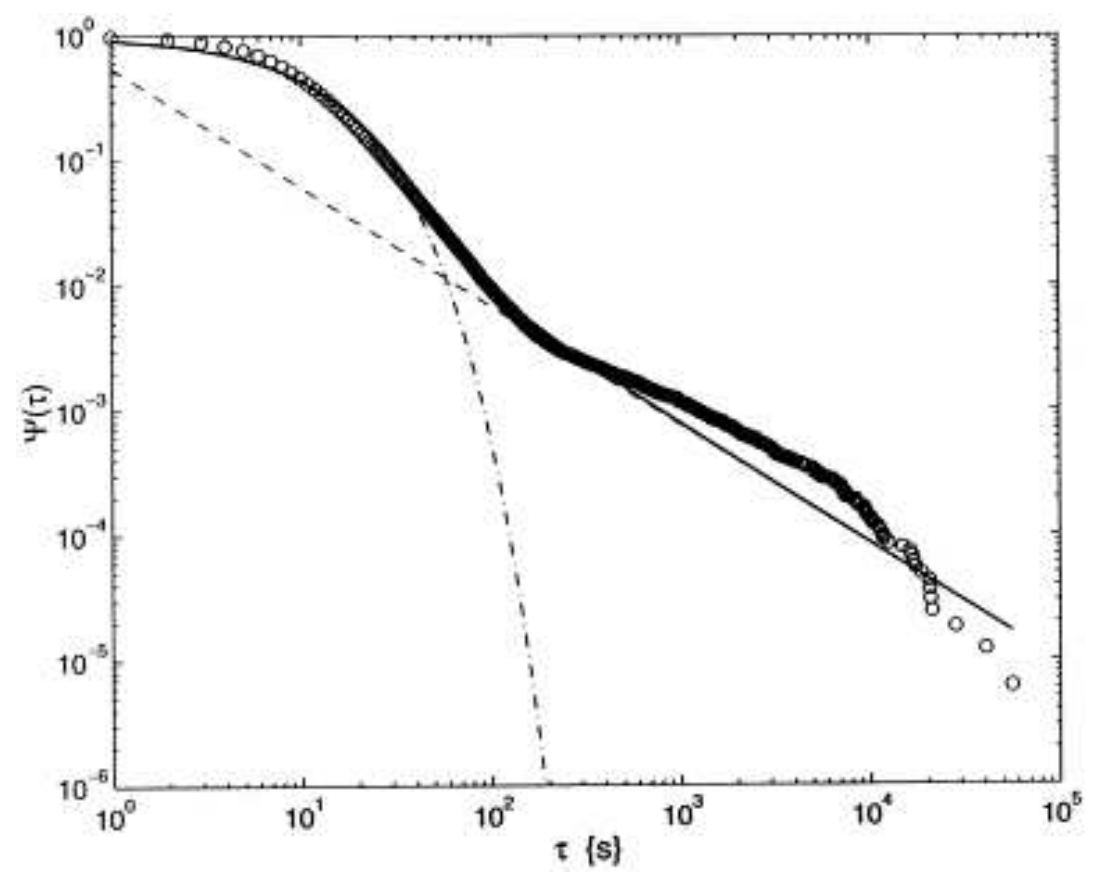

Figure 6. Survival probability for BUND futures with from September 1997. The Mittag-Leffler function (full line) is compared with a stretched exponential (dasheddotted) and a power-law (dashed) [Mainardi et al (2000)].

Mantegna and Stanley (1996), Mantegna and Stanley (2000), Bouchaud and Potters (2000)]. Similarly, it was shown that 'fat tails' appear in the return of the value of a given asset to a fixed level [Jensen et al (2003), Simonsen et al (2002)]. For the waiting time between two transactions power-law statistics were observed [Kim and Yoon (2003), Raberto et al (2002), Scalas et al (2000), Mainardi et al (2000)]. Figure 6 shows a Mittag-Leffler fit to the BUND futures traded in September 1997, in which the initial $\approx 2.5$ decades in time are nicely fitted by the Mittag-Leffler function (which has a point of inflection for index larger $1 / 2$, afterwards the data appear to oscillate around the Mittag-Leffler trend 6.

Finally, we note that ageing in glasses and other disordered systems [Monthus and Bouchaud (1996), Rinn et al (2000), Pottier (2003)] as well as in dynamical systems [Barkai and Cheng (2003), Barkai (2003a)] involve power-law non-locality in time, compare the discussion in [Sokolov et al (2001)] and [Allegrini et al (2003)].

\section{Subdiffusive processes}

Subdiffusive dynamics is characterised by strong memory effects on the (fluctuationaveraged) level of the PDF $P(x, t)$, i.e., unlike in a Markov process the now-state of the system depends on the entire history from its preparation [Barkai (2001), Hughes (1995), Metzler and Klafter (2000)]. This contrasts generalised Langevin equations, whose fluctuation average produces equations for $P(x, t)$, which carry 
time-dependent transport coefficients but are local in time [Wang et al (1994), Wang and Tokuyama (1999), Bazzani et al (2003)]. Subdiffusion is classically described in terms of the CTRW (see appendix) with a long-tailed waiting time PDF of the asymptotic form

$$
\psi(t) \sim \tau^{\alpha} / t^{1+\alpha}, \quad 0<\alpha<1
$$

for $t \gg \tau$. In fact, subdiffusive processes are directly subordinated to their analogous Markovian system through a waiting time PDF $\psi(t)$ of the above form. Such waiting times are distinguished by the divergence of the characteristic waiting time, $\mathfrak{T}=\int_{0}^{\infty} \psi(t) t d t=\infty$, and they reflect the existence of deep traps, which subsequently immobilise the diffusing particle. The seminal case study for such processes are amorphous semiconductors [Pfister and Scher (1977), Pfister and Scher (1978), Scher and Montroll (1975)].

\subsection{Fractional diffusion equation}

From expression (2), we can immediately obtain the equation for $P(x, t)$ in the force-free case. To this end, we combine the long-tailed $\psi(t)$ with a short-ranged jump length PDF $\lambda(x)$ and the known expression (A.1) for the PDF $P(x, t)$ in the continuous time random walk model (see [Metzler and Klafter (2000)] and the appendix). With the asymptotic behaviour

$$
\psi(u) \equiv \mathscr{L}\{\psi(t) ; u\}=\int_{0}^{\infty} \psi(t) \exp (-u t) d t \sim 1-(u \tau)^{\alpha}
$$

of the Laplace transform $\mathscr{L}\{\psi(t) ; u\}$ of $\psi(t)$, and the analogous expansion of a typical, short-ranged jump length $\mathrm{PDF}, \lambda(k) \sim 1-\sigma k^{2}(k \rightarrow 0)$ for the Fourier transform of $\lambda(x)$, we obtain

$$
P(k, u) \simeq \frac{1 / u}{1+u^{-\alpha} K_{\alpha} k^{2}},
$$

where we identified the anomalous diffusion constant as $K_{\alpha} \equiv \sigma / \tau^{\alpha}$. By the symbol $\simeq$, we indicate that the result for $P(k, u)$ is based on expansions for $\psi(u)$ and $\lambda(k)$. However, similar to the limit in going from the master equation to the continuum limit, we can choose $\tau$ and $\sigma$ small enough (keeping $K_{\alpha}$ finite), such that $P(k, u)$ essentially covers the entire time-space range. In this sense, we are going to drop the $\simeq$-sign in the following.

In the Brownian limit $\alpha=1$, expression (4) after multiplication by the denominator leads to the standard diffusion equation $\partial P(x, t) / \partial t=K_{1} \partial^{2} P(x, t) / \partial x^{2}$, making use of the integral theorem $\mathscr{L}\{d f(t) / d t\}=u^{-1} f(u)$ and the differentiation theorem $f\left\{d^{2} g(x) / d x^{2}\right\}=-k^{2} g(k)$ of the Laplace and Fourier transformations, respectively [Wolf (1979)]. By partial differentiation of the obtained integral equation, the diffusion equation yields. For the subdiffusive case $0<\alpha<1$, in contrast, a term of the form $u^{-\alpha} f(u)$ occurs. Its Laplace inversion is indeed feasible, due to the property

$$
\mathscr{L}\left\{{ }_{0} D_{t}^{-\alpha} f(t)\right\} \equiv \int_{0}^{\infty} d t e_{0}^{-u t} D_{t}^{-\alpha} f(t)=u^{-\alpha} f(u)
$$


of the Riemann-Liouville fractional integral:

$$
{ }_{0} D_{t}^{-\alpha} f(t) \equiv \frac{1}{\Gamma(\alpha)} \int_{0}^{t} d t^{\prime} \frac{f\left(t^{\prime}\right)}{\left(t-t^{\prime}\right)^{1-\alpha}},
$$

defined for any sufficiently well-behaved function $f(t)$ [Miller and Ross (1993), Oldham and Spanier (1974), Podlubny (1998), Samko et al (1993)]. Thus, from equation (4) we obtain the fractional diffusion equation in the so-called integral form [Balakrishnan (1985), Schneider and Wyss (1989)],

$$
P(x, t)-P_{0}(x)={ }_{0} D_{t}^{-\alpha} K_{\alpha} \frac{\partial^{2}}{\partial x^{2}} P(x, t),
$$

where we have written a general initial condition $P_{0}(x)$ instead of the $\delta$-condition $P_{0}(x)=\delta(x)$ corresponding to (4). By partial differentiation and with the RiemannLiouville fractional differential operator

$$
{ }_{0} D_{t}^{1-\alpha} \equiv \frac{\partial}{\partial t}{ }_{0} D_{t}^{-\alpha}
$$

we arrive at the usual form of the fractional diffusion equation

$$
\frac{\partial}{\partial t} P(x, t)={ }_{0} D_{t}^{1-\alpha} K_{\alpha} \frac{\partial^{2}}{\partial x^{2}} P(x, t) .
$$

The solution of this equation can be obtained in closed form in terms of the Fox $H$-function [Hilfer (1995), Metzler and Klafter (2000), Metzler and Klafter (2000c), Schneider and Wyss (1989)]. Moreover, due to the definition of this $H$-function as a Mellin-Barnes integral, the spectral functions of $P(x, t)$ such as $P(k, t), P(k, \omega)$ etc. can be obtained in closed form, as well [Metzler and Nonnenmacher (1997)]. As we are mainly interested in processes in the presence of an external force field, we only stop to note that the asymptotic behaviour of the propagator $P(x, t)$ of the fractional diffusion equation (9) corresponds to the stretched Gaussian shape listed in table 1 . The PDF of such a subdiffusive diffusion process, that is, has a softer decay than that of normal diffusion. In return, the Fourier transform, $P(k, t)$, is of Lévy stable character [Metzler and Klafter (2000), Metzler and Nonnenmacher (1997)].

We should point out that it is important to keep track of the initial condition in the fractional diffusion equation (9). Thus, one can by the standard property ${ }_{0} D_{t}^{\alpha} 1=t^{-\alpha} / \Gamma(1-\alpha)$ of the Riemann-Liouville operator retrieve the equivalent equation to (9) in the form

$$
{ }_{0} D_{t}^{\alpha} P(x, t)-\frac{P_{0}(x)}{\Gamma(1-\alpha)} t^{-\alpha}=K_{\alpha} \frac{\partial^{2}}{\partial x^{2}} P(x, t) .
$$

Neglecting the initial condition would lead to a wrong equation, as can easily be seen by calculating the average on both sides.

We can now also compare the memory form of equation (9) with the dynamical equation for FBM [Lutz (2001a)],

$$
\frac{\partial}{\partial t} P_{\mathrm{FBM}}(x, t)=\alpha K_{\alpha} t^{\alpha-1} \frac{\partial^{2}}{\partial x^{2}} P_{\mathrm{FBM}}(x, t)
$$


which is perfectly local in time. This equation for FBM can be derived from the forcefree GLE [Lutz (2001a)]

$$
m \frac{\mathrm{d}^{2}}{\mathrm{~d} t^{2}} x(t)+m \eta_{\alpha 0} D_{t}^{\alpha} x(t)=\Gamma(t)
$$

where $\Gamma(t)$ is Gaussian random noise with variance $\langle\Gamma(t) \Gamma(0)\rangle \propto t^{-\alpha}$. This GLE gives then also rise to Mittag-Leffler type correlation functions, see reference [Lutz (2001a)] for more details.

\subsection{Fractional Fokker-Planck equation}

The incorporation of an external force can be achieved by choosing an explicitly space-dependent form for the jump length PDF, such that one can account for the spatial inhomogeneity due to a general force field $F(x)=-d V(x) / d x$. From this model one infers the fractional Fokker-Planck equation, as detailed in references [Barkai et al (2000a), Metzler et al (1999a)]. However, here we prefer to present here a somewhat more fundamental derivation leading to a fractional Fokker-Planck equation in phase space.

To this avail, we come back to the idea of interpreting the subdiffusion process as a subordination to a Brownian process, in the following sense. This subordination is intuitively described by the adjunct microscopic multiple trapping process. As detailed in [Metzler and Klafter (2000a), Metzler and Klafter (2000b), Metzler (2000)], based on the continuous time version of the Chapman-Kolmogorov equation, the motion events in this multiple trapping picture are based on a regular, Markovian random walk process, governed through the Langevin equation [Langevin (1908), Chandrasekhar (1943), van Kampen (1981)]

$$
m \frac{d^{2} x}{d t^{2}}=-\eta m \frac{d x}{d t}+F(x)+m \Gamma(t)
$$

where $\Gamma(t)$ denotes a $\delta$-correlated Gaussian noise, i.e., $\overline{\Gamma(t) \Gamma\left(t^{\prime}\right)}=2 K \delta\left(t-t^{\prime}\right)$ and the noise characteristic function is $\varphi(k)=\int_{-\infty}^{\infty} \exp (i k \Gamma) p(\Gamma) d \Gamma=\exp \left(-K k^{2}\right)$. ${ }^{*}$ Each motion event governed through the Langevin equation (13) is supposed to last an average time span $\tau^{*}$, and each such single motion event is interrupted by immobilisation (trapping) of a duration governed by the broad waiting time PDF (2). Averaging over many such motion-immobilisation events, one obtains the multiple trapping scenario leading to subdiffusion in the external field $F(x)$ [Metzler and Klafter (2000a), Metzler and Klafter (2000b), Metzler (2000)], which may be viewed as a direct consequence of the generalised central limit theorem [Gnedenko and Kolmogorov (1954), Lévy (1954)]. In the Markov limit, the waiting time PDF $\psi(t)$ possesses a finite characteristic waiting time $\mathfrak{T}$, and may for instance be given by the exponential $\psi(t)=\tau^{-1} \exp (-t / \tau)$, or a sharp distribution such as $\psi(t)=\delta(t-\tau)$. Note that in Laplace space, $\psi(u) \simeq 1-(u \tau)^{\alpha}(u \tau \ll 1)$, both subdiffusive $(0<\alpha<1)$ and Markov $(\alpha=1)$ limits appear unified.

\footnotetext{
* We denote fluctuation averages by an overline, - , and coordinate averages by angular brackets, $\langle\cdot\rangle$.
} 
In phase space spanned by velocity $v$ and position $x$, a test particle governed by the above multiple trapping process is described in terms of the fractional Klein-Kramers equation (FKKE)

$$
\begin{aligned}
\frac{\partial P}{\partial t}={ }_{0} D_{t}^{1-\alpha} & \left(-v^{*} \frac{\partial}{\partial x}+\frac{\partial}{\partial v}\left(\eta^{*} v-\frac{F^{*}(x)}{m}\right)\right. \\
& \left.+\frac{\eta^{*} k_{B} T}{m} \frac{\partial^{2}}{\partial v^{2}}\right) P(x, v, t)
\end{aligned}
$$

with the abbreviations $v^{*} \equiv v \tau^{*} / \tau^{\alpha}, \eta^{*} \equiv \eta \tau^{*} / \tau^{\alpha}$, and $F^{*}(x) \equiv F(x) \tau^{*} / \tau^{\alpha}$ [Metzler and Klafter (2000a), Metzler and Klafter (2000b), Metzler (2000)]. Note that the Stokes operator $\left(\frac{\partial}{\partial t}+v \frac{\partial}{\partial x}\right)$ from the standard Klein-Kramers equation [Chandrasekhar (1943)] is replaced by the operator $\left(\frac{\partial}{\partial t}+{ }_{0} D_{t}^{1-\alpha} v^{*} \frac{\partial}{\partial x}\right)$ which shows the non-local drift response due to trapping.

For both the Langevin equation (13) and the FKKE (14) one can consider the under- (velocity equilibration) and overdamped (large friction constant) limits. The former limit corresponds to the fractional version of the Rayleigh equation [van Kampen (1981)],

$$
\frac{\partial P}{\partial t}={ }_{0} D_{t}^{1-\alpha} \eta^{*}\left(\frac{\partial}{\partial v} v+\frac{k_{B} T}{m} \frac{\partial^{2}}{\partial v^{2}}\right) P(v, t) .
$$

in the force-free limit [Metzler and Klafter (2000a), Metzler and Klafter (2000b)]. This is the subdiffusive generalisation of the Ornstein-Uhlenbeck process, see also below. Conversely, in the overdamped case, the FKKE (14) corresponds in position space to the fractional Fokker-Planck equation (FFPE) [Metzler et al (1999), Metzler et al (1999a), Metzler and Klafter (2000a), Metzler and Klafter (2000b)]

$$
\frac{\partial P}{\partial t}={ }_{0} D_{t}^{1-\alpha}\left(-\frac{\partial}{\partial x} \frac{F(x)}{m \eta_{\alpha}}+K_{\alpha} \frac{\partial^{2}}{\partial x^{2}}\right) P(x, t) .
$$

We note that all fractional equations, (14), (15), and (16) reduce to their Markov counterparts in the limit $\alpha \rightarrow 1$, which can be seen from both the reduction of the multiple trapping process to the regular random walk, and the properties of the Riemann-Liouville fractional operator. We also note that in the general case of $0<\alpha<1$, initial conditions are strongly persistent due to the slow decay of the sticking probability of not moving $\phi(t)=1-\int_{0}^{t} \psi(t) d t$, i.e., one observes characteristic cusps at the location of a sharp initial PDF, e.g., $P(x, 0)=\delta\left(x-x_{0}\right)$, compare figure 8, and [Metzler and Klafter (2000)] for more details. The fractional equations following from the multiple trapping model with broad waiting time PDF give rise to a generalised Einstein-Stokes relation $K_{\alpha}=k_{B} T /\left(m \eta_{\alpha}\right)$ and fulfil linear response in the presence of a constant field $F_{0}$ [Metzler et al (1999), Metzler and Klafter (2000)]:

$$
\langle x(t)\rangle_{F_{0}}=\frac{k_{B} T}{2}\left\langle x^{2}(t)\right\rangle_{F=0} .
$$

The calculation of moments from fractional equations of the FFPE (16) kind can be straightforwardly obtained by multiplying the dynamical equation with the moment variable and integration over the coordinate, e.g., calculating $\int x^{m} \cdot d x$ where $\cdot$ acts on 


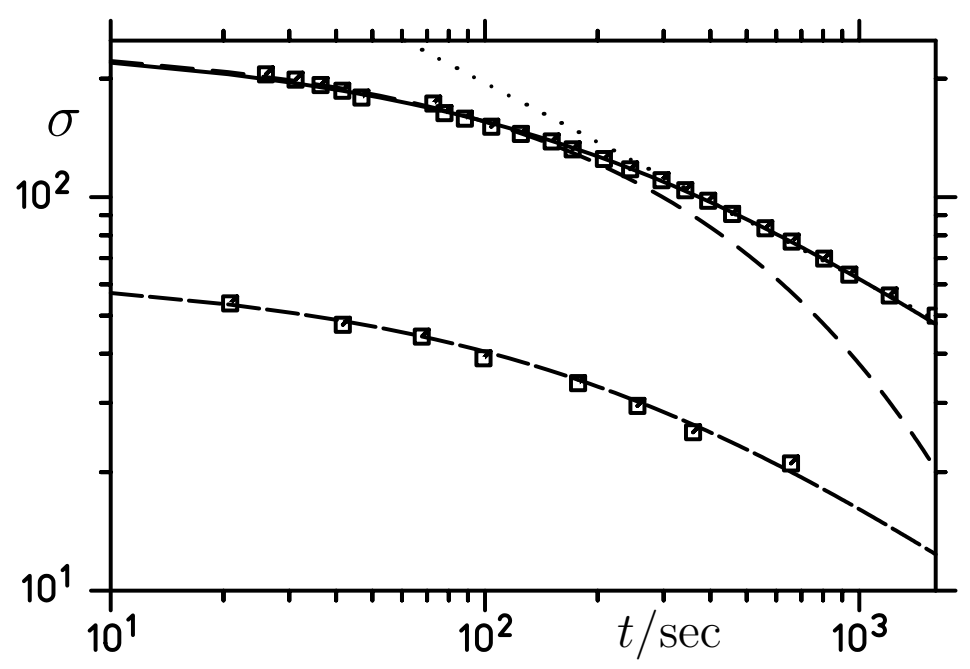

Figure 7. Interpolative nature of the Mittag-Leffler function, in an example from stress relaxation at constant strain (the image shows two different initial conditions). In the upper curve, we compare the Mittag-Leffler function (full line), with the initial stretched exponential and the terminal inverse power-law behaviours. (From Ref. [Nonnenmacher (1991)].)

the dynamical equation [Metzler and Klafter (2000)]. More-point correlation functions are somewhat more difficult to obtain due to the strongly non-local character in time. Three-point correlation functions have recently been obtained on the basis of the FFPE by introducing the associated backward equation [Barsegov and Mukamel (2004)].

Fractional equations of the above linear, uncoupled kind can be solved by the method of separation of variables. Thus, for instance, the FFPE (16) can be separated through the ansatz $P(x, t)=X(x) T(t)$ to produce a spatial eigenequation, which has the same structure as its Markov analogue, and a temporal eigenequation,

$$
\frac{d T_{n}(t)}{d t}=-\lambda_{n 0} D_{t}^{1-\alpha} T_{n}(t)
$$

for a given eigenvalue $\lambda_{n}$ [Metzler et al (1999), Metzler and Klafter (2000)]. Its solution yields in terms of the Mittag-Leffler function [Mittag-Leffler (1903), Mittag-Leffler (1904), Mittag-Leffler (1905), Erdélyi (1954)]

$$
E_{\alpha}\left(-\lambda_{n} t^{\alpha}\right) \equiv \sum_{j=0}^{\infty} \frac{\left(-\lambda_{n} t^{\alpha}\right)^{j}}{\Gamma(1+\alpha j)} \sim \begin{cases}\exp \left(-\frac{\lambda_{n} t^{\alpha}}{\Gamma(1+\alpha)}\right), & t \ll \lambda^{1 / \alpha} \\ \left(\lambda_{n} t^{\alpha} \Gamma(1-\alpha)\right)^{-1}, & t \gg \lambda^{1 / \alpha}\end{cases}
$$

where we also indicated the interpolation property of the Mittag-Leffler function, connecting between an initial stretched exponential (KWW) pattern and a terminal inverse power-law behaviour [Metzler and Klafter (2000), Glöckle and Nonnenmacher (1991), Glöckle and Nonnenmacher (1994)], compare figure 7. As for a non-trivial external field $F(x)$, the lowest eigenvalue vanishes, $\lambda_{1}=0$, and thus $0<\lambda_{2}<\ldots$, the $\operatorname{PDF} P(x, t)$ relaxes toward the equilibrium solution given by the lowest eigenvalue $\lambda_{1}$ which is identical to the Boltzmann solution and fulfils the stationarity condition $\partial P(x, t) / \partial t=0$ 
[Metzler et al (1999), Metzler and Klafter (2000)]. Finally, we note that due to the Laplace space scaling relation [Metzler et al (1999), Metzler and Klafter (2000)]

$$
P(x, u)=\frac{1}{u} \frac{\eta_{\alpha} u^{\alpha}}{\eta_{1}} P_{M}\left(x, \frac{\eta_{\alpha} u^{\alpha}}{\eta_{1}}\right),
$$

for the same initial condition $P_{0}(x)$ between the solution of the FFPE $(16), P(x, t)$, and its Markov counterpart $P_{M}(x, t)$, there exists the generalised Laplace transformation [Barkai and Silbey (2000)]

$$
P(x, t)=\int_{0}^{\infty} d s E_{\alpha}(s, t) P_{M}(x, s)
$$

which corresponds to a generalised Laplace transformation from $t$ to $\frac{\eta_{\alpha}}{\eta_{1}} u^{\alpha}$. The kernel $E_{\alpha}(s, t)$ is defined in terms of the inverse Laplace transformation $E_{\alpha}(s, t)=$ $\mathscr{L}^{-1}\left\{\frac{\eta_{\alpha}}{\eta_{1} u^{1-\alpha}} \exp \left(-\frac{\eta_{\alpha}}{\eta_{1}} u^{\alpha} s\right)\right\}$, the result being the modified one-sided Lévy distribution 
$L_{1-\alpha / 2}^{+} \|$

$$
E_{\alpha}(s, t)=\frac{t}{(1-\alpha / 2) s} L_{1-\alpha / 2}^{+}\left(\frac{t}{\left(s^{*}\right)^{1 /(1-\alpha / 2)}}\right), \quad s^{*} \equiv \eta_{\alpha} s / \eta_{1},
$$

which is everywhere positive definite. Consequently, the transformation (21) guarantees the existence and positivity of $P_{\alpha}(x, t)$ if (and only if) the Brownian counterpart, $P_{M}(x, t)$, is a proper PDF. We note that the solution of certain classes of fractional equations are intimately related to the Fox $H$-function and related special functions [Mathai and Saxena (1978), Srivastava et al (1982), Saxena and Saigo (2001)]. It should be noted once more that fractional diffusion in the above defined Riemann-Liouville sense is fundamentally different \| In this review, we use symmetric Lévy-stable laws with characteristic function $\varphi(z)=$ $\int_{-\infty}^{\infty} e^{i k x-\sigma^{\mu}|k|^{\mu}} d k /(2 \pi)$. The definition of Lévy laws is more general. Thus, one-sided Lévy-stable laws exist, which are defined only on the positive semidefinite axis, i.e., in our case on the causal time-line $t \geq 0$. In terms of the general characteristic function $\varphi$ of a Lévt-stable law, defined through

$$
\log \varphi(z)=-|z|^{\alpha} \exp \left(\mathrm{i} \frac{\pi \beta}{2} \operatorname{sign}(z)\right)
$$

the one-sided laws exist for $0<\alpha<1$ and $\beta=-\alpha$. For instance, the one-sided stable law for $\alpha=1 / 2$ and $\beta=-1 / 2$ is given by

$$
f_{1 / 2,-1 / 2}=\frac{1}{2 \sqrt{\pi}} x^{-3 / 2} \exp (-1 /[4 x]),
$$

where, in general, we have

$$
f_{\alpha, \beta} \equiv \frac{1}{\pi} \operatorname{Re} \int_{0}^{\infty} \exp \left(-\mathrm{i} x z-z^{\alpha} \exp \left\{\mathrm{i} \frac{\pi \beta}{2}\right\}\right) .
$$

The parameter space of Lévy stable laws can be represented by the 'Takayasu diamond') [Takayasu (1990)]:

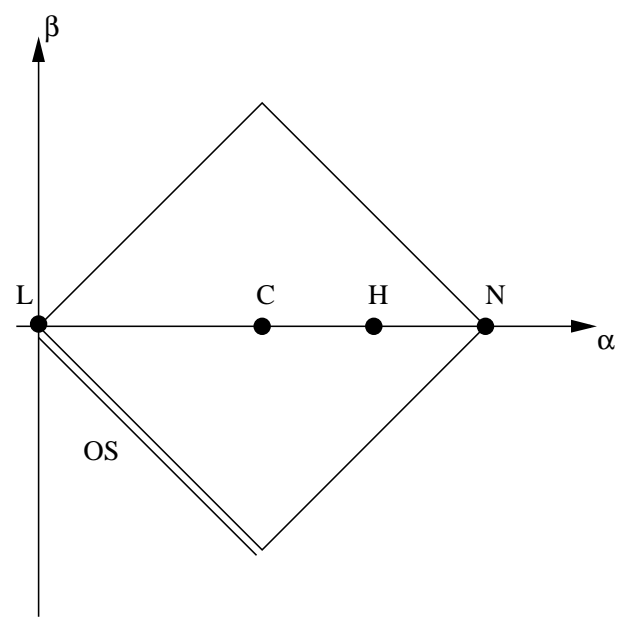

All pairs of indices inside and on the edge of the diamond shape refer to proper stable laws. The double line denotes one-sided stable laws (OS). The letters represent the normal or Gaussian law $(\mathrm{N})$, the Holtsmark distribution (H), and the Cauchy-Lorentz distribution (C) [Feller (1968), Gnedenko and Kolmogorov (1954), Lévy (1954), Takayasu (1990)].

We note that the connection of the fractional integral with stable distributions was recently investigated explicitly in reference [Stanislavsky (2004)]. 


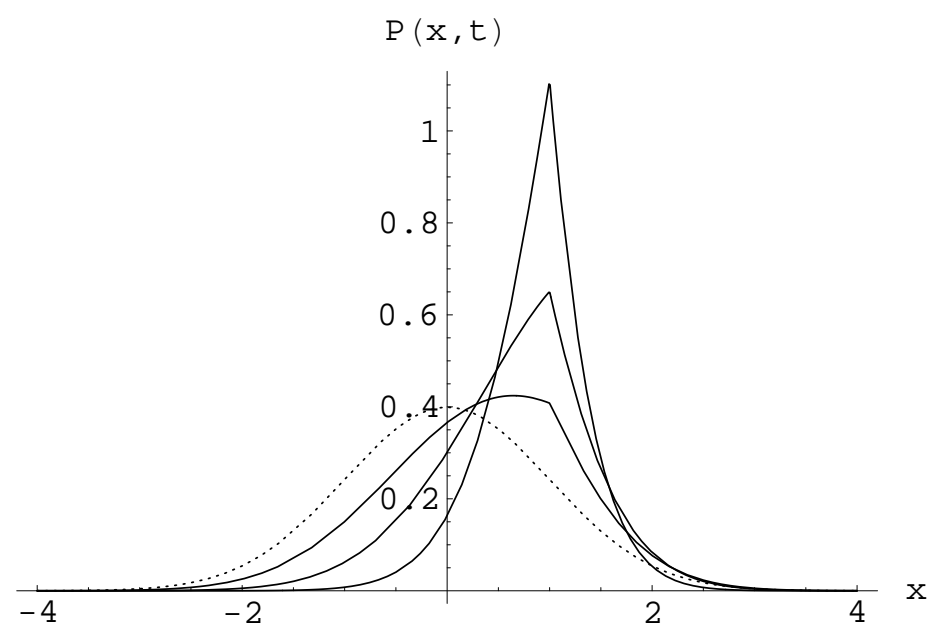

Figure 8. Time evolution of the PDF of the fractional Ornstein-Uhlenbeck process $(\alpha=1 / 2)$. The initial condition was chosen as $\delta(x-1)$. Note the strongly persistent cusp at the location of the initial peak. Dimensionless times: 0.02, 0.2, 2. The dashed line corresponds to the Boltzmann equilibrium.

from fractional Brownian motion [Mandelbrot and van Ness (1968), Lévy (1953), Lim and Muniandy (2002), Kolmogorov (1940)] and generalised Langevin equation approaches [Kubo et al (1985), Wang et al (1994), Wang and Tokuyama (1999)], compare table 1, as well as non-linear (fractional) Fokker-Planck equations [Borland (1998), Lenzi et al (2003a), Tsallis and Lenzi (2002)].

\subsection{The fractional Ornstein-Uhlenbeck process}

The Ornstein-Uhlenbeck process corresponds to the motion in an harmonic potential $V(x)=\frac{1}{2} m \omega^{2} x^{2}$ giving rise to the restoring force field $F(x)=-m \omega^{2} x$, i.e., to the dynamical equation

$$
\frac{\partial}{\partial t} P(x, t)=\left(\frac{\partial}{\partial x} \frac{\omega^{2} x}{\eta_{\alpha}}+K_{\alpha} \frac{\partial^{2}}{\partial x^{2}}\right) P(x, t) .
$$

From separation of variables, and the definition of the Hermite polynomials [Abramowitz and Stegun (1972)], one finds the series solution for the fractional FokkerPlanck equation with the Ornstein-Uhlenbeck potential [Metzler et al (1999)],

$$
\begin{aligned}
P(x, t)=\sqrt{\frac{m \omega^{2}}{2 \pi k_{B} T}} & \sum_{n=0}^{\infty} \frac{1}{2^{n} n !} E_{\alpha}\left(-\frac{n \omega^{2} t^{\alpha}}{\eta_{\alpha}}\right) H_{n}\left(\frac{\sqrt{m} \omega x_{0}}{\sqrt{2 k_{B} T}}\right) \\
& \times H_{n}\left(\frac{\sqrt{m} \omega x}{\sqrt{2 k_{B} T}}\right) \exp \left(-\frac{m \omega^{2} x^{2}}{2 k_{B} T}\right)
\end{aligned}
$$

plotted in figure 8. Individual position space modes follow the ordinary Hermite polynomials of increasing order, while their temporal relaxation is of Mittag-Leffler form, with decreasing internal time scale $\left(\eta_{\alpha} /\left[n \omega^{2}\right]\right)^{1 / \alpha}$. Numerically, the solution (24) 


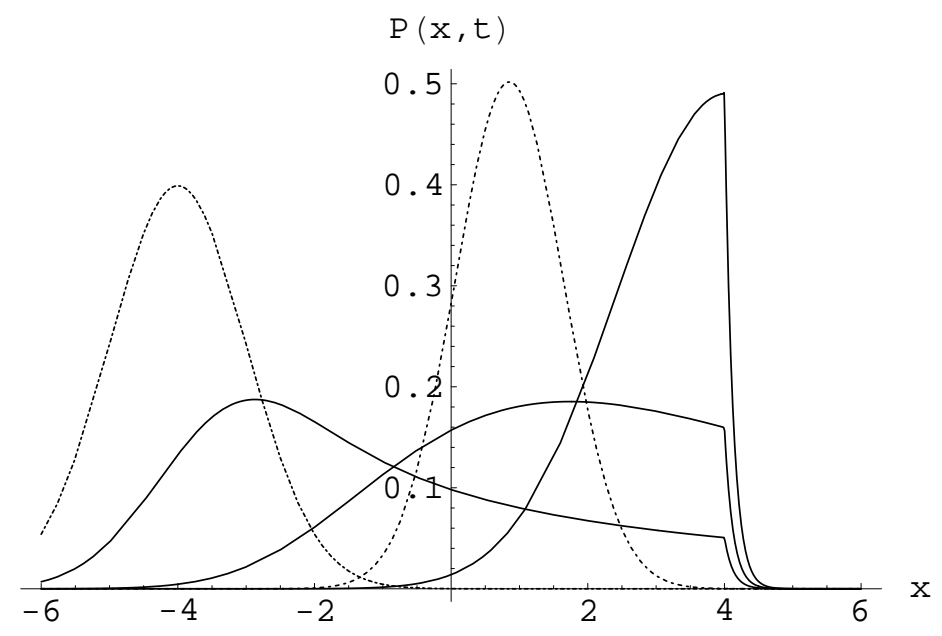

Figure 9. Time evolution of the PDF of the fractional Ornstein-Uhlenbeck process with superposed constant force of dimensionless strength $V=-4(\alpha=1 / 2)$. The initial condition was chosen as $\delta(x-4)$. Dimensionless times: $0.02,0.2,2$. The dashed lines corresponds to the Brownian solution at times 0.5 and 50 (in essence, the stationary state). Again, note the cusps due to the initial condition, causing a strongly asymmetric shape of the PDF in contrast to the Gaussian nature of the Brownian counterpart.

is somewhat cumbersome to treat. In order to plot the $\operatorname{PDF} P(x, t)$ in figure 8 , it is preferable to use the closed form solution (we use dimensionless variables)

$$
P(x, t)=\frac{1}{\sqrt{2 \pi\left(1-e^{-2 t}\right)}} \exp \left(-\frac{\left(x-x_{0} e^{-t}\right)^{2}}{2\left(1-e^{-2 t}\right)}\right)
$$

of the Brownian case, and the transformation (21) to construct the fractional analogue.

Figure 8 shows the distinct cusps at the position of the initial condition at $x_{0}=1$. The relaxation to the final Gaussian Boltzmann PDF can be seen from the sequence of three consecutive times. Only at stationarity, the cusp gives way to the smooth Gaussian shape of the Boltzmann equilibrium PDF. By adding an additional linear drift $V$ to the harmonic restoring force, the drift term in the FFPE (16) changes to $-\partial(x-V) P(x, t) / \partial x$, and the exponential in expression (25) takes on the form $\exp \left(-\left[x-V-\left(x_{0}-V\right) e^{-t}\right] /\left[2\left(1-e^{-2 t}\right)\right]\right)$. As displayed in figure 9 , the strong persistence of the initial condition causes a highly asymmetric shape of the PDF, whereas the Brownian solution shown in dashed lines retains its symmetric Gaussian profile. Let us note again that a generalised Langevin picture would give rise to time-dependent coefficients, but would not change the Gaussian nature of the connected process in the harmonic potential.

Let us finally address the moments of the fractional Ornstein-Uhlenbeck process, equation (24). These can be readily obtained either from the Brownian result with the integral transformation (21), or from integration $\int d x x^{n}$. of the FFPE (16). For the first 


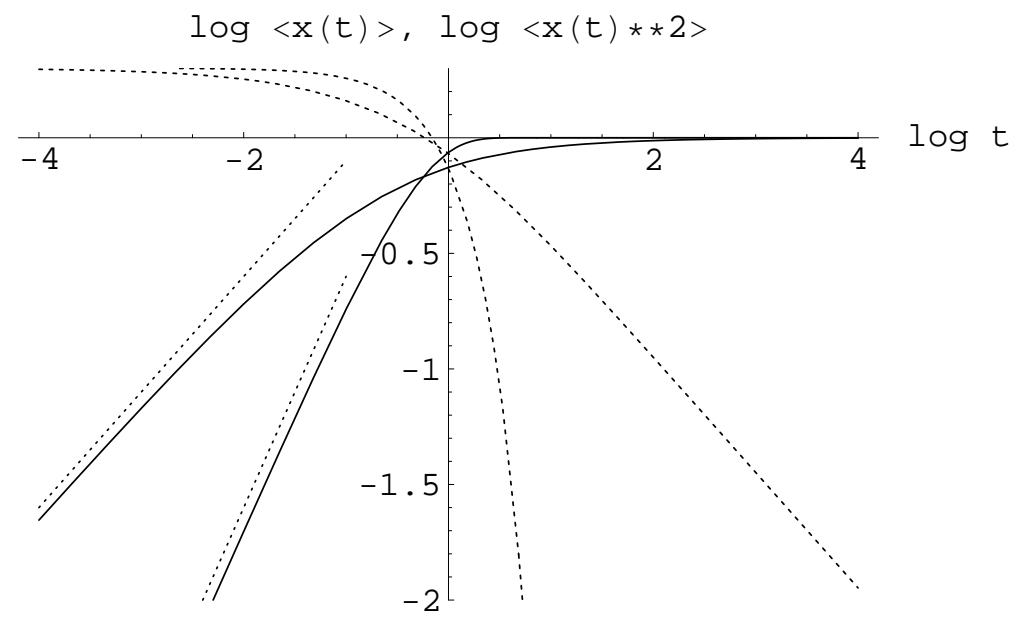

Figure 10. First ( $x_{0}=2$, dashed line) and second ( $x_{0}=0$, full line) moment of the fractional Ornstein-Uhlenbeck process $(\alpha=1 / 2)$, in comparison to the Brownian case. $\log _{10}-\log _{10}$ scale. The dotted straight lines show the initial (sub)diffusive behaviour with slopes $1 / 2$ and 1 , in the special case $x_{0}=0$ chosen for the second moment.

and second moments one obtains:

$$
\langle x(t)\rangle=x_{0} E_{\alpha}\left(-\frac{\omega^{2} t^{\alpha}}{\eta_{\alpha}}\right)
$$

and

$$
\left\langle x(t)^{2}\right\rangle=x_{\mathrm{th}}^{2}+\left(x_{0}^{2}-x_{\mathrm{th}}^{2}\right) E_{\alpha}\left(-\frac{2 \omega^{2} t^{\alpha}}{\eta_{\alpha}}\right),
$$

respectively. The first moment starts off at the initial position, $x_{0}$, and then falls off in a Mittag-Leffler pattern, reaching the terminal inverse power-law $\sim t^{-\alpha}$. The second moment turns from the initial value $x_{0}^{2}$ to the thermal value $x_{\mathrm{th}}^{2}=k_{B} T /\left(m \omega^{2}\right)$. In the special case $x_{0}=0$, the second moment measures initial force-free diffusion due to the initial exploration of the flat apex of the potential. We graph the two moments in figure 10 in comparison to their Brownian counterparts.

We note in passing that by optical tracking methods, it is in principle possible to obtain precise results for the Gaussian PDF of a single random walker in the equilibrium state, as demonstrated by [Oddershede et al (2002)]. It should therefore be possible to obtain more information also on anomalous processes, than through measurements of the mean squared displacement alone (particularly, due to the slow power-law relaxation of FFPE-governed processes it might be possible to monitor transient PDFs during the relaxation towards the Boltzmann equilibrium). We also note that for a particle connected to a membrane and experiencing in addition an optical tweezers potential the relaxation dynamics is closely related to the Mittag-Leffler decay [Granek and Klafter (2001)]. Finally, we mention that the fractional OrnsteinUhlenbeck process was investigated from the point of view of a time-dependent potential in reference [Tofighi (2003)]. 


\subsection{Fractional diffusion equations of distributed order}

There exist physical systems with so-called ultraslow diffusion of the logarithmic form

$$
\left\langle x^{2}(t)\right\rangle \sim \log ^{\kappa} t, \kappa>0,
$$

such as the famed Sinai diffusion $(\kappa=4)$ of a particle moving in a quenched random force field [Sinai (1982)], the motion of a polyampholyte hooked around an obstacle [Schiessel et al (1997)], and similarly in aperiodic environments [Igloi et al (1999)], in a family of iterated maps [Dräger and Klafter (2000)], as well as in a parabolic map [Prosen and Žnidaric (2001)].

Within the continuous time random walk theory, such 'strong anomalies' [Dräger and Klafter (2000)] can be described in terms of a waiting time PDF of the form [Havlin and Weiss (1990)]

$$
\psi(t) \sim \frac{\tau}{t \log ^{\kappa+1}(t / \tau)} .
$$

Obviously, the characteristic waiting time $\mathfrak{T}$ for this $\psi(t)$ diverges, although it is normalised. The corresponding propagator exhibits asymptotic exponential flanks of the form

$$
P(x, t) \sim \exp \left(-A \frac{|x|}{\log ^{\kappa / 2} t}\right) .
$$

For such strongly anomalous processes running off under the influence of an external potential, one would again like to have a description in terms of a dynamical equation. In fact, on the basis of distributed-order fractional operators [Caputo (1969), Caputo (2001), Chechkin et al (2003c)], the fractional equation

$$
\int_{0}^{1} \tau^{\beta-1} p(\beta)_{0} D_{t}^{\beta} P(x, t)=\left(\frac{\partial}{\partial x} \frac{V^{\prime}(x)}{m \eta}+K \frac{\partial^{2}}{\partial x^{2}}\right) P(x, t)
$$

was shown to lead to the desired logarithmic behaviour (29) in the force-free limit and with $p(\beta)=\kappa \beta^{\kappa-1}$ [Chechkin et al (2002b)]. Note that in the generalised Fokker-Planck operator we include properly generalised units of friction and diffusion coefficient.

The model equation (31), by construction, controls system relaxation towards the Boltzmann equilibrium, and fulfils the generalised Einstein-Stokes relation $K_{\mathrm{do}}=$ $k_{B} T /\left(m \eta_{\text {do }}\right)$. Moreover, one can show that it fulfils the linear response behaviour (17). We note that the mode relaxation, which is of Mittag-Leffler nature in the case of the regular (non-distributed) FFPE (16), for the distributed order case includes a logarithmic time dependence [Chechkin et al (2002b)].

\section{Superdiffusive processes}

Subdiffusive processes of the above kind can be physically understood in terms of the subordination to the corresponding Markov process, immanent in the multiple trapping

9 We should stress that the physics of equation (31) differs from Sinai diffusion, cf. [Chechkin et al (2002b)]. 
model with long-tailed waiting time PDF of the form (2). The solution corresponds to re-weighting of the Brownian solution with a sharply peaked kernel. In particular, the obtained PDFs relax towards the Boltzmann equilibrium, and they possess all moments if only the Brownian counterpart does (i.e., constant or confining potentials). Thus, the presence of the diverging characteristic waiting times does not change the quality (basin of attraction in a generalised central limit theorem sense) of the process in position $(x)$ space. In contrast, we will show in this section that for random processes with non-local jump lengths of the Lévy type, a priori surprising multimodal PDFs may arise and one observes a breakdown of the method of images. If the external potential is not steep enough, the moments diverge. Questions about the physical and thermodynamic interpretation of such processes arise. These points are going to be addressed in the following. We will first introduce the concept of Lévy flights (LFs) and discuss their formulation in terms of fractional equations. We then proceed to elaborate on some details concerning the abovementioned surprising features of LFs, before briefly addressing first results of a dynamic formulation of Lévy walks (LWs), the spatiotemporally coupled version of superdiffusive random processes, and their fractional formulation.

\subsection{Lévy flights}

LFs are Markov processes with broad jump length distributions with the asymptotic inverse power-law behaviour

$$
\lambda(x) \sim \frac{\sigma^{\mu}}{|x|^{1+\mu}}
$$

such that its variance diverges, $\mathfrak{X}^{2}=\int_{-\infty}^{\infty} \lambda(x) x^{2} d x=\infty$. This scale-free ${ }^{+}$form gives rise to the characteristic trajectories of LFs as shown in figure 11: In contrast to the 'area-filling' nature of a regular (Gaussian) random walk, an LF has a fractal dimension with exponent $\mu$ [Hughes (1995), Rocco and West (1999)], and consists of a self-similar clustering of local sojourns, interrupted by long jumps, at whose end a new cluster starts, and so on. This happens on all length scales, i.e., zooming into a cluster in turn reveals clusters interrupted by long sojourns. Thus, LFs intimately combine the local jump properties stemming from the centre part of the jump length distribution around zero jump length with strongly non-local, i.e., long-distance jumps, thereby creating slowly decaying spatial correlations, a signature of non-Gaussian processes with diverging variance [Hughes (1995), Bouchaud and Georges (1990), Lévy (1954), van Kampen (1981)]. Of course, also the Gaussian trajectory is self-similar, however, its finite variance prohibits the existence of long jumps separating local clusters.

Following along the lines pursued in the case of force-free subdiffusion, we describe LFs with a sharply peaked waiting time PDF $\psi(t)(\alpha=1)$ with finite characteristic waiting time $\mathfrak{T}$ and $\psi(u) \sim 1-u \tau$. The Fourier transform $\lambda(k)=\exp \left(-\sigma^{\mu}|k|^{\mu}\right) \sim$ $1-\sigma^{\mu}|k|^{\mu}$ of a Lévy stable jump length $\operatorname{PDF} \lambda(x)$ with asymptotic form (32) by

+ In the sense that there does not exist a variance of the jump length distribution. 


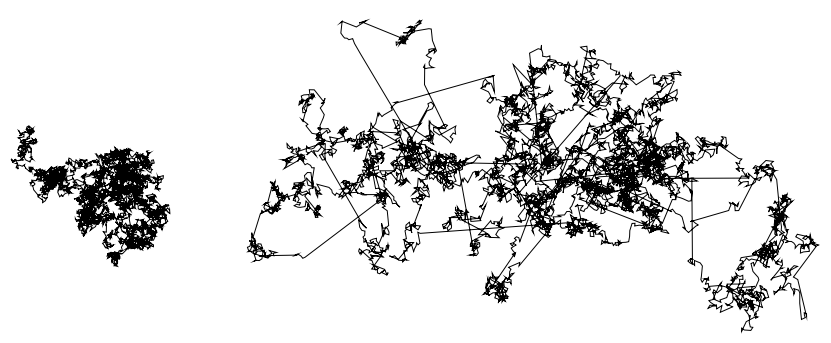

Figure 11. Lévy flight (right) of index $\mu=1.5$ and Gauss walk (left) trajectories with the same number $(\simeq 7000)$ of steps. The long sojourns and clustering appearance of the LF are distinct.

means of expression (A.1) produces a dynamical equation in Fourier-Laplace space, in which occurs the expression $|k|^{\mu} P(k, u)$ instead of the standard term $k^{2} P(k, u)$ in Gaussian diffusion. Let us for the moment define the fractional derivative in space through $F\left\{\frac{d^{\mu} g(x)}{d|x|^{\mu}}\right\} \equiv-|k|^{\mu} g(k)$ for $1 \leq \mu<2^{*}$, such that we infer the Lévy fractional diffusion equation [Compte (1996), Fogedby (1994), Honkonen (1996), Saichev and Zaslavsky (1997)]‡

$$
\frac{\partial}{\partial t} P(x, t)=K^{\mu} \frac{\partial^{\mu}}{\partial|x|^{\mu}} P(x, t),
$$

where we define in the analogous sense as above the generalised diffusion constant $K^{\mu} \equiv \sigma^{\mu} / \tau$ of (formal) dimension $\mathrm{cm}^{\mu} / \mathrm{sec} . \oint$ Again, equation (33) can be solved in closed form in terms of Fox $H$-functions [Metzler and Klafter (2000)]. Let us show that indeed equation (33) defines a Lévy stable law: Fourier transforming leads to the equation $\partial P(k, t) / \partial t=-|k|^{\mu} K^{\mu} P(k, t)$, which is readily integrated to yield $P(k, t)=\exp \left(-K^{\mu}|k|^{\mu} t\right)$, the characteristic function of a Lévy stable law [Gnedenko and Kolmogorov (1954), Lévy (1954)]. From the fractional operator (defined below) in equation (33), the symmetric, strongly non-local character of LFs becomes obvious. LFs were originally described by Mandelbrot, and formally the Fourier space analogue of equation (33) was discussed in reference [Seshadri and West (1982)] on the basis of a Langevin equation with Lévy noise, see below.

We note that due to the Markovian nature of LFs, a constant force/velocity $V$ can immediately be incorporated in terms of a moving wave variable, i.e., the solution of the LF in the presence of the drift $V$ defined by the equation

$$
\frac{\partial}{\partial t} P(x, t)=\left(\frac{\partial}{\partial x} V+K^{\mu} \frac{\partial^{\mu}}{\partial|x|^{\mu}}\right) P(x, t)
$$

is the solution $P_{V=0}(x, t)$ of equation (33) taken at position $x-V t$, i.e., $P_{V}(x, t)=$ $P_{V=0}(x-V t, t)$ [Metzler and Compte (2000), Metzler and Klafter (2000)].

* We do not pursue the case $0<\mu<1$ in what follows, although it follows the same reasoning.

$\ddagger$ Note that here we differ from our previous notation $-\infty D_{x}^{\mu}$ used in reference [Metzler and Klafter (2000)]. We follow here the one convention, which seems to become a standard for the space-fractional case, the Riesz-Weyl operators.

$\S$ NB: The waiting time PDF has the Laplace transform $\psi(u) \sim 1-u \tau$ for this Markovian case. 
4.1.1. Lévy fractional Fokker-Planck equation. LFs in the presence of an external potential $V(x)=-\int^{x} F\left(x^{\prime}\right) d x^{\prime}$ are described in terms of a different fractional FokkerPlanck equation, which we are going to call Lévy fractional Fokker-Planck equation (LFFPE) in the following. It has the simple form [Fogedby (1994), Fogedby (1994a), Fogedby (1998), Peseckis (1987)]

$$
\frac{\partial P}{\partial t}=\left(-\frac{\partial}{\partial x} \frac{F(x)}{m \eta}+K^{\mu} \frac{\partial^{\mu}}{\partial|x|^{\mu}}\right) P(x, t)
$$

where we encounter the fractional Riesz derivative defined through [Podlubny (1998), Samko et al (1993)]

$$
\begin{aligned}
& \frac{d^{\mu} f(x)}{d|x|^{\mu}}=\left\{\begin{array}{ll}
-\frac{\mathbf{D}_{+}^{\mu} f(x)+\mathbf{D}_{-}^{\mu} f(x)}{2 \cos (\pi \mu / 2)}, & \mu \neq 1 \\
-\frac{d}{d x} \mathbf{H} f(x), & \mu=1
\end{array},\right. \\
& \left(\mathbf{D}_{+}^{\mu} f\right)(x)=\frac{1}{\Gamma(2-\mu)} \frac{d^{2}}{d x^{2}} \int_{-\infty}^{x} \frac{f(\xi, t) d \xi}{(x-\xi)^{\mu-1}},
\end{aligned}
$$

and

$$
\left(\mathbf{D}_{-}^{\mu} f\right)(x)=\frac{1}{\Gamma(2-\mu)} \frac{d^{2}}{d x^{2}} \int_{x}^{\infty} \frac{f(\xi, t) d \xi}{(\xi-x)^{\mu-1}}
$$

for, respectively, the left and right Riemann-Liouville derivatives $(1 \leq \mu<2)$; for $\mu=1$, the fractional operator reduces to the Gilbert transform operator [Mainardi et al (2001)]

$$
(\mathbf{H} f)(x)=\frac{1}{\pi} \int_{-\infty}^{\infty} \frac{f(\xi) d \xi}{x-\xi}
$$

The Riesz operator has the convenient property

$$
f\left\{\frac{\partial^{\mu}}{\partial|x|^{\mu}} f(x) ; k\right\} \int_{-\infty}^{\infty} e^{i k x} \frac{\partial^{\mu}}{\partial|x|^{\mu}} f(x, t) d x \equiv-|k|^{\mu} f(k) .
$$

It should be noted that according to the LFFPE (35), it is only the diffusive term, which is affected by the Lévy noise. In contrast, the character of the drift remains unchanged, i.e., the external force is additive [Fogedby (1994), Fogedby (1994a), Fogedby (1998), Metzler et al (1999a), Metzler (2000)], as noted above for the case of a constant drift $V$.

Starting from the Feynman-Vernon path integral formulation of the influence functional [Feynman and Vernon (1963)], a characteristic functional, whose classical analogue corresponds to the Caldeira-Leggett equation for quantum Brownian motion, was established. By a Wigner transform for a Lévy source in the influence functional, the following Lévy fractional Klein-Kramers equation emerges [Lutz (2001)]:॥

$$
\frac{\partial}{\partial t} P(x, v, t)=-\frac{v}{m} \frac{\partial P}{\partial x}+V^{\prime}(x) \frac{\partial P}{\partial v}+\frac{\gamma}{m} \frac{\partial}{\partial v} v P+\gamma k_{B} T \frac{\partial^{\mu} P}{\partial|v|^{\mu}} .
$$

A similar equation was derived from a Langevin equation with Lévy noise in reference [Peseckis (1987)]. On the basis of a so-called quantum Lévy process, a Lévy fractional

\| In fact, the equation derived in [Lutz (2001)] also contains a term appearing in the case of asymmetric Lévy laws, which we do not consider herein. 
Klein-Kramers equation was obtained through random matrix methods in reference [Kusnezov et al (1999)], however, it carries a different friction term, as discussed in reference [Lutz (2003)]. Equation (41) was derived in reference [Metzler (2000)] from the generalised Chapman-Kolmogorov equation.

In equation (41), the fractional derivative is attached to the velocity $v$ of the particle. Thus, the corresponding LF-Rayleigh equation corresponds to a Lévy motion in an harmonic potential. As obvious from the result for the Lévy Ornstein-Uhlenbeck process reported in the next subsection, the solution $P(v, t)$ features a diverging mean squared displacement and in fact always remains a Lévy law with the same exponent $\mu$ [Jespersen et al (1999)]. In particular, the stationary state is a Lévy stable law of the same index $\mu$ [Seshadri and West (1982)]. We note, however, that this extreme Lévy behaviour is solely due to the linear friction inherent in equation (41). Due to the extremely large velocities attained by a Lévy flyer described by equation (41), i.e., to the divergence of the kinetic energy [Seshadri and West (1982)], the linear friction is in fact non-physical, and should be replaced by an expansion of the friction to higher order terms, $\gamma=\gamma(v)=\gamma_{0}+\gamma_{1} v^{2}+\ldots$ [Chechkin et al (2004)]. Such a velocitydependent friction was already discussed by Klimontovich and called dissipative nonlinearity [Klimontovich (1991)]. As we will see below, already the next higher term would produce a finite mean squared displacement of this more physical Lévy process. We also mention that another way to regularise Lévy flights is the spatiotemporal coupling of Lévy walks, which are briefly discussed below. We finally note that the velocity-average of equation (41) reduces directly to the LFFPE (35).

Although for the diverging moments, LFs may appear somewhat artificial, processes with diverging kinetic energy have been identified [Katori et al (1997)], and from a physics point of view are permissible in certain connections such as diffusion in energy space [Barkai and Silbey (1999), Zumofen and Klafter (1994)]. Moreover, LFs may be considered paradigmatic in the generalised central limit theorem sense and therefore deserve investigation. Not at least, they correspond to approximate schemes to more complex processes, like LWs.

4.1.2. Novel features of Lévy flights in superharmonic potentials. In reference [Jespersen et al (1999)], it was derived that the solution of the LFFPE in an harmonic potential field, $V(x)=\frac{1}{2} \omega x^{2}$, in Fourier space takes on the form

$$
P(k, t)=\exp \left(-\frac{\eta m K^{\mu}|k|^{\mu}}{\mu \omega}\left[1-e^{-\mu \omega t /(\eta m)}\right]\right),
$$

i.e., it is still a Lévy stable density, with the identical Lévy index $\mu$ as in the corresponding solution without external potential, and the stationary solution is $P_{\mathrm{st}}(x) \sim K^{\mu} \eta m /\left(\mu \omega|x|^{1+\mu}\right)$, in particular. Thus, an harmonic potential is not able to confine an LF such that its variance becomes finite, pertaining both to the time evolution and the stationary behaviour of the PDF. Equation (42) corresponds to the Lévy analogue of the Ornstein-Uhlenbeck process defined in equation (41) in phase space. 
From this perspective, it might seem a priori surprising that as soon as the external potential becomes slightly steeper than harmonic, the variance of the underlying LF becomes finite. However, this was demonstrated by [Chechkin et al (2002), Chechkin et al (2003a), Chechkin et al (2003b)] both analytically and numerically. We now briefly review the main features connected to such confined LFs. Before addressing these features for general $1<\mu<2$, we regard the case of a Cauchy flight $(\mu=1)$ in a quartic potential

$$
V(x)=\frac{b}{4} x^{4}
$$

whose stationary solution is exactly analytically solvable (for the more general cases we will draw on asymptotic arguments corroborated by numerical results). Thus, rewriting equation (35) with the quartic potential (43) in dimensionless coordinates [Chechkin et al (2002)],

$$
\frac{\partial P}{\partial t}=\left(\frac{\partial}{\partial x} x^{3}+\frac{\partial^{\mu}}{\partial|x|^{\mu}}\right) P(x, t)
$$

one can immediately derive the stationary $\left(d P_{\mathrm{st}}(x) / d t=0\right)$ solution

$$
P_{\text {st }}(x)=\pi^{-1} \frac{1}{1-x^{2}+x^{4}}
$$

which is remarkable in two respects: (i) the asymptotic power-law $P_{\mathrm{st}}(x) \sim x^{-4}$ falls off steeper than the Lévy stable density with index $\mu$, and the variance in fact converges, i.e., the process leaves the basin of attraction of the generalised central limit theorem; and (ii) the PDF (45) is bimodal, i.e., it exhibits two maxima at $x_{\max }= \pm 1 / \sqrt{2}$ [Chechkin et al (2002)], see figure 12. This bimodality becomes increasingly pronounced when the Lévy index approaches the Cauchy case, $\mu=1$ [Chechkin et al (2002)]. We note that the numerical solution procedures we employ for determining the PDF defined by the LFFPE is mainly based on the Grünwald-Letnikov representation of the fractional Riesz derivative [Podlubny (1998), Gorenflo (1997), Gorenflo et al (2002)], details of which are also described in [Chechkin et al (2003a)]; see also reference [Lynch et al (2003)].+

Let us pursue somewhat further the incapability of an harmonic potential to confine an LF in contrast to a quartic potential and give rise to multimodal states, some of which are transient. To this end, consider the combined potential [Chechkin et al (2003b)]

$$
V(x)=\frac{a}{2} x^{2}+\frac{b}{4} x^{4}
$$

By rescaling of the LFFPE (35) with the potential (46) according to $x \rightarrow x / x_{0}, t \rightarrow t / t_{0}$, with $x_{0} \equiv(m \eta D / b)^{1 /(2+\mu)}$ and $t_{0} \equiv x_{0}^{\mu} / D$, and $a \rightarrow a t_{0} / m \eta$, we obtain the normalised form

$$
\frac{\partial P}{\partial t}=\left(\frac{\partial}{\partial x}\left[x^{3}+a x\right]+\frac{\partial^{\mu}}{\partial|x|^{\mu}}\right) P(x, t)
$$

+ We note that bifurcation to a bimodal state may be obtained from a linear Langevin equation with mulitplicative noise term and time-dependent drift [Fa (2003)], another, yet different scenario towards multimodal states. Compare also section 4.2. 

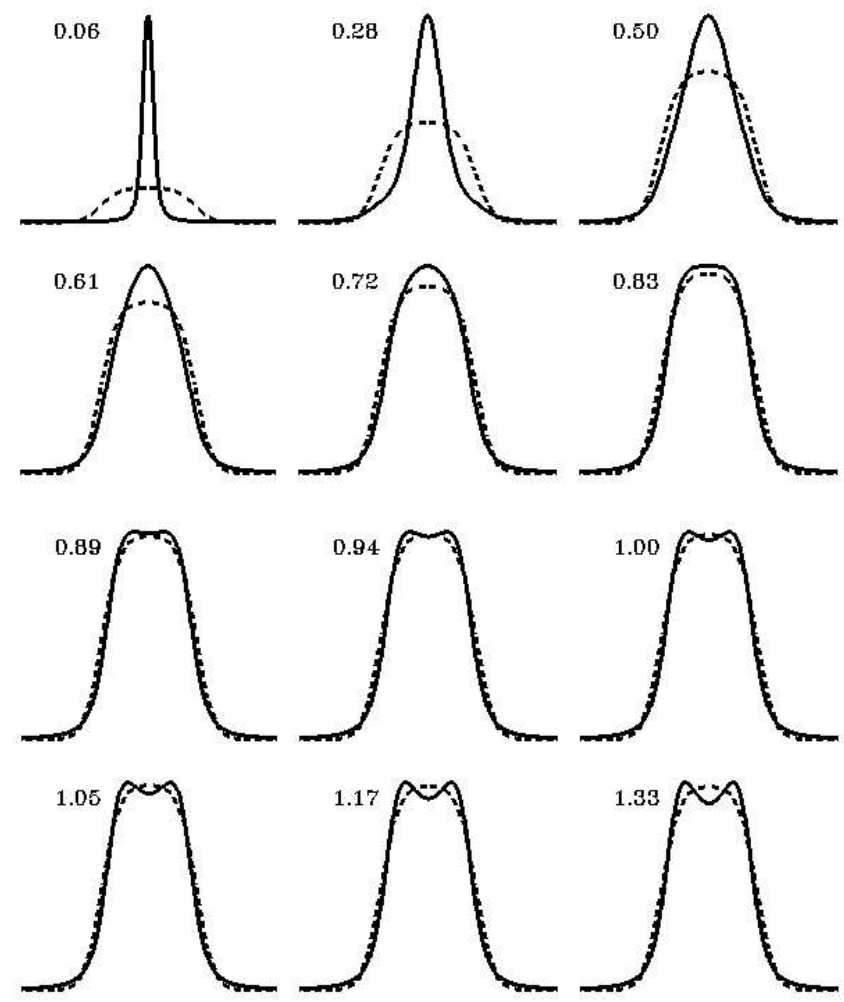

Figure 12. Time evolution of the PDF governed by the LFFPE (35) in a quartic potential, starting from $P(x, 0)=\delta(x)$, with Lévy index $\mu=1.2$. The dashed line indicates the Boltzmann distribution from the Gaussian process in an harmonic potential.

for the LFFPE (35). A detailed analysis, both analytically and numerically, reveals that there exists a critical magnitude of the relative harmonicity strength $a, a_{c} \simeq 0.794$, below which a bimodal state exists [Chechkin et al (2003b), Chechkin et al (2003a)]. Dynamically, starting from a monomodal initial condition such as a $\delta$-peak in the centre of the potential, it turns out that there exists a critical time $t_{c}$ at which the PDF develops bimodality (figure 12). This turnover can be studied similarly to the critical harmonicity strength, and the result is shown in figure 13 at the top: For times $t>t_{c}$, a bimodal state spontaneously comes into existence, corresponding to a dynamical bifurcation. However, for all potentials $V(x)$ containing a term $\propto|x|^{2+c}$ with $c>0$, the variance becomes finite for all $t>0$ [Chechkin et al (2002)].

Given the potential

$$
V(x)=\frac{a}{c}|x|^{c} \quad \therefore \quad c \geq 2
$$

there exists an additional, transient trimodal state in the case $c>4$, an example of which is depicted in figure 14. In this case, that is, the relaxation of the peak of the initial condition overlaps with the building up of the two side-maxima, which will eventually give rise to the terminal bimodal PDF. In figure 13 at the bottom, the size of the maxima and their temporal evolution are shown. 

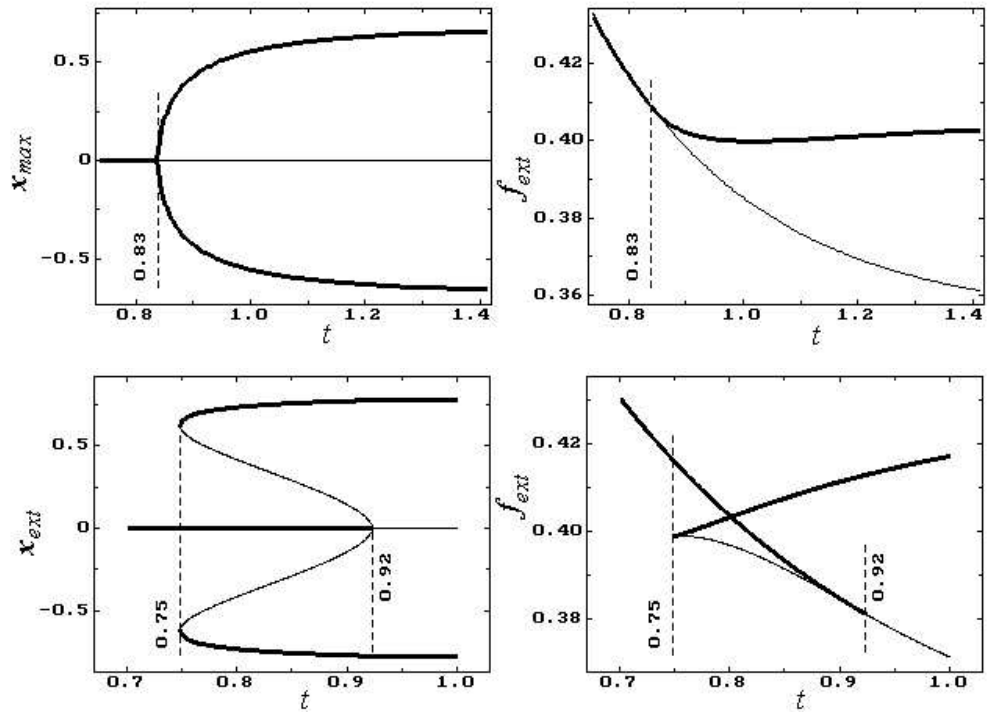

Figure 13. Bifurcation diagrams. Upper panel: For the case $c=4.0, \mu=1.2$, on the left part the thick lines show the location of the maximum, which at the bifurcation time $t_{12}=0.84 \pm 0.01$ turns into two maxima; right part: the value of the PDF in the maxima location (thick line) and the value in the minimum at $x=0$ (thin line). Lower panel: For the case $c=5.5$ and $\mu=1.2$, on the left part positions $x_{\max }$ of the maxima (global and local, thick lines); the thin lines indicate the positions of the minima (at the first bifurcation time, there is a horizontal tangent at the site of the two emerging off-centre maxima. The bifurcation times are $t_{13}=0.75 \pm 0.01$ and $t_{32}=0.92 \pm 0.01$. Right part: values of the PDF at the maxima (thick lines); the thin line indicates the value of the $\mathrm{PDF}$ in the minima.
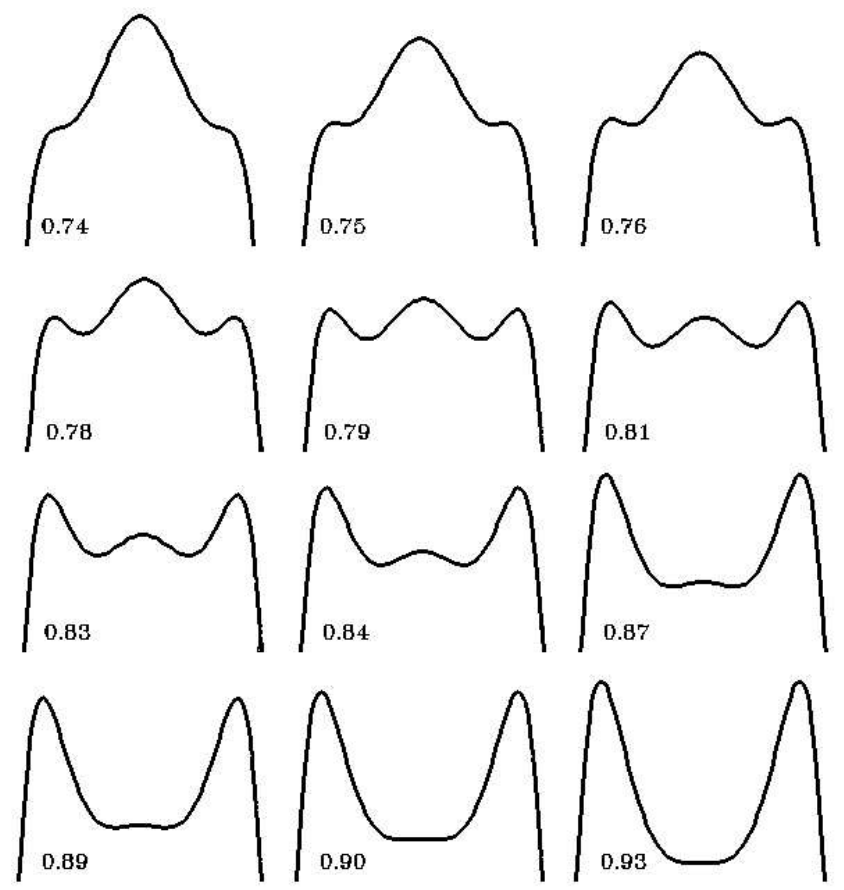

Figure 14. Same as in 12, in a superharmonic potential (48) with exponent $c=5.5$, exhibiting a trimodal structure. 
Similar a priori surprising effects of LFs were found in periodic potentials, in which LFs turn out to be delicately sensitive. For instance, there has been revealed a rich band structure in the Bloch waves described by an LFFPE and its associated fractional Schrödinger-type equation [Brockmann and Geisel (2003)].

4.1.3. Lévy flights and thermal (Boltzmann) equilibrium. Above definition (35) of the LFFPE describes a process far from thermal (Boltzmann) equilibrium. In particular, it does not fulfil the linear response theorem in the form (17) known from Gaussian and subdiffusive processes, due to the divergence of the second moment. However, it still underlies the physical concept of additivity of drift and diffusive terms manifested in the fact that for a constant force field $F(x)=m \eta V$, the solution of the LFFPE (35) is given by the propagator at zero force, $P_{F=0}(x-V t, t)$, taken at the wave variable $x-V t$ [Jespersen et al (1999), Metzler et al (1998), Metzler and Klafter (2000)]. Above, it was shown that this additive combination of drift and diffusivity produces solutions with converging variance and multimodal properties in superharmonic potentials.

It is interesting to see that one can consistently obtain an equation that describes LFs in the absence of a force field, but which relaxes towards classical thermal equilibrium for any non-trivial external field. This equation is based on a different weighting, introduced through a subordination, leading to the 'exponent-fractional' equation [Sokolov et al (2001)]

$$
\frac{\partial}{\partial t} P(x, t)=-K^{\mu}\left(-\frac{\partial^{2}}{\partial x^{2}}+\frac{\partial}{\partial x} \frac{F(x)}{k_{B} T}\right)^{\mu} P(x, t),
$$

where the $\mu$ th power of the Fokker-Planck operator is interpreted in Fourier-Laplace space, i.e., equation (49) acquires the form $\partial P / \partial t=-K^{\mu}\left(\mathrm{i} k f /\left[k_{B} T\right]+k^{2}\right)^{\mu} P(k, t)$ [Sokolov et al (2001)]. As shown in [Sokolov et al (2001)], the PDF $P(x, t)$ relaxes exponentially towards the regular Boltzmann equilibrium PDF, and is therefore qualitatively different from the LFFPE (35) discussed above. The eigenvalues of $\lambda_{n}^{\text {ef }}$ of equation (49) are related to those of the regular Fokker-Planck equation $\left(\lambda_{n}\right)$ by $\lambda_{n}^{\text {ef }}=-\left(-\lambda_{n}\right)^{\mu}$, the eigenfunctions coinciding, and the relaxation of moments is exponential, $\propto \exp \left(-\right.$ const. $\left.\left|\lambda_{n}^{\text {ef }}\right|^{\mu} t\right)$ [Sokolov et al (2001)]. In particular, the process leading to the modified LF-equation (49) does not possess a direct interpretation of continuous time random walks (but it can be related to the Chapman Kolmogorov equation). An interesting question will be to determine the corresponding Langevin picture of such process.

\subsection{Bi-fractional transport equations}

The coexistence of long-tailed forms for both jump length and waiting time PDFs was investigated within the CTRW approach in reference [Zumofen and Klafter (1995a)], discussing in detail the laminar-localised phases in chaotic dynamics. In a similar way, the combination of the long-tailed waiting time PDF (2) with its jump length analogue (32) leads to a dynamical equation with fractional derivatives in respect to both time and 


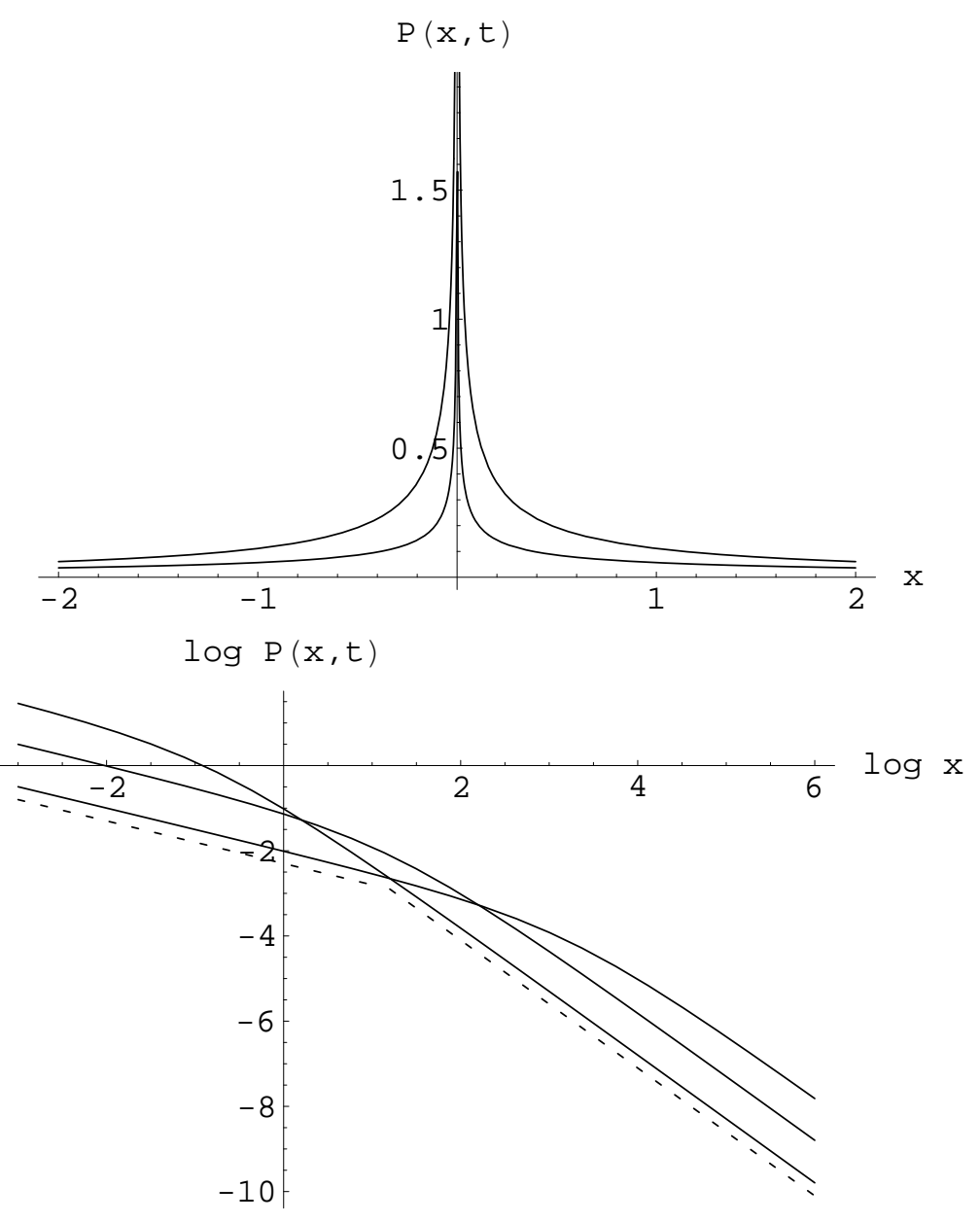

Figure 15. Subdiffusion for the neutral-fractional case $\alpha=\mu=1 / 2$. Top: Linear axes, dimensionless times $t=2$, 20. Bottom: Double-logarithmic scale, dimensionless times, $t=0.1,10,1000$. The dashed lines in the bottom plot indicate the slopes $-1 / 2$ and $-3 / 2$. Note the divergence at the origin.

space [Luchko et al (1998), Mainardi et al (2001), Metzler and Nonnenmacher (2002), West and Nonnenmacher (2001)]:

$$
\frac{\partial}{\partial t} P(x, t)=K_{\alpha}^{\mu} D_{t}^{1-\alpha} \frac{\partial^{\mu}}{\partial|x|^{\mu}} P(x, t) .
$$

This equation can in fact be extended to cover the superdiffusive, sub-ballistic domain up to the wave equation (for $\mu=2$ ) [Metzler and Klafter (2000c)], and under the condition $1 \leq \alpha \leq \mu \leq 2$ in general [Mainardi et al (2001)]. A closed form solution can be found in terms of Fox $H$-functions [Metzler and Nonnenmacher (2002)].

A special case of equation (50) is the 'neutral-fractional' case $\alpha=\mu$ [Mainardi et al (2001)]. In this limit, one can obtain simple reductions of the $H$-function solution, in the following three cases [Metzler and Nonnenmacher (2002)]:

(i) Cauchy propagator $\alpha=\mu=1$ :

$$
P(x, t)=\frac{1}{2 \pi K_{1}^{1} t} \frac{1}{1+x^{2} /\left(K_{1}^{1} t\right)}
$$




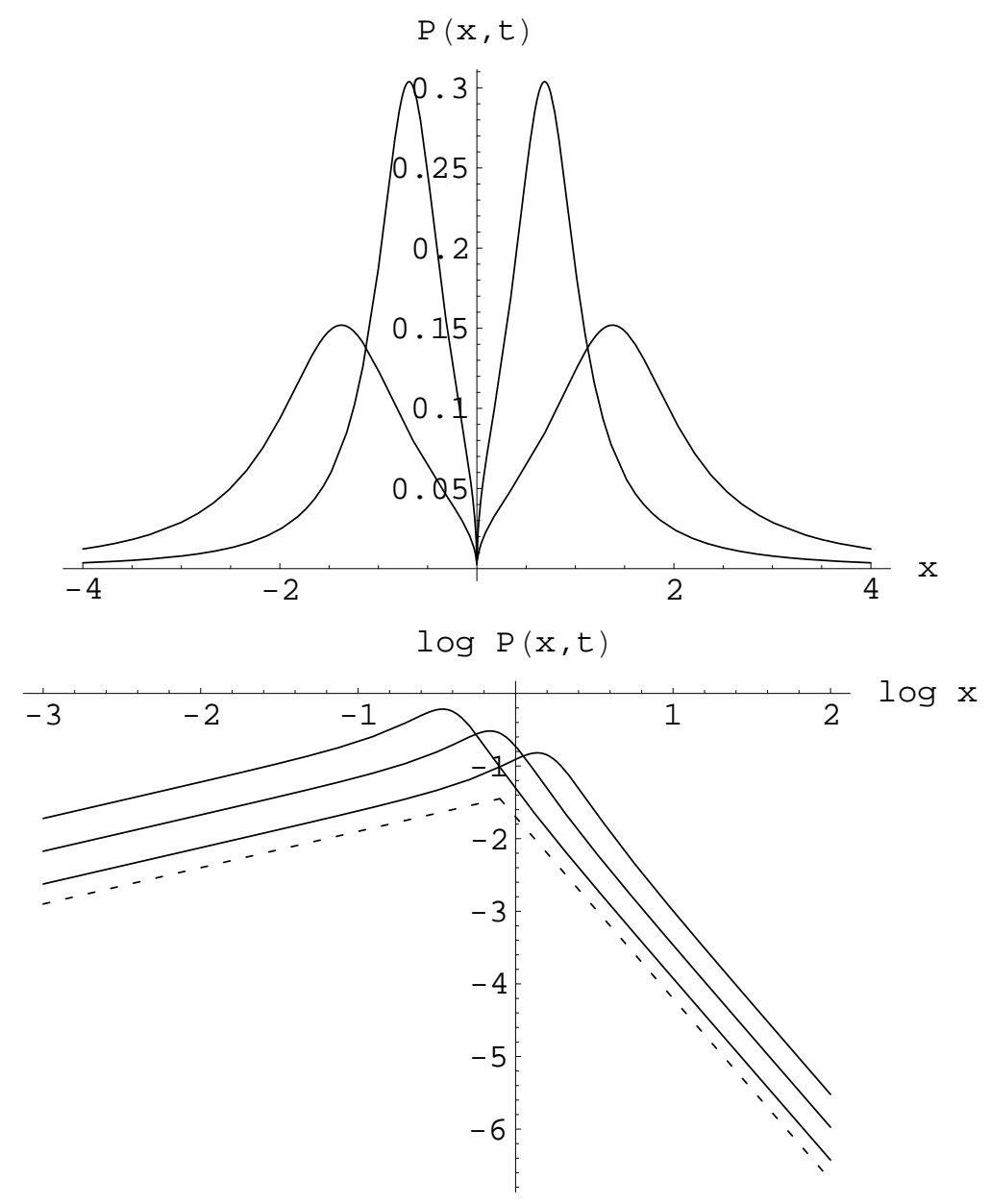

Figure 16. Superdiffusion for the neutral-fractional case $\alpha=\mu=3 / 2$. Top: Linear axes, dimensionless times $t=1$ and 2. Bottom: double-logarithmic scale drawn for the dimensionless times $0.5,1$ and 2 . The dashed lines in the bottom plot indicate the slopes $1 / 2$ and $-5 / 2$. Note the complete depletion at the origin.

with the long-tailed asymptotics $P(x, t) \sim(2 \pi)^{-1} x^{-2}$. The Cauchy propagator converges to $\left(2 \pi K_{1}^{1} t\right)^{-1}$ at $x=0$.

(ii) The case $\alpha=\mu=1 / 2$ (figure 15 ):

$$
P(x, t)=\frac{2}{|x|} \frac{z^{1 / 2}}{\sqrt{2} \pi+2 \pi z^{1 / 2}+\sqrt{2} \pi z} \therefore z=\frac{|x|}{2\left(K_{1 / 2}^{1 / 2}\right)^{2} t} .
$$

At the origin, this PDF diverges $\sim|x|^{-1 / 2}$, while for large $|x|$, it decays like $\sim|x|^{-3 / 2}$.

(iii) The case $\alpha=\mu=3 / 2$ (figure 16):

$$
P(x, t)=\frac{\sqrt{2}}{3 \pi|x|} \frac{z^{3 / 2}+z^{3}+z^{9 / 2}}{1+z^{6}} \therefore z=\frac{2^{1 / 3}|x|}{\left(K_{3 / 2}^{3 / 2}\right)^{2 / 3} t} .
$$

In this case, the PDF shows complete depletion at the origin, exhibiting a $\sim|x|^{1 / 2}$ square root behaviour close to $|x|=0$, and it has the inverse power-law decay $\sim|x|^{-5 / 2}$ 
for $|x| \rightarrow \infty$. This solution also exhibits a bimodal PDF, known from equation (9) in the superdiffusive sub-ballistic regime $(1<\alpha<2)$ [Metzler and Klafter (2000c), West et al (1997)]. This superdiffusive feature comes from the interpretation of the process as a memory version of the wave equation, i.e., to a propagative contribution [Metzler and Klafter (2000c)]. It is therefore completely different from the bimodal structure in confined LFs discussed above.

We note that bi-fractional diffusion equations were also discussed in references [Barkai (2002), Saichev and Zaslavsky (1997), Baeumer et al (2003), Hughes (2002), Gorenflo et al (2002a), Uchaikin (2002)]. A bi-fractional Fokker-Planck equation with a power-law dependence $\propto|x|^{-\theta}(\theta \in \mathbb{R})$ of the diffusion coefficient was studied in references [Fa and Lenzi (2003), Lenzi et al (2003)].

\subsection{Lévy walks}

Lévy walks (LWs) correspond to the spatiotemporally coupled version of continuous time random walks. The waiting time and jump length PDFs, that is, are no longer decoupled but appear as conditional in the form $\psi(x, t) \equiv$ $\lambda(x) p(t \mid x)$ (or $\psi(t) \tilde{p}(x \mid t)$ ) [Klafter et al (1987)]. In particular, through the coupling $p(t \mid x)=\frac{1}{2} \delta\left(|x|-v t^{\nu}\right)$, one introduces a generalised velocity $v$, which penalises long jumps such that the overall process, the LW, attains a finite variance and a PDF with two spiky fronts successively exploring space [Zumofen and Klafter (1993), Klafter and Zumofen (1994)]. Thus, LWs have similar properties to generalised Cattaneo/telegraphers' equation-type models [Compte and Metzler (1997), Metzler and Compte (1999), Metzler and Nonnenmacher (1998)].

On the basis of the latter fractional equations, formulations were obtained for the description of LWs in the presence of non-trivial external force fields, with the same restriction to lower order moments in respect to an LW process [Barkai and Silbey (2000), Metzler and Sokolov (2002)]. Recently, however, a coupled fractional equations was reported [Sokolov and Metzler (2003)], which describes a forcefree LW exactly. Thus, it was shown that the fractional version of the material derivative $\partial / \partial t \pm \partial / \partial x$

$$
d_{ \pm}^{\beta} P(x, t) \equiv{ }_{0} D_{t}^{\beta} P(x \pm t, t)
$$

defined in Fourier-Laplace space through

$$
f\left\{\mathscr{L}\left\{d_{ \pm}^{\beta} f(x, t) ; u\right\} ; k\right\} \equiv(u \pm i k)^{\beta} f(k, u),
$$

( $f$ acts on $x$ and $\mathscr{L}$ on $t$ ) replaces the uncoupled fractional time operators introduced in the previous work, compare also the detailed discussion of LW processes in reference [Zumofen and Klafter (1993)]. Although one may argue for certain forms [Sokolov and Metzler (2003)], there is so far no derivation for the incorporation of general external force fields in the coupled formalism. A question of particular interest is whether LWs in non-trivial external fields relax towards a stationary solution or not, 
compare [Metzler and Sokolov (2002)]. We note that a very similar fractional approach to LWs was suggested by [Meerschaert et al (2002)] virtually simultaneously.

\section{Applying fractional dynamics}

The interesting quantity of many dynamical processes is the PFD and its associated mean time, to arrive at or cross a certain point after having started someplace else in the system. This is the problem of first passage, which for Brownian motion is identical to the problem of first arrival. In the presence of subdiffusion, the first passage time problem corresponds to a subordination of the analogous Brownian problem, and can be solved with the same tools. The major difference is the divergence of the mean first passage time (MFPT) T. In contrast, for LFs, we will report that the method of images breaks down, and the details of the process (especially the Lévy index) enter only marginally into the first passage time density (FPTD). Apart from the first passage, the nature of the underlying diffusion process naturally defines the detailed properties of the associate diffusion-reaction problem. We are going to discuss these two fundamental applications of (anomalous) diffusion processes in the following.

\subsection{First passage time processes and boundary conditions}

The firing of neurons [Ben-Yacov (2002), Gerstner and Kistler (2002)], diffusionlimited aggregation [Vicsek (1991)], hydrological breakthrough [Kirchner et al (2000), Scher et al (2002)], the passage of a biomolecule through a membrane nanopore [Bates et al (2003), Metzler and Klafter (2003)], the fluctuation behaviour of single strand bubbles in DNA double helices [Hanke and Metzler (2003)], the encounter of two independently diffusion particles [von Smoluchowski (1916), von Smoluchowski (1916a)], the rebinding of a ligand to a protein [Iben et al (1989)], the switching of topological molecules [Metzler (2001a)], or the electrical current caused by anomalously moving charge carriers in amorphous semiconductors [Pfister and Scher (1977), Pfister and Scher (1978)], etc., can be mapped onto the problem of calculating the FPTD, and the associated MFPT [Redner (2001)], an approach originally dating back to [Schrödinger (1915)].

In the regular, Brownian domain of diffusion, the unbiased passage for a process with initial condition $P_{0}(x)=P(x, 0)=\delta\left(x-x_{0}\right)$ is described by the FPTD

$$
p(t)=\frac{x_{0}}{\sqrt{4 \pi K t^{3}}} \exp \left(-\frac{x_{0}^{2}}{4 K t}\right),
$$

which defines the probability $p(t) d t$ for the particle to arrive at $x=0$ during the time interval $t, \ldots, t+d t$. Its long-time behaviour corresponds to the $3 / 2$ power-law behaviour

$$
p(t) \sim \frac{x_{0}}{\sqrt{K}} t^{-3 / 2}
$$

In particular, we note that even for Brownian processes there are natural cases when the characteristic time diverges. Here, that is, the MFPT $T=\int_{0}^{\infty} p(t) t d t=\infty$. 
In a finite box of size $L$, the Brownian first passage time problem has an exponential tail, with increasingly quicker decay on increasing mode number,

$$
p_{L}(t)=\frac{\pi K}{L^{2}} \sum_{n=0}^{\infty}(-1)^{n}(2 n+1) \exp \left(-K \frac{(2 n+1)^{2} \pi^{2}}{4 L^{2}} t\right) .
$$

This solution for the FPTD corresponds to an initial condition in the centre of a box with two absorbing boundaries. The MFPT becomes $T=L^{2} /(2 K)$.

The third case of interest here is the first passage in a semi-infinite domain in the presence of a constant bias $V$. In the corresponding FPTD

$$
p_{V}(t)=\frac{x_{0}}{\sqrt{4 \pi K t}} \exp \left(-\frac{\left(x_{0}-V t\right)^{2}}{4 K t}\right)
$$

the drift term $V^{2} t^{2}$ in the exponential outweighs the diffusive $1 /(K t)$ part, and causes an exponential decay. One finds the MFPT $T=x_{0} / V$, i.e., the average first passage time matches exactly the classical motion with constant velocity $V$.

Let us now investigate what changes are coming about in the presence of transport anomalies of subdiffusive and LF nature.

5.1.1. Subdiffusion. The first passage of a diffusing particle through a point $x_{f}$ in processes characterised by a jump length PDF with finite variance $\mathfrak{X}^{2}$ can always be mapped on the problem of putting an absorbing boundary at that point $x_{f}$ and calculating the negative time derivative of the survival probability. A natural way of constructing the FPTD in such a case is the method of images [Redner (2001)] going back to Lord Kelvin. For instance, on the semi-infinite domain, this method prescribes to mirror the unrestricted propagator with initial condition $x_{0}$ at the point $x_{f}$, and turning this image negative. The image solution is then the sum of both PDFs. Thus, the part of the PDF which has imaginarily crossed the absorbing boundary at $x_{f}$ at some time $t$, is subtracted from the original PDF. In our example of the semi-infinite domain with $x_{f}=0$, the image solution becomes $Q(x, t)=P\left(x, t ; x_{0}\right)-P\left(x, t ;-x_{0}\right)$. Thus, one readily obtains $Q(0, t)=0$, as it should. The integral $S(t)=\int_{0}^{\infty} Q(x, t) d x$ is the survival probability. Its negative derivative $p(t)=-d S(t) / d t$ is then the FPTD. In a similar manner, the images method can be employed to solve the (sub)diffusion in a box, or in the presence of a constant bias [Metzler and Klafter (2000d), Redner (2001)].

Another method is based on the generalised Laplace transformation (21), which is equally valid for the position-average of $P(x, t)$, in the present language the survival probability. Thus, we can relate the Markovian $\left(S_{M}\right)$ and subdiffusive $\left(S_{\alpha}\right)$ results through

$$
S_{\alpha}(u)=u^{\alpha-1} S_{M}\left(u^{\alpha}\right)
$$

corresponding to the relation through equations (20) and (21). As the FPTD is the (negative) time derivative of the survival probability $S$, the Brownian and subdiffusive FPTDs are not connected by relation (21). However, it is straightforward to show that the latter fulfil the scaling relation

$$
p_{\alpha}(u)=p_{M}\left(u^{\alpha}\right)
$$


in Laplace space. In the time domain, this corresponds to another generalised Laplace transformation of the kind (21), but with the one-sided Lévy density $\mathscr{L}^{-1}\left\{\exp \left(-s u^{\alpha}\right) ; t\right\} \sim t^{-1-\alpha}$ as kernel.

The essential property of subdiffusive first passage time problems lies in the fact that the long-tailed nature of the waiting time PDF translates into the FPTD itself. The MFPT, that is, diverges both in the absence of a bias and under a constant drift, pertaining to both finite as well as semi-infinite domains [Metzler and Klafter (2000d), Rangarajan and Ding (2000), Rangarajan and Ding (2000a), Rangarajan and Ding (2003), Scher et al (2002), Barkai (2001)]. † For the three cases of first passage time problems, we obtain the following subdiffusive generalisations:

(i) For subdiffusion in the semi-infinite domain with an absorbing wall at the origin and initial condition $P(x, 0)=\delta\left(x-x_{0}\right)$ it was found that [Metzler and Klafter (2000d)]

$$
p(t) \sim \frac{x_{0}}{|\Gamma(-\alpha / 2)| K_{\alpha}^{1 / 2}} t^{-1-\alpha / 2}
$$

i.e., the decay becomes a flatter power-law than in the Markovian case (57).

(ii) Subdiffusion in the semi-infinite domain in the presence of an external bias $V$ falls off faster, but still in power-law manner [Barkai (2001), Metzler and Klafter (2003), Scher et al (2002)]:

$$
p(t) \sim t^{-1-\alpha} .
$$

In strong contrast to the biased Brownian case, we now end up with a process whose characteristic time scale diverges. This is exactly the mirror of the multiple trapping model, i.e., the classical motion events become repeatedly interrupted such that the immobilisation time dominates the process.

(iii) Subdiffusion in a finite box [Metzler and Klafter (2000d)]: $\ddagger$

$$
p(t) \sim t^{-1-\alpha},
$$

i.e., this process leads to the same scaling behaviour for longer times as found for the biased semi-infinite case (ii). Effectively, that is, the drift towards the absorbing boundary outweighs the diffusion, as remarked in the Brownian case above, and acts as a reflecting boundary.

The latter two results should be compared to the classical Scher-Montroll finding for the FPTD of biased motion in a finite system of size $L$ with absorbing boundary condition. In that case, the FPTD exhibits two power-laws (compare figure 17)

$$
p(t) \sim \begin{cases}t^{\alpha-1}, & t<\tau \\ t^{-1-\alpha}, & t>\tau\end{cases}
$$

$\dagger$ Note that for both subdiffusive ( $\mathfrak{T}$ diverges) and LFs ( $\mathfrak{X}^{2}$ diverges) systems it may be dangerous to invoke results from the $x, t$-scaling found in the mean squared displacement $\left\langle\Delta x^{2}(t)\right\rangle \sim t^{\alpha}$, from which erroneously a finite MFPT could be predicted, or inferred at all, respectively. Compare [Gitterman (2000), Yuste and Lindenberg (2004)] and [Li and Wang (2003), Metzler and Sokolov (2004)], as well as reference [Denisov et al (2003), Reigada et al (2002)].

$\ddagger$ It does not matter whether both boundaries are absorbing, or one is reflective. 


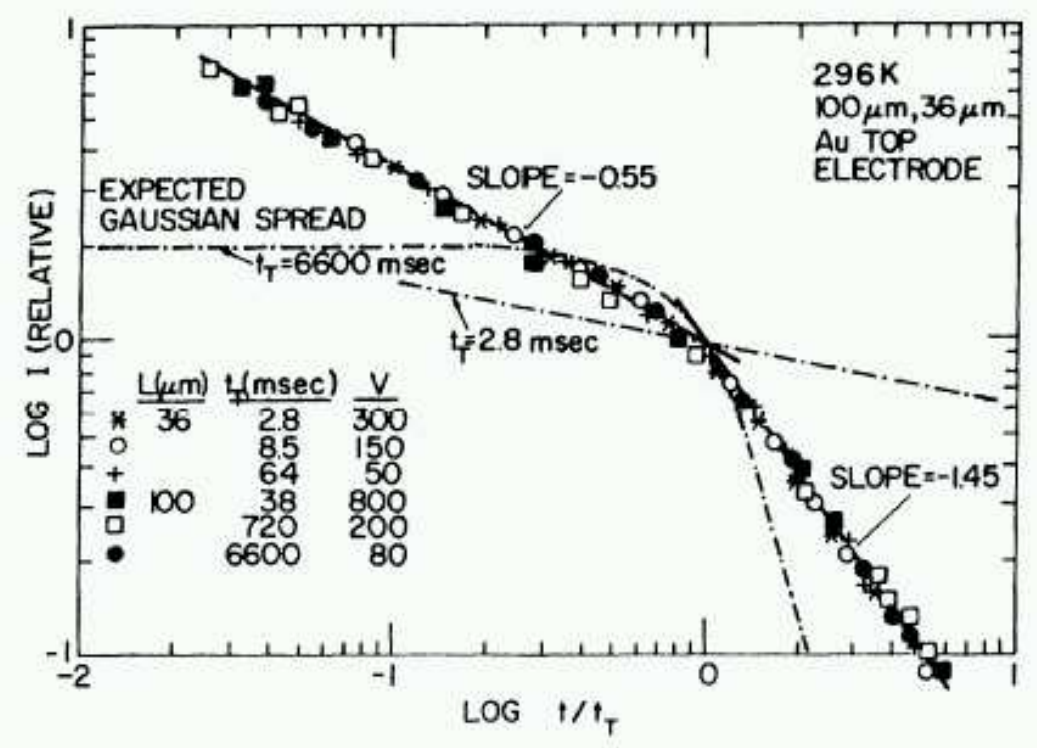

Figure 17. Universality of the electrical current shape as function of time, for various driving voltages as sample sizes. The slopes of the two power-laws add up to 2 . Note that beautiful data collapsing after rescaling [Pfister and Scher (1977)]. .

the sum of whose exponents equals -2 [Pfister and Scher (1977), Pfister and Scher (1978), Scher et al (2002a), Scher et al (2002)]. Here, $\tau$ is a system parameter dependent time scale [Pfister and Scher (1978)]. In figure 17 we also find the anomalous diffusion modelling beautifully proved by the experimental data [Pfister and Scher (1977), Pfister and Scher (1978), Scher and Montroll (1975)]. As shown in figure 17, a Brownian drift-diffusion model could not reasonably reproduce the data (which in that case would also be non-universal in the sense that it would not exhibit the observed data collapsing). Note that the anomalous transport behaviour also correctly describes other system features, such as the size-dependent mobility. These investigations were the first, and highly successful, application of continuous time random walk dynamics.

5.1.2. Lévy flights. The first passage time problem for LFs might naively be considered simpler than the corresponding subdiffusive problem, keeping in mind that LFs are Markovian. Moreover, their dynamical equation is linear. It might therefore be tempting to apply the method of images to infer the FPTD for an LF. Such an approach was in fact pursued in reference [Montroll and West (1976)] for a finite domain, and with similar methods in reference [Gitterman (2000)]. In reference [Buldyrev et al (2001)], eigenfunctions of the full-space LFFPE were obtained, from which the FPTD was determined. These methods lead to results for the FPTD in the semi-infinite domain, whose long-time behaviour is explicitly dependent on the Lévy index, such that the FPTD would actually decay slower than under Brownian conditions. Given the much quicker exploration of space of LFs, this is a counterintuitive result. In fact, it was shown by Sparre Andersen that any symmetric jump length PDF gives rise to a 
decay $\sim n^{-3 / 2}$ of the FPTD with the number $n$ of steps [Sparre Andersen (1953), Sparre Andersen (1954)]. For any Markov process, the analogous continuous time behaviour can be obtained through $n \propto t$, and is according to the Sparre-Andersen theorem given by equation (57). Thus, an LF necessarily has to fulfil the SparreAndersen universality, fully independent of the Lévy index. $\neq$ We note that an analogous result was proved in reference [Frisch and Frisch (1995)] for the special case in which an absorbing boundary is placed at the location of the starting point of the LF at $t>0$, and numerically corroborated in reference [Zumofen and Klafter (1995)].

The inadequacy of the images method can indeed be understood from the Lévy fractional diffusion equation (33). There, reflecting the strongly non-local nature of LFs the fractional Riesz operator stretches its integration from $-\infty$ to $+\infty$. However, given an absorbing boundary condition at $x=0$ with an initial condition at $x_{0}>$ 0 , the solution necessarily has to vanish on the negative semi-axis. The correct dynamical equation in the presence of the boundary condition should therefore read [Chechkin et al (2003)]

$$
\frac{\partial f(x, t)}{\partial t}=\frac{D}{\kappa} \frac{\partial^{2}}{\partial x^{2}} \int_{0}^{\infty} \frac{f\left(x^{\prime}, t\right)}{\left|x-x^{\prime}\right|^{\mu-1}} \mathrm{~d} x^{\prime} \equiv \frac{\partial^{2}}{\partial x^{2}} \mathcal{F}(x, t)
$$

in which the fractional integral is truncated to the semi-infinite interval. After Laplace transformation and integrating over $x$ twice, one obtains

$$
\int_{0}^{\infty} K\left(x-x^{\prime}, s\right) f\left(x^{\prime}, s\right) d x^{\prime}=\left(x-x_{0}\right) \Theta\left(x-x_{0}\right)-x p(s)-\mathcal{F}(0, s)
$$

where $p(t)$ is the FPTD and the kernel $K(x, s)=s x \Theta(x)-\left(\kappa|x|^{\mu-1}\right)$. This equation is formally of the Wiener-Hopf type of the first kind [Gakhov (1966)]. After some manipulations similar to those applied in reference [Zumofen and Klafter (1995)], we arrive at the asymptotic expression $p(s) \simeq 1-C s^{1 / 2}$, where $C=$ const, in accordance with the universal behaviour (57) and with the findings in reference [Zumofen and Klafter (1995)]. Thus, the dynamic equation (66) consistently phrases the FPTD problem for LFs. A numerical analysis of an LF in the presence of an absorbing boundary is shown in figure 18, nicely corroborating the Sparre-Andersen universality for various Lévy exponents [Chechkin et al (2003)]. In these simulations, a particle was removed when its jump would move it across the absorbing boundary. The simulations also demonstrate that the measured FPTD cannot be described by the other two (in this case inadequate) approaches, the images method and the direct definition addressed now.

This direct definition of the FPTD for Brownian processes uses the chain rule $\left(p_{\mathrm{fa}}(\tau)\right.$

$\ddagger$ It is only for processes, in which time $t$ and the number $n$ of steps are not a linear function of each other that the Sparre-Andersen universality is broken [Feller (1968), Redner (2001), Sparre Andersen (1953), Sparre Andersen (1954), Spitzer (1960)], for instance, for subdiffusion or LWs.

$\S$ This definition is thus significantly different from the Brownian case in which it does not matter whether the lower integration limit is 0 , or actually $-\infty$. 


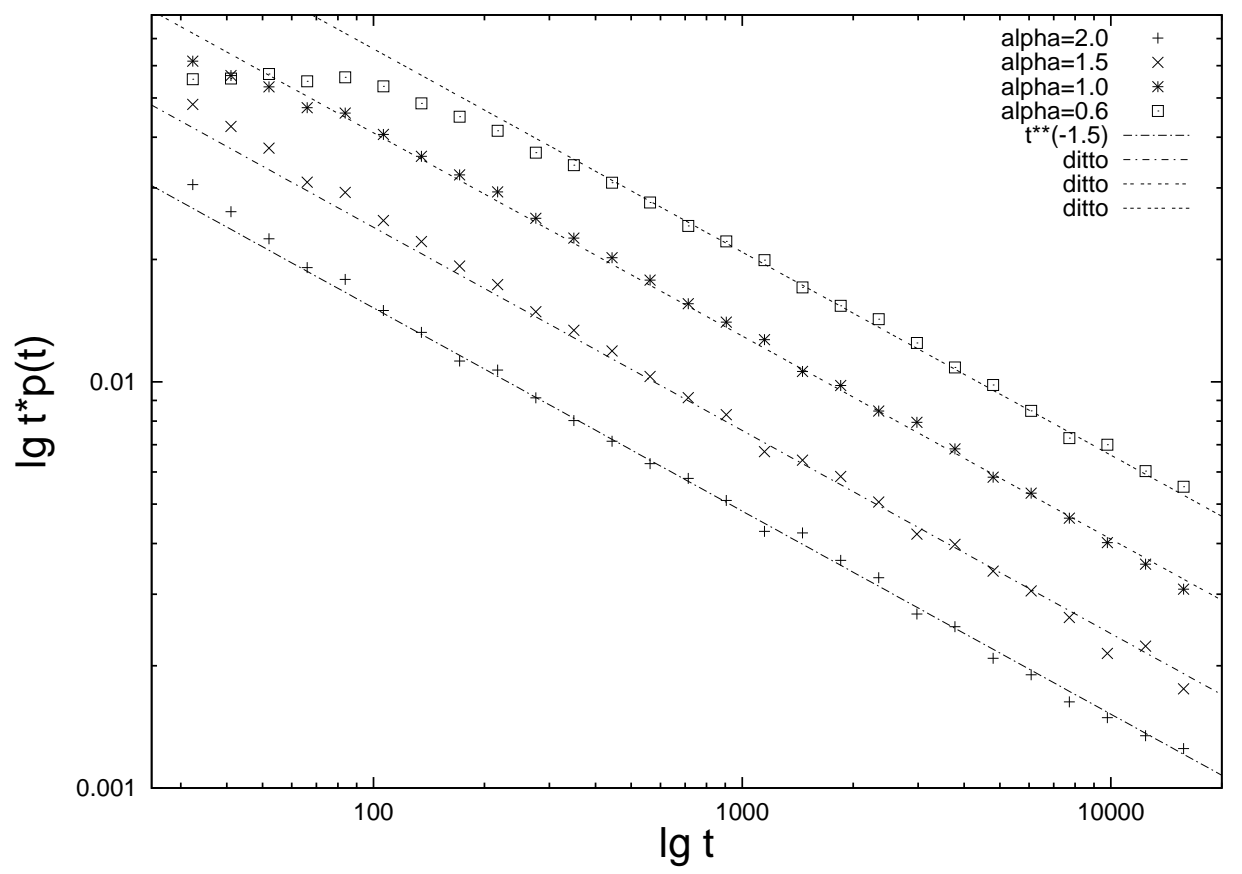

Figure 18. First passage time density for LFs with different index. The universal (Sparre Andersen) decay with power-law index $-3 / 2$ is nicely fulfilled.

depends implicitly on $x_{0}$ ) [Hughes (1995), Redner (2001)]

$$
P\left(-x_{0}, t\right)=\int_{0}^{t} p_{\mathrm{fa}}(\tau) P(0, t-\tau) .
$$

More precisely, this convolution, which corresponds to the algebraic relation $p_{\mathrm{fa}}(u)=$ $P\left(-x_{0}, u\right) / P(0, u)$ in Laplace space, is the PDF of first arrival. However, in Gaussian processes (Brownian diffusion, subdiffusion), due to the local jumps, both notions are equivalent. This is no longer true for an LF. Here, by long jumps the LF-particle can repeatedly hop across the point, until it will eventually land there. For an LF, the chain rule will therefore produce a too slowly decaying FPTD [Chechkin et al (2003)]. Equation (68) is equivalent to a process with a $\delta$-sink paraphrased by the dynamical equation

$$
\frac{\partial}{\partial t} f(x, t)=D \frac{\partial^{\mu}}{\partial|x|^{\mu}} f(x, t)-p_{\mathrm{fa}}(t) \delta(x),
$$

For an LF with index $\mu$, one would therefore expect the PDF of first arrival to decay like

$$
p_{\mathrm{fa}}(t) \sim t^{-2+1 / \mu} .
$$

This was investigated by numerical simulation, as shown in figure 19. A small interval $w$ around the sink was fixed, and any particle jumping into this zone would be removed.

We note that LWs on a finite domain and in the presence of absorbing boundary conditions was studied extensively in reference [Drysdale and Robinson (1998)]. A onesided Lévy stable jump length PDF was investigated in [Eliazar and Klafter (2004)]; 


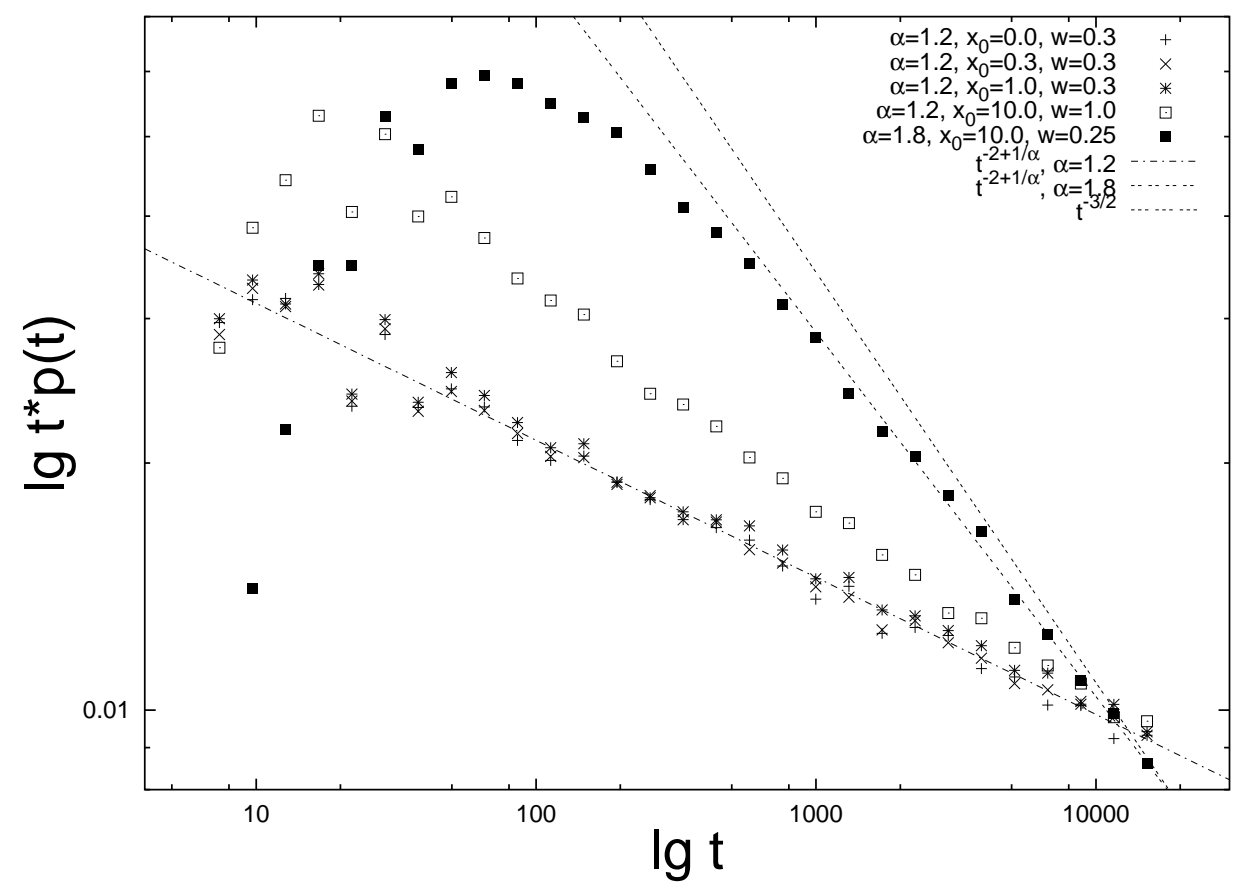

Figure 19. First arrival PDF for $\mu=1.2$ demonstrating the $t^{-2+1 / \mu}$ scaling, for optimal trap width $w=0.3$. For comparison, we show the same scaling for $\mu=1.8$, and the power-law $t^{-3 / 2}$ corresponding to the FPTD. The behaviour for too large $w=1.0$ shows a shift of the decay towards the $-3 / 2$ slope. Note that on the abscissa we plot $\lg t p(t)$. Note also that for the initial condition $x_{0}=0.0$, the trap becomes activated after the first step, consistent with [Zumofen and Klafter (1995)].

due to its asymmetric character, such a process is not subject to the SparreAndersen theorem. Indeed, the detailed analysis of this 'shot-noise' type process with one-sided, long-tailed $\lambda(x)$ for the special case $\mu=1 / 2$ reveals the FPTD $a\left(\pi x_{0}\right)^{-1 / 2} \exp \left(-a^{2} t^{2} /\left(4 x_{0}\right)\right)$ where $a$ is the amplitude of the Lévy stable law, and $x_{0}$ is the distance of the absorbing barrier from the initial location [Eliazar and Klafter (2004)].

\subsection{Reaction-diffusion processes}

The above result that for LFs the first arrival and the first passage are in fact different from each other is expected to have profound implications to diffusionreaction under Lévy jump length conditions. Similarly, subdiffusion conditions will change the dynamics of reactive systems. We note that the theoretical modelling of reaction-diffusion processes within a stochastic framework goes back to [von Smoluchowski (1916), von Smoluchowski (1916a)] in which the encounter of two independently diffusing particles is considered. The simplest version, diffusing particles in the presence of an immobile reaction centre $\mathrm{C}$, at which particles undergo an annihilation reaction $\mathrm{A}+\mathrm{C} \rightarrow 0$, is described by the first arrival time results obtained above: the decay of the concentration of $\mathrm{A}$ follows $\sim t^{-\alpha / 2}$ in subdiffusion with 
$0<\alpha<1$, and $\sim t^{-1+1 / \alpha}$ for an LF of Lévy index $1<\alpha<2$. Note that in the latter case, the reaction becomes successively stalled for $\alpha$ getting close to the Cauchy case $\alpha=1$, and that it approaches the Brownian case $\sim t^{-1 / 2}$ for $\alpha \rightarrow 2$.

In references [Yuste and Lindenberg (2001), Yuste and Lindenberg (2002)], the problem of subdiffusive reaction-diffusion of the coagulation type $A+A \rightarrow A$ was considered. Thus, the quantity of interest, the density function $E(x, t)$ to find an interval of length $x$ empty of any particle at time $t$, was shown to be described by the fractional diffusion equation

$$
\frac{\partial}{\partial t} E(x, t)=2{ }_{0} D_{t}^{1-\alpha} K_{\alpha} \frac{\partial^{2}}{\partial x^{2}} E(x, t)
$$

where we note the occurrence of the additional factor 2. For an initially random interparticle distribution $\lambda e^{-\lambda x}$ of the Poisson type, one can, for instance, obtain the concentration of particles,

$$
c(t)=\lambda E_{\alpha / 2}\left(-\lambda \sqrt{2 K_{\alpha}} t^{\alpha / 2}\right)
$$

which exhibits a temporal decay of the Mittag-Leffler kind, with scaling exponent $\alpha / 2$. Similarly, approximate expression for the time behaviour of the interparticle distance can be obtained. The analysis in reference [Yuste and Lindenberg (2001)] highlights the advantages of having at hand a formulation of the process in terms of a dynamical equation, i.e., the fractional diffusion equation. Compare the derivation in reference [Henry and Wearne (2000)].

A fractional diffusion equation for the geminate reaction of a particle $\mathrm{B}$, which starts a distance $r_{0}$ away from particle $\mathrm{A}$ is given as follows. B moves subdiffusively towards $\mathrm{A}$, until it reaches the encounter distance within the range $R, \ldots, R+d r$. This process was shown to be controlled by the fractional diffusion-reaction equation [Seki et al (2003), Seki et al (2003a)] (compare reference [Sung et al (2002)])

$$
\frac{\partial}{\partial t} C(r, t)={ }_{0} D_{t}^{1-\alpha}\left(K_{\alpha} \nabla^{2}-k_{\alpha} \frac{\delta(r-R)}{4 \pi R^{2}}\right) C(r, t)
$$

for the concentration $C(r, t)$, where we retained the spherical notation from reference [Seki et al (2003), Seki et al (2003a)]. Here, the generalised rate constant is $k_{\alpha}$.

A systematic derivation of reaction-diffusion equations with distributed delays (memory kernels), including their connection to fractional reaction-diffusion equations are discussed, and applied to the modelling of the (neolithic) transition from the hunting and gathering to the agricultural society in reference [Vlad and Ross (2002)]. In a two-species fractional reaction-diffusion system Turing instabilities were identified by [Henry and Wearne (2002)].

In reference [del-Castillo-Negrete et al (2003)] it is investigated the front dynamics in a reaction-diffusion system based on an asymmetric jump distribution, of Gaussian nature to the left, say, and Lévy stable to the right, related to the study of a birth-death process with similar jump rules [Sokolov and Belik (2003)]. In a similar study, superfast reaction front propagation was observed in the presence of long-tailed increments [Mancinelli et al (2002)], is based on a fractional version of the Fisher-Kolmogorov 

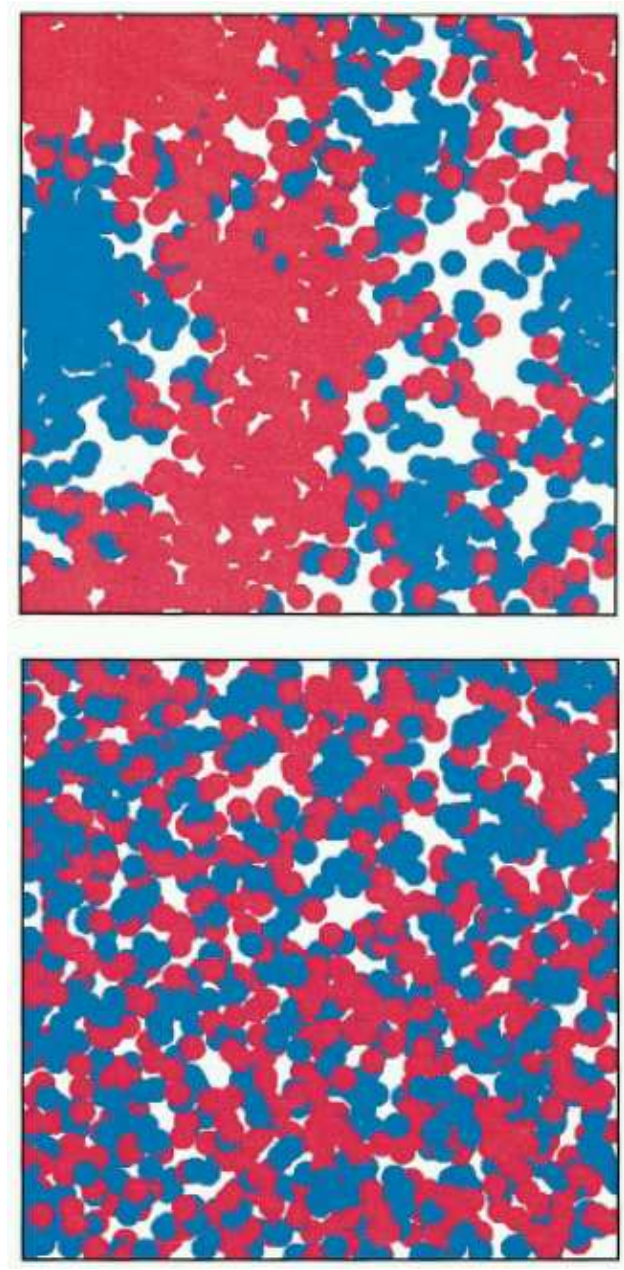

Figure 20. Top: Ovchinnikov-Zeldovich segregation for nearest-neighbour random walks under $\mathrm{A}+\mathrm{B} \rightarrow 0$ reaction conditions where the two colours symbolise the two reactant species. Bottom: The same process under Lévy mixing conditions, the Ovchinnikov-Zeldovich segregation is removed [Zumofen et al (1996a)].

equation. The efficiency of Lévy statistics in mixing of chemical reactions was shown in a numerical study of an $\mathrm{A}+\mathrm{B} \rightarrow 0$ reaction [Zumofen et al (1996a), Zumofen et al (1996)]. Accordingly, Lévy walks under certain parametric conditions can avoid the OvchinnikovZeldovich segregation of the reactants, which under inefficient mixing-conditions impedes speedy reactions, refer to figure 20. Finally, subdiffusive reaction dynamics was investigated in detail in [Blumen et al (1986a)].

\section{Summary}

Fractional dynamics ideas have been extensively explored during the recent few years, both theoretically and experimentally. It was therefore timely to update our review [Metzler and Klafter (2000)], in order to provide a basic reference for colleagues who are either actively working in the field of fractional dynamics, or who would like to 
get a quick overview. We decided to write this new review in a self-contained manner. In particular, we gathered a number of applications, and went more deeply into Lévy flight-type processes. Finally, we discussed in detail first passage and reaction-diffusion problems under anomalous conditions. In contrast, we did not repeat the historical and mathematical context from the earlier work [Metzler and Klafter (2000)], and we refer to that work for details, in particular, how to obtain explicit solutions in terms of Fox $H$-functions and via the method of separation of variables, and for earlier references to papers on fractional dynamics.

We concentrated on fractional integrodifferential operators of the RiemannLiouville type (acting on time $t$ ) and the Riesz-Weyl (position $x$ ) type. These follow straightforwardly from physical principles such as the continuous time random walk [Compte (1996), Metzler et al (1999a)], the generalised master equation [Metzler (2001)], the continuous time Chapman-Kolmogorov equation [Metzler (2000)], a multiple trapping model [Metzler and Klafter (2000a), Metzler and Klafter (2000b)], the Langevin equation with a Lévy noise source [Seshadri and West (1982), Fogedby (1994), Peseckis (1987), Schertzer et al (2001)], or subordination [Sokolov ()]. In particular, the equations obtained through these methods (probabilistic approach!) define a proper PDF as their solution, compare the discussion in references [Brockmann and Sokolov (2002), Sokolov (2002)]. There exist numerous other definitions [Samko et al (1993), Srivastava and Saxena (2001), Hilfer (2000)], whose potential relation to concrete physical systems has not yet been fully explored. We note that there exist other approaches to fractional equations, and connections to other physical processes. Thus, it was shown that the FFPE emerges as the master equation for a Langevin process the internal clock of which is the FPTD of a self-similar Markov process [Stanislavsky (2003)] (compare to the fractional Langevin equation [Lutz (2001a), Picozzi and West (2002)]). Similarly, a nonMarkovian stochastic Liouville equation was recently discussed [Shushin (2003)], and a statistical functional method to derive LFs was explored in [Vlad et al (2000)]. New aspects of the relation between deterministic systems and anomalous transport [Zaslavsky (1999), Zaslavsky (2002), Klafter and Zumofen (1994a)] have recently been discussed [Artuso and Cristadoro (2003)], also from a quantum perspective [Iomin and Zaslavsky (2002), Laskin (2002), Banerjee et al (2002)], and effects of memory in the sense of macroscopic time are discussed in [Zaburdaev (2003)]. LFs in quenched jump length fields are investigated in a renormalisation group approach in [Schulz (2002), Schulz and Reineker (2002)]. We also note that the potential conflict between PDF and trajectory description of anomalous transport was considered in [Bologna et al (2002)]. Finally, it should be mentioned that finite sampling effects for LFs were investigated in reference [Condat et al (2002)].

Apart from the solutions discussed herein, exact and approximate solutions of the FFPE were established in a double-well potential for rotational dipoles [Kalmykov et al (2003)], for the fractional oscillator [Ryabov and Puzenko (2002)], and for double-well anomalous diffusion in [Shushin (2001)]. Similarly, fixed axes 
dipoles rotating in an $N$-fold cosine potential were investigated on the basis of the fractional Klein-Kramers equation (14) in reference [Coffey et al (2003)]. The fractional Fokker-Planck equation on a comb structure was explored by [Zahran et al (2003), El-Wakil et al (2002)]. Experimentally, such predictions are being explored in reference [Jadżyn (2003)]. Cole-Cole type relaxation patterns were studied in dielectric systems [Ryabov et al (2003), Feldman et al (2002)], and applied to seismic wave attenuation [Hanyga (2003)]. 'Reverse engineering', i.e., the exploration of how to design a Lévy noise-driven Langevin system, which yields a given steady state behaviour, was studied in reference [Eliazar and Klafter (2003)]. Stationarity-conservation laws could be determined for fractional differential equations with variable coefficients [Klimek (2002)].

From the phase space or position space fractional-dynamical equations, one can by integration obtain the purely time-dependent relaxation behaviour of the system under consideration. Given the anomalous diffusion behaviour described by such dynamics, also the relaxation patterns will deviate from the classical exponential MaxwellDebye form [Plonka (2001), Ramakrishnan and Raj Lakshmi (1987)]. Fractional relaxation models have, inter alia, been discussed in references [Blumen et al (2002), Richert (2002), Schiessel et al (1995), Uchaikin (2003)]. Applications of such anomalous relaxation models have been impressively demonstrated over some 10 to 15 decades in frequency for dielectric relaxation [Hilfer (2003)] and polymeric systems [Glöckle and Nonnenmacher (1991), Metzler et al (1995)]. A comparative study of different anomalous relaxation models was undertaken in [Talkner (2001)]. Compare also to dynamical disorder models generalising the KWW-stretched exponential form [Vlad et al (1998), Vlad et al (1996)].

\section{Acknowledgments}

We acknowledge discussions with Aleksei Chechkin, Igor Sokolov, and Iddo Eliazar.

\section{Appendix A. Continuous time random walk approach to fractional dynamics}

A standard textbook random walk process is assumed to perform a step of fixed length in a random direction at each tick of a system clock, i.e., having constant spatial and temporal increments, $\Delta x$ and $\Delta t$. Such a process will give rise to the standard diffusion process in the long time limit, i.e., after a sufficient number of steps, and the associated random variable $x(t)=N^{-1 / 2} \sum_{i}^{N} x_{i}$, where $x_{i}$ is the position after the $i$ th step, will be distributed by a Gaussian due to the central limit theorem. A convenient generalisation of this process is the so-called continuous time random walk (CTRW) process, originally introduced by Weiss and Montroll [Montroll and Weiss (1965)], in which both jump length and and waiting time are distributed according to two PDFs, $\lambda(x)$ and $\psi(t)$. A CTRW therefore is based on a probabilistic concept. It is easy to show that the propagator for such a CTRW process in the absence of an external force is given in 
terms of the simple expression

$$
P(k, u)=\frac{1-\psi(u)}{u[1-\psi(k, u)]}
$$

in Fourier-Laplace space [Klafter et al (1987)]. A detailed review is the book by Hughes [Hughes (1995)]. We note that this framework is based on a Euclidean space, and has to be modified for transport on supports with fractal dimension [Ben-Avraham and Havlin (2000), Metzler et al (1994), Metzler and Nonnenmacher (1997), Kobelev et al (2003), Kobelev et al (2002), Ren et al (2003), Ren et al (2003a), Acedo and Yuste (1998)].

\section{The waiting time and jump length PDFs}

A waiting time PDF of the long-tailed inverse power-law form $\psi(t) \sim \tau^{\alpha} / t^{1+\alpha}$ $(0<\alpha<1)$, which enters the CTRW propagator (A.1) via the Laplace expansion $\psi(u) \sim 1-(u \tau)^{\alpha}(u \ll \tau)$, can be completed, for instance, to a one-sided Lévy stable density $L_{\alpha, \tau}^{+}(t)$ whose characteristic function in Laplace space is $\varphi(u)=$ $\int_{0}^{\infty} L_{\alpha, \tau}^{+}(t) e^{-u t} d t=\exp \left(-[u \tau]^{\alpha}\right) \quad$ [Lévy (1954), Gnedenko and Kolmogorov (1954), Hughes (1995), Samorodnitsky and Taqqu (1994), Re et al (2003)]. This complete, explicit representation for $\psi(t)$ has the advantage that it includes the limit $\alpha=1$, in which case $\psi(u)=e^{-u \tau} \sim 1-u \tau$, and $\psi(t)=\delta(t-\tau)$. Another possibility is to choose $\psi(t)=\left(\tau^{\alpha} / t^{1-\alpha}\right) E_{\alpha, \alpha}\left(-[t / \tau]^{\alpha}\right)$, where $E_{\alpha, \alpha}$ is the generalised Mittag-Leffler function with the series expansion $\psi(t)=\left(\tau^{\alpha} / t^{1-\alpha}\right) \sum_{n=0}^{\infty}\left(-[t / \tau]^{\alpha}\right) / \Gamma(\alpha n+\alpha)$, as derived by [Hilfer and Anton (1995)]. In this case, the limit $\alpha=1$ becomes $\psi(t)=e^{-t / \tau}$.

Similarly, the jump length $\operatorname{PDF} \lambda(x)$ can be assumed to be given by a symmetric Lévy stable PDF defined in terms of its characteristic function, $\varphi(k) \equiv$ $\int_{-\infty}^{\infty} \lambda(x) \exp (\mathrm{i} k x) d x=\exp \left(-[\sigma|k|]^{\mu}\right)$, where $0<\mu \leq 2$. In the case $\mu=2$, we obviously recover the Gaussian distribution, which corresponds to a standard jump length distribution. For $0<\mu<2, \lambda(x) \sim \sigma^{\mu} /|x|^{1+\mu}$, and in particular, its variance diverges [Hughes (1995), Lévy (1954)].

\section{References}

[Abramowitz and Stegun (1972)]Abramowitz M and Stegun I 1972 Handbook of Mathematical Functions (Dover, New York)

[Acedo and Yuste (1998)]Acedo L and Yuste SB 1998 Phys. Rev. E 575160

[Adams and Gelhar (1992)]Adams EE and Gelhar LW 1992 Wat. Res. Res. 283293

[Allegrini et al (2003)]Allegrini P, Aquino G, Grigolini P, Palatella L and Rosa A 2003 Phys. Rev. E 68056123

[Altan-Bonnet et al (2003)]Altan-Bonnet G, Libchaber A and Krichevsky O 2003 Phys. Rev. Lett. 90 138101

[Amblard et al (1996)]Amblard F, Maggs AC, Yurke B, Pargellis AN and Leibler S 1996 Phys. Rev. Lett. 774470 [Erratum 1998 ibid. 81 1136; Reply 1998 ibid. 81 1135]

[Artuso and Cristadoro (2003)]Artuso R and Cristadoro G 2003 Phys. Rev. Lett. 90244101

[Atkinson et al (2002)]Atkinson RPD, Rhodes CJ, Macdonald DW, Anderson RM 2002 OIKOS 98134 
[Austin et al (1974)]Austin RH, Beeson K, Eisenstein L, Frauenfelder H, Gunsalus IC and Marshall VP 1974 Phys. Rev. Lett. 32403

[Austin et al (1975)]Austin RH, Beeson K, Eisenstein L, Frauenfelder H and Gunsalus IC 1975 Biochem. 145355

[Baeumer et al (2003)]Baeumer B, Benson DA and Meerschaert MM 2003 Advection and dispersion in time and space Math. Models and Methods in Applied Sciences, at press

[Bakk and Metzler (2004)]Bakk A and Metzler R 2004 In vivo nonspecific binding of $\lambda$ CI and Cro repressors is significant Submitted

[Bakk and Metzler (2004a)]Bakk A and Metzler R 2004a Nonspecific binding of the $O_{R}$ repressors $C I$ and Cro of bacteriophage $\lambda$ Submitted

[Bakunin (2003)]Bakunin OG 2003 Plasma Phys. Rep. 29955

[Balakrishnan (1985)]Balakrishnan V 1985 Physica A 132569

[Banerjee et al (2002)]Banerjee D, Banik SK, Bag BC, Ray DS 2002 Phys. Rev. E 66051105

[Bao (2003)]Bao JD 2003 Phys. Lett. A 314203

[Bao and Zhuo (2003)]Bao J-D and Zhuo Y-Z 2003 Phys. Rev. C 67064606

[Bardou et al (2002)]Bardou F, Bouchaud J-F, Aspect A and Cohen-Tannoudji C 2002 Lévy statistics and laser cooling (Cambridge University Press, Cambridge)

[Barkai and Silbey (1999)]Barkai E and Silbey R 1999 Chem. Phys. Lett. 310287

[Barkai and Silbey (2000)]Barkai E and Silbey R 2000 J. Phys. Chem. B 1043866

[Barkai et al (2000)]Barkai E, Silbey R and Zumofen G 2000 Phys. Rev. Lett. 845339

[Barkai et al (2000a)]Barkai E, Metzler R and Klafter J 2000a Phys. Rev. E 61132

[Barkai (2001)]Barkai E 2001 Phys. Rev. E 63046118

[Barkai (2002)]Barkai E 2002 Chem. Phys. 28413

[Barkai (2003)]Barkai E 2003 Phys. Rev. E 68055104

[Barkai (2003a)]Barkai E 2003 Phys. Rev. Lett. 90104101

[Barkai and Cheng (2003)]Barkai E and Cheng YC 2003 J. Chem. Phys. 1186167

[Barkai et al (2003)]Barkai E, Naumov AV, Vainer YuG, Bauer M and Kador L 2003 Lévy statistics for random single-molecule line shapes in glasses Eprint cond-mat/0307257

[Barsegov and Mukamel (2004)]Barsegov V and Mukamel S 2004 J. Phys. Chem. 10815

[Baskin and Zilberstein (2002)]Baskin E and Zilberstein G 2002 Electrophoresis 232636

[Bates et al (2003)]Bates M, Burns M and Meller A 2003 Biophys. J. 842366

[Bazzani et al (2003)]Bazzani A, Bassi G and Turchetti G 2003 Physica A 324530

[Ben-Avraham and Havlin (2000)]Ben-Avraham D and Havlin S 2000 Diffusion and Reactions in Fractals and Disordered Systems (Cambridge University Press, Cambridge)

[Ben-Yacov (2002)]Segev R, Benveniste M, Hulata E, Cohen N, Palevski A, Kapon E, Shapira Y and Ben-Jacob E 2002 Phys. Rev. Lett. 88118102

[Benson et al (2001)]Benson DA, Schumer R, Meerschart MM and Wheatcraft SW 2001 Transp. Porous Media 42211

[Berg et al (1981)]Berg OG, Winter RB and von Hippel PH 1981 Biochem. 206929

[Berkowitz and Scher (1995)]Berkowitz B and Scher H 1995 Wat. Res. Res. 311461

[Berkowitz and Scher (1997)]Berkowitz B and Scher H 1997 Phys. Rev. Lett. 794038

[Berkowitz et al (2000)]Berkowitz B, Scher H and Silliman SE 2000 Wat. Res. Res. 36149 [Correction 2000 ibid. 36 1371]

[Berkowitz et al (2002)]Berkowitz B, Klafter J, Metzler R and Scher H 2002 Wat. Res. Res. 381191

[Bisquert (2003)]Bisquert J 2003 Phys. Rev. Lett. 91010602

[Bisquert et al (2003)]Bisquert J, Garcia-Belmonte G and Pitarch Á 2003 Chem. Phys. Phys. Chem. 3 287

[Blatz and Magleby (1986)]Blatz AL and Magleby KL 1986 J. Physiol. (London) 378141

[Blumen et al (1986)]Blumen A, Klafter J and Zumofen G 1986 In Optical Spectroscopy of Glasses edited by Zschokke I (Reidel, Dordrecht)

[Blumen et al (1986a)]Blumen A, Klafter J and Zumofen G 1986a In Fractals in Physics edited by 
Pietronero L (North Holland, Amsterdam)

[Blumen et al (2002)]Blumen A, Gurtovenko AA and Jespersen S 2002 J. Non-cryst. Sol. 30571

[Boggs et al (1993)]Boggs JM, Beard LM, Long SE and McGee MP 1993 Database for the second macrodispersion experiment (MADE-2), EPRI report TR-102072. (Palo Alto, CA, US: Electric Power Res. Inst.)

[Boldyrev and Gwinn (2003)]Boldyrev S and Gwinn CR 2003 Phys. Rev. Lett. 91131101

[Bologna et al (2002)]Bologna M, Grigolini P and West BJ 2002 Chem. Phys. 284115

[Borland (1998)]Borland L 1998 Phys. Rev. E 576634

[Bouchaud and Georges (1990)]Bouchaud JP and Georges A 1990 Phys. Rep. 195127

[Bouchaud and Potters (2000)]Bouchaud J-P, Potters M. 2000. Theory of financial risks. (Cambridge: Cambridge University Press)

[Brockmann and Sokolov (2002)]Brockmann D and Sokolov IM 2002 Chem. Phys. 284409

[Brockmann and Geisel (2003)]Brockmann D and Geisel T 2003 Phys. Rev. Lett. 90170601

[Brockmann and Geisel (2003a)]Brockmann D and Geisel T 2003a Phys. Rev. Lett. 91048303

[Buldyrev et al (2001)]Buldyrev SV, Gitterman M, Havlin S, Kazakov AY, da Luz MGE, Raposo EP, Stanley HE and Viswanathan GM 2001 Physica A 302148

[Caputo (1969)]Caputo M 1969 Elasticità e dissipazione (Zanichelli, Bologna)

[Caputo (2001)]Caputo M 2001 Fract. Calc. Appl. Anal. 4421

[Carati et al (2003)]Carato A, Galgani L and Pozzi B 2003 Phys. Rev. Lett. 90010601

[Caspi et al (2000)]Caspi A, Granek R and Elbaum M 2000 Phys. Rev. Lett. 855655

[Caspi et al (2001)]Caspi A, Yeger O, Grosheva I, Bershadsky AD and Elbaum M 2001 Biophys. J. 81 1990

[Caspi et al (2002)]Caspi A, Granek R and Elbaum M 2002 Phys. Rev. E 66011916

[Chandrasekhar (1943)]Chandrasekhar S 1943 Rev. Mod. Phys. 151

[Chechkin et al (2002)]Chechkin A, Gonchar V, Klafter J, Metzler R and Tanatarov L 2002 Chem. Phys. 284233

[Chechkin et al (2002a)]Chechkin AV, Gonchar VY and Szydlowsky M 2002a Phys. Plasma 978

[Chechkin et al (2002b)]Chechkin AV, Gorenflo R and Sokolov IM 2002 Phys. Rev. E 66046129

[Chechkin et al (2003)]Chechkin AV, Metzler R, Gonchar VY, Klafter J andT anatarov LV $2003 \mathrm{~J}$. Phys. A $36 \mathrm{~L} 537$

[Chechkin et al (2003a)]Chechkin AV, Gonchar VY, Klafter J, Metzler R and Tanatarov LV. 2003. Lévy flights in a steep potential well: finite variance and dynamical turnover between states of different modality. Eprint cond-mat/0306601 J. Stat. Phys. accepted

[Chechkin et al (2003b)]Chechkin AV, Klafter J, Gonchar VY, Metzler R and Tanatarov LV 2003 Phys. Rev. E 67 010102(R)

[Chechkin et al (2003c)]Chechkin AV, Klafter J and Sokolov IM 2003 Europhys. Lett. 63326

[Chechkin et al (2004)]Chechkin AV, Metzler R, Gonchar VY, Klafter J, Tanatarov LV. 2004 Normalising Lévy flights by dissipative non-lineratity Unpublished

[Chuang et al (2002)]Chuang J, Kantor Y and Kardar M 2002 Phys. Rev. E 65011802

[Coffey et al (2003)]Coffey WT, Kalmykov YP and Titov SV 2003 Phys. Rev. E 67061115

[Compte (1996)]Compte A 1996 Phys. Rev. E 534191

[Compte and Metzler (1997)]Compte A and Metzler R 1997 J. Phys. A 307277

[Condat et al (2002)]Condat CA, Rangel J and Lamberti PW 2002 Phys. Rev. E 65026138

[Costa et al (2003)]Costa IVL, Morgado R, Lima MVBT and Oliveira FA 2003 Europhys. Lett. 63173

[de Almeida and Baumvoll (2003)]de Almeida RMC and Baumvol IJR 2003 Surf. Sci. Rep. 491

[Déjardin (2003)]Déjardin JL 2003 Phys. Rev. E 68031108

[del-Castillo-Negrete et al (2003)]del-Castillo-Negrete D, Carreras BA and Lynch VE 2003 Phys. Rev. Lett. 91018302

[Denisov et al (2003)]Denisov S, Klafter J and Urbakh M 2003 Phys. Rev. Lett. 91194301

[Dickman (2003)]Dickman R 2003 Phys. Rev. Lett. 90108701

[Dräger and Klafter (2000)]Dräger J and Klafter J 2000 Phys. Rev. Lett. 845998 
[Drysdale and Robinson (1998)]Drysdale PM and Robinson PA 1998 Phys. Rev. E 585382

[Dworecki et al (2003)]Dworecki K, Wạsik S and Kosztołowicz T 2003 Acta Physica Polonica B 34 3695

[Eliazar and Klafter (2004)]Eliazar I and Klafter J 2004 Physica A 336, 219

[Eliazar and Klafter (2003)]Eliazar I and Klafter J 2003 J. Stat. Phys. 111739

[El-Wakil et al (2002)]El-Wakil SA, Zahran MA and Abulwafa EM 2002 Physica A 30327

[Erdélyi (1954)]Erdélyi A (Ed.) 1954 Tables of Integral Transforms (Bateman Manuscript Project Vol. I) (New York: McGraw-Hill)

[Fa (2003)]Fa KS 2003 Chem. Phys 2871

[Fa and Lenzi (2003)]Fa KS and Lenzi EK 2003 Phys. Rev. E 67061105

[Falla et al (2004)]Falla R, Grigolini P, Ignaccolo M and Schwettmann A 2004 The random growth of interfaces as a subordinated process Preprint

[Feder (1988)]Feder J 1988 Fractals (New York: Plenum Press)

[Feldman et al (2002)]Feldman Y, Puzenko A and Ryabov Y 2002 Chem. Phys. 284139

[Feller (1968)]Feller W 1968 An Introduction to Probability Theory and Its Applications (John Wiley \& Sons/New York)

[Feodotov and Mendez (2000)]Feodotov S and Mendez V 2002 Phys. Rev. E 66030102

[Feynman and Vernon (1963)]Feynman RP and Vernon FL 1963 Ann. Phys. (N.Y.) 24118

[Fischer et al (1996)]Fischer E, Kimmich R and Fatkullin N 1996 J. Chem. Phys. 1049174

[Flomenbom and Klafter (2003)]Flomenbom O and Klafter J 2003 Phys. Rev. E 68041910

[Flomenbom and Klafter (2004)]Flomenbom O and Klafter J 2004 Biophys. J. at press

[Fogedby (1994)]Fogedby HC 1994 Phys. Rev. Lett. 732517

[Fogedby (1994a)]Fogedby HC 1994a Phys. Rev. E 501657

[Fogedby (1998)]Fogedby HC 1998 Phys. Rev. E 581690

[Friedrich (2003)]Friedrich R 2003 Phys. Rev. Lett. 90084501

[Frisch and Frisch (1995)]Frisch U and Frisch H 1995 In Lévy flights and related topics in physics Edited by Shlesinger MF, Zaslavsky GM and Frisch U (Berlin:Springer-Verlag)

[Gakhov (1966)]Gakhov FD 1966 Boundary value problems (Pergamon Press, Oxford)

[García-García (2003)]García-García AM 2003 Classical intermittency and quantum Anderson transition Eprint cond-mat/0309445

[Gelhar et al (1992)]Gelhar LW, Welty C and Rehfeldt KR 1992 Wat. Res. Res. 281955

[Gerstner and Kistler (2002)] Gerstner W and Kistler WM 2002 Spiking Neuron Models. (Cambridge: Cambridge University Press)

[Gitterman (2000)]Gitterman M 2000 Phys. Rev. E 626065

[Glöckle and Nonnenmacher (1991)]Glöckle WG and Nonnenmacher TF 1991 Macromol. 246426

[Glöckle and Nonnenmacher (1994)]Glöckle WG and Nonnenmacher TF 1994 Rheol. Acta 33337

[Glöckle and Nonnenmacher (1995)]Glöckle WG and Nonnenmacher TF 1995 Biophys. J. 6846

[Gnedenko and Kolmogorov (1954)]Gnedenko BV and Kolmogorov AN 1954 Limit Distributions for Sums of Random Variables (Reading, MA: Addison-Wesley)

[Goldar et al (2001)]Goldar A, Roser SJ, Caruana D, Gerstenberg MC, Hughes AV and Edler KJ 2001 Phys. Chem. Chem. Phys. 35559

[Gonchar et al (2003)]Gonchar VY, Chechkin AV, Sorokovoi EL, Chechkin VV, Grigor'eva LI and Volkov ED 2003 Plasma Phys. Rep. 29380

[Gorenflo (1997)]Gorenflo R 1997 In Fractals and fractional calculus in continuum mechanics Edited by Carpinteri A and Mainardi F (Wien:Springer)

[Gorenflo et al (2002)]Gorenflo R, Mainardi F, Moretti D, Pagnini G and Paradisi P 2002 Chem. Phys. $\mathbf{2 8 4} 521$

[Gorenflo et al (2002a)]Gorenflo R, Mainardi F, Moretti D, Pagnini G and Paradisi P 2002a Physica A 305106

[Granek and Klafter (2001)]Granek R and Klafter J 2001 Europhys. Lett. 5615

[Greco et al (2003)] Greco A, Taktakishvili AL, Zimbardo G, Veltri P, Cimino G, Zelenyi LM and Lopez 
RE 2003 J. Geophys. Res.-Space Phys. 1081395

[Hack (1957)]Hack JT 1957 U. S. Geol. Surv. Prof. Pap. B 29445

[Hanke and Metzler (2003)]Hanke A and Metzler R 2003 J. Phys. A 36 L473

[Hanyga (2003)]Hanyga A 2003 J. Comput. Acoust. 1175

[Havlin and Weiss (1990)]Havlin S and Weiss GH 1990 J. Stat. Phys. 581267

[Helmstetter and Sornette (2002)]Helmstetter A and Sornette D 2003 Phys. Rev. E 66061104

[Henry and Wearne (2000)]Henry BI and Wearne SL 2000 Physica A 276448

[Henry and Wearne (2002)]Henry BI and Wearne SL 2002 SIAM J. Appl. Math. 62870

[Hilfer (1995)]Hilfer R 1995 Fractals 3211

[Hilfer and Anton (1995)]Hilfer R and Anton L 1995 Phys. Rev. E 51 R848

[Hilfer (2000)]Hilfer R (Editor) 2000 Applications of fractional calculus in physics (Singapore: World Scientific)

[Hilfer (2002)]Hilfer R 2002 Chem. Phys. 284399

[Hilfer (2002a)]Hilfer R 2002 Phys. Rev. E 65061510

[Hilfer (2003)]Hilfer R 2003 Fractals 11251

[Honkonen (1996)]Honkonen J 1996 Phys. Rev. E 53327

[Hughes (1995)]Hughes BD 1995 Random Walks and Random Environments, Volume 1: Random Walks (Oxford: Oxford University Press)

[Hughes (2002)]Hughes BD 2002 Phys. Rev. E 65035105

[Hurst (1951)]Hurst HE 1951 Trans. Am. Soc. Civ. Eng. 116770

[Hurst et al (1951)]Hurst HE, Black RP and Simaika YM 1951 Long-term storage: An experimental study (London: Constable)

[Hwa et al (2003)]Hwa, T, Marinari E, Sneppen K, and Tang LH 2003 Proc. Natl. Acad. USA 1004411

[Iben et al (1989)]Iben IET, Braunstein D, Doster W, Frauenfelder H, Hong MK, Johnson JB, Luck S, Ormos P, Schulte A, Steinbach PJ, Xie AH and Young RD 1989 Phys. Rev. Lett. 621916

[Igloi et al (1999)]Igloi F, Turban L and Rieger H 1999 Phys. Rev. E 591465

[Iomin and Zaslavsky (2002)]Iomin A and Zaslavsky GM 2002 Chem. Phys. 2843

[Jadżyn (2003)]Jadżyn J, Czechowski G, Legrand C and Douali R 2003 Phys. Rev. E 67041705

[Jensen et al (2003)]Jensen MH, Johansen A and Simonsen I 2003 Physica A 324338

[Jespersen et al (1999)]Jespersen S, Metzler R and Fogedby HC 1999 Phys. Rev. E 592736

[Kalmykov et al (2003)]Kalmykov YP, Coffey WT and Titov SV 2003 Bimodal approximation for anomalous diffusion in a potential Preprint

[Katori et al (1997)]Katori H, Schlipf S and Walther H 1997 Phys. Rev. Lett. 792221

[Katzav (2003)]Katzav E 2003 Phys. Rev. E 68031607

[Kenty (1932)]Kenty C 1932 Phys. Rev. 42823

[Kim and Yoon (2003)]Kim K and Yoon SM 2003 Fractals 11131

[Kimmich (1997)]Kimmich R 1997 NMR: Tomography, Diffusometry, Relaxometry (Springer-Verlag, Berlin)

[Kimmich (2002)]Kimmich R 2002 Chem. Phys. 284253

[Kirchner et al (2000)]Kirchner JW, Feng X and Neal C 2000 Nature 403524

[Klafter et al (1987)]Klafter J, Blumen A and Shlesinger MF 1987 Phys. Rev. A 353081

[Klafter and Zumofen (1994)]Klafter J and Zumofen G 1994 J. Phys. Chem. 987366

[Klafter and Zumofen (1994a)]Klafter J and Zumofen G 1994 Phys. Rev. E 494873

[Klafter et al (1996)]Klafter J, Shlesinger MF and Zumofen G 1996 Physics Today 49(2) 33

[Klemm et al (2002)]Klemm A, Metzler R and Kimmich R 2002 Phys. Rev. E 65021112

[Klimek (2002)]Klimek M 2002 J. Phys. A 356675

[Klimontovich (1991)]Klimontovich YL 1991 Turbulent Motion and the Structure of Chaos (Kluwer, Dordrecht)

[Kobelev et al (2002)]Kobelev YL, Kobelev LY, Kobelev VL and Romanov EP 2002 Dokl. Phys. 47 580

[Kobelev et al (2003)]Kobelev YL, Kobelev LY and Klimontovich YL 2003 Dokl. Phys. 48264 
[Kolmogorov (1940)]Komlogorov AN 1940 Reports Acad. Sci. USSR (Doklady Akademii Nauk SSSR) 266

[Kondrashin et al (2002)]Kondrashin MP, Schaufler S, Schleich WP and Yakovlev VP 2002 Chem. Phys. 284319

[Köpf et al (1996)]Köpf M, Corinth C, Haferkamp O and Nonnenmacher TF 1996 Biophys. J. 702950

[Köpf et al (1998)]Köpf M, Metzler R, Haferkamp O and Nonnenmacher TF 1998 In Fractals in Biology and Medicine Edited by Losa GA, Merlini D, Nonnenmacher TF and Weibel ER (Basel: Birkhäuser)

[Kosztołowicz and Dworecki (2003)] Kosztołowicz T and Dworecki K 2003 Acta Physica Polonica B 34 3699

[Kubo et al (1985)]Kubo R, Toda M and Hashitsume N 1985 Statistical Physics II Solid State Sciences Vol 31 (Springer-Verlag, Berlin)

[Kusnezov et al (1999)]Kusnezov, D, Bulgac A and Dang GD 1999 Phys. Rev. Lett. 821136

[Lagutin and Uchaikin (2003)]Lagutin AA and Uchaikin VV 2003 Nucl. Instrum. Meth. B 201212

[Landau and Lifshitz (1984)]Landau LD, Lifshitz EM and Pitaevskii LP 1984 Electrodynamics of Continuous Media, 2nd edition (Oxford: Butterworth-Heinemann)

[Landau and Lifshitz (1987)]Landau LD and Lifshitz EM 1987 Fluid mechanics (Pergamon, Oxford)

[Langevin (1908)]Langevin P 1908 Comptes Rendus 146530

[Laskin (2002)]Laskin N 2002 Phys. Rev. E 66056108

[LeBlanc et al (1991)]LeBlanc DR, Garabedian SP, Hess KM, Gelhar LW, Quadri RD, Stollenwerk KG and Wood WW 1991 Wat. Res. Res. 27895

[LeGoff et al (2002)]LeGoff L, Hallatschek O, Frey E and Amblard F 2002 Phys. Rev. Lett. 89258101

[Leith (2003)]Leith JR 2003 Signal Processing 832397

[Lenzi et al (2003)]Lenzi EK, Mendes RS, Fa KS, Malacarne LC and da Silva LR 2003 J. Math. Phys. 442179

[Lenzi et al (2003a)]Lenzi EK, Mendes RS and Tsallis C 2003a Phys. Rev. E 67031104

[Levandowsky et al (1997)]Levandowsky M, White BS and Schuster FL 1997 Acta Protozool. 36237

[Lévy (1954)]Lévy P. 1954. Théorie de l'addition des variables aléatoires. Paris: Gauthier-Villars

[Lévy (1965)]Lévy P 1965 Processus stochastiques et mouvement Brownien (Paris: Gauthier-Villars)

[Lévy (1953)]Lévy P 1953 Univ. Californ. Publ. Statist. 1331

[Li and Wang (2003)]Li B and Wang J 2003 Phys. Rev. Lett. 91044301

[Lim and Muniandy (2002)]Lim SC and Muniandy SV 2002 Phys. Rev. E 66021114

[Lunkenheimer and Loidl (2002)]Lunkenheimer P and Loidl A Chem. Phys. 284205

[Luchko et al (1998)]Luchko Y and Gorenflo R 1998 Fract. Calc. Appl. Anal. 163

[Lugomer et al (2002)]Lugomer S, Maksimovic A and Stipancic M 2002 Phys. Scripta 65447

[Lutz (2003)]Lutz E 2003 Phys. Rev. A 67051402

[Lutz (2001)]Lutz E 2001 Phys. Rev. Lett. 862208

[Lutz (2001a)]Lutz E. 2001 Phys. Rev. E 64051106

[Lynch et al (2003)]Lynch VE, Carreras BA, Del-Castillo-Negrete D, Ferreira-Mejias KM and Hicks HR 2003 J. Comput. Phys. 192406

[Mainardi et al (2000)]Mainardi F, Raberto M, Gorenflo R and Scalas E 2000 Physica A 287468

[Mainardi et al (2001)]Mainardi F, Luchko Yu and Pagnini G 2001 Fract. Calc. Appl. Anal. 4153

[Mancinelli et al (2002)]Mancinelli R, Vergni D and Vulpiani A 2002 Europhys. Lett. 60532

[Mandelbrot (1963)]Mandelbrot BB 1963 J. Bus. 36394

[Mandelbrot (1966)]Mandelbrot BB 1966 J. Bus. 39242

[Mandelbrot (1967)]Mandelbrot BB 1967 Science 155636

[Mandelbrot (1967a)]Mandelbrot BB 1967 J. Bus. 40393

[Mandelbrot (1982)] Mandelbrot BB 1982 The fractal geometry of nature (Freeman, San Francisco)

[Mandelbrot and van Ness (1968)]Mandelbrot BB and van Ness JW 1968 SIAM Rev. 1422

[Mann and Woyczynski (2001)]Mann JA and Woyczynski WA 2001 Physica A 291159

[Mantegna and Stanley (1996)]Mantegna RN and Stanley HE 1996 Nature 383587

[Mantegna and Stanley (2000)]Mantegna RN and Stanley HE 2000. An introduction to econophysics: 
correlations and complexity in finance (Cambridge: Cambridge University Press)

[Mathai and Saxena (1978)] Mathai AM and Saxena RK 1978 The H-function with Applications in Statistics and Other Disciplines (New Delhi: Wiley Eastern Ltd)

[Matheron and de Marsily (1980)]Matheron G and de Marsily G 1980 Wat. Res. Res. 16901

[Matsumoto et al (2002)]Matsumoto M, Saito S and Ohmine I 2002 Nature 416409

[Meerschaert et al (2002)]Meerschaert MM, Benson DA, Scheffler H-P and Becker-Kern P 2002 Phys. Rev. E $66060102(\mathrm{R})$

[Meller (2003)]Meller A 2003 J. Phys. Cond. Matt. 15 R581

[Metzler et al (1994)]Metzler R, Glöckle WG and Nonnenmacher TF 1994 Physica A 21113

[Metzler et al (1995)]Metzler R, Schick W, Kilian HG and Nonnenmacher TF 1995 J. Chem. Phys. 1037180

[Metzler et al (1998)]Metzler R, Klafter J and Sokolov IM 1998 Phys. Rev. E 581621

[Metzler and Nonnenmacher (1998)]Metzler R and Nonnenmacher TF 1998 Phys. Rev. E 576409

[Metzler and Compte (1999)]Metzler R and Compte A 1999 Physica A 268454

[Metzler (2000)]Metzler R 2000 Phys. Rev. E 626233

[Metzler and Compte (2000)]Metzler R and Compte A 2000 J. Phys. Chem. B 1043858

[Metzler (2001)]Metzler R 2001 Euro. Phys. J. B 19249

[Metzler (2001a)]Metzler R 2001a Phys. Rev. E 63012103

[Metzler et al (1999)]Metzler R, Barkai E and Klafter J 1999 Phys. Rev. Lett. 823563

[Metzler et al (1999a)]Metzler R, Barkai E and Klafter J 1999a Europhys. Lett. 46431

[Metzler and Klafter (2000)]Metzler R and Klafter J 2000 Phys. Rep. 3391

[Metzler and Klafter (2000a)]Metzler R and Klafter J 2000a Phys. Rev. E 616308

[Metzler and Klafter (2000b)]Metzler R and Klafter J 2000b J. Phys. Chem. B 1043851

[Metzler and Klafter (2000c)]Metzler R and Klafter J 2000c Europhys. Lett. 51492

[Metzler and Klafter (2000d)]Metzler R and Klafter J 2000d Physica A 278107

[Metzler and Klafter (2001)]Metzler R and Klafter J 2001 Adv. Chem. Phys. 116223

[Metzler and Klafter (2000e)]Metzler R and Klafter J 2000e Chem. Phys. Lett. 321238

[Metzler and Klafter (2002)]Metzler R and Klafter J 2002 J. Non-Cryst. Solids 30581

[Metzler and Klafter (2003)]Metzler R and Klafter J 2003 Biophys. J. 852776

[Metzler and Nonnenmacher (2002)] Metzler R and Nonnenmacher TF 2002 Chem. Phys. 28467

[Metzler and Sokolov (2002)]Metzler R and Sokolov IM 2002 Europhys. Lett. 58482

[Metzler and Sokolov (2004)]Metzler R and Sokolov IM 2004 Phys. Rev. Lett. at press

[Metzler and Nonnenmacher (1997)]Metzler R and Nonnenmacher TF 1997 J. Phys. A 301089

[Milovanov and Zelenyi (2001)]Milovanov AV and Zelenyi LM 2001 Phys. Rev. E 64052101

[Milovanov and Zelenyi (2002)]Milovanov AV and Zelenyi LM 2002 Adv. Space Res. 302667

[Milovanov and Rasmussen (2002)]Milovanov AV and Rasmussen JJ 2002 Phys. Rev. B 66134505

[Miller and Ross (1993)]Miller KS and Ross B. 1993 An introduction to the fractional calculus and fractional differential equations (New York: Wiley)

[Mittag-Leffler (1903)]Mittag-Leffler GM 1903 C. R. Acad. Sci. Paris 137554

[Mittag-Leffler (1904)]Mittag-Leffler GM 1904 R. Acad. dei Lincei, Rendiconti 133

[Mittag-Leffler (1905)]Mittag-Leffler GM 1905 Acta Math. 29101

[Monthus and Bouchaud (1996)]Monthus C and Bouchaud JP 1996 J. Phys. A 293847

[Montroll and Weiss (1965)]Montroll EW and Weiss GH 1965 J. Math. Phys. 6167

[Montroll and West (1976)]Montroll EW and West BJ 1976 in Fluctuation phenomena edited by Montroll EW and Lebowitz JL (North-Holland, Amsterdam)

[Morgado et al (2002)]Morgado R, Oliveira FA, Batrouni GG and Hansen A 2002 Phys. Rev. Lett. 89 100601

[Naumovets and Zhang (2002)]Naumovets AG and Zhang ZY 2002 Surf. Science 500414

[Nikora et al (2002)]Nikora V, Habersack H, Huber T and McEwan I 2002 Wat. Res. Res. 381081

[Nonnenmacher and Nonnenmacher (1989)]Nonnenmacher TF and Nonnenmacher DJF 1989 Phys. Lett. A 140323 
[Nonnenmacher (1991)]Nonnenmacher TF 1991 in Rheological Modelling: Thermodynamical and Statistical Approaches edited by Casas-Vázquez J and Jou D, Lecture Notes in Physics vol. 381 (Springer-Verlag, Berlin)

[Oddershede et al (2002)] Oddershede L, Dreyer JK, Grego S, Brown S and Berg-Sørensen K Biophys. J. 833152

[Oldham and Spanier (1974)]Oldham KB and Spanier J 1974 The fractional calculus (New York: Academic Press)

[Oldham and Spanier (1972)]Oldham KB and Spanier J 1972 J. Math. Anal. Appl. 39, 655

[Pachepsky et al (2003)]Pachepsky Y, Timlin D and Rawls W 2003 J. Hydrol. 2723

[Peters et al (2002)]Peters O, Hertlein C and Christensen K 2002 Phys. Rev. Lett. 88018701

[Peseckis (1987)]Peseckis FE 1987 Phys. Rev. A 36892

[Pfister and Scher (1977)]Pfister G and Scher H 1977 Phys. Rev. B 152062

[Pfister and Scher (1978)]Pfister G and Scher H 1978 Adv. Phys. 27747

[Picozzi and West (2002)]Picozzi S and West BJ 2002 Phys. Rev. E 66046118

[Plonka (2001)]Plonka A 2001 Dispersive Kinetics (Kluwer, Dordrecht)

[Podlubny (1998)]Podlubny I 1998 Fractional differential equations (San Diego, CA: Academic Press)

[Pottier (2003)]Pottier N 2003 Physica A 317371

[Prosen and Žnidaric (2001)]Prosen T and Žnidaric M 2001 Phys. Rev. Lett. 87114101

[Raberto et al (2002)]Raberto M, Scalas E and Mainardi F 2002 Physica A 314749

[Ramakrishnan and Raj Lakshmi (1987)]Ramakrishnan TV and Raj Lakshmi L (editors) 1987 NonDebye Relaxation in Condensed Matter (World Scientific, Singapore)

[Ramos-Fernandez et al (2003)]Ramos-Fernandez G, Mateos JL, Miramontes O, Cocho G, Larralde H and Ayala-Orozco B 2003 Behav. Ecol. Sociobiol. 55223

[Randriamahazaka et al (2002)]Randriamahazaka H, Noel V and Chevrot C 2002 J. Electoanalyt. Chem. $\mathbf{5 2 1} 107$

[Rangarajan and Ding (2000)]Rangarajan G and Ding M 2000 Fractals 8139

[Rangarajan and Ding (2000a)]Rangarajan G and Ding M 2000 Phys. Rev. E 62120

[Rangarajan and Ding (2003)]Rangarajan G and Ding M 2003 In Processes with Long Range Correlations: Theory and Applications Edited by Rangarajan G and Ding M (New York: SpringerVerlag)

[Re et al (2003)]Re MA, Budde CE and Prato DP 2003 Physica A 3239

[Redner (2001)]Redner S 2001 A Guide to First-Passage Processes (Cambridge: Cambridge University Press)

[Rehfeldt et al (1992)]Rehfeldt KR Boggs JM and Gelhar LW 1992 Wat. Res. Res. 283309

[Reigada et al (2002)]Reigada R, Sarmiento A and Lindenberg K 2002 Physica A 305467

[Ren et al (2003)]Ren FY, Liang JR, Qiu WY, Wang XT, Xu Y and Nigmatullin RR Phys. Lett. A 312187

[Ren et al (2003a)]Ren FY, Liang JR, Qiu WY and Xu Y 2003a J. Phys. A 367533

[Richardson (1926)]Richardson LF 1926 Proc. Roy. Soc. 110709

[Richert (2002)]Richert R 2002 J. Phys. Cond. Math. 14 R703

[Rinn et al (2000)]Rinn B, Maass P and Bouchaud JP Phys. Rev. Lett. 845403

[Rocco and West (1999)]Rocco A and West BF 1999 Physica A 265535

[Ryabov and Puzenko (2002)]Ryabov YE and Puzenko A 2002 Phys. Rev. B 66184201

[Ryabov et al (2003)]Ryabov YE, Nuriel H, Marom G and Feldman Y 2003 J. Polym. Sci. B Polym. Phys. 41217

[Saichev and Zaslavsky (1997)]Saichev AI and Zaslavsky GM 1997 Chaos 7753

[Salman et al (2001)]Salman H, Zbaida D, Rabin Y, Chatenay D and Elbaum M 2001 Proc. Natl. Acad. Sci. U.S.A. 987247

[Samko et al (1993)]Samko SG, Kilbas AA and Marichev OI 1993 Fractional Integrals and Derivatives, Theory and Applications (New York: Gordon and Breach)

[Samorodnitsky and Taqqu (1994)]Samorodnitsky G and Taqqu MS 1994 Stable non-gaussian random 
processes: Stochastic models with infinite variance (New York:Chapman and Hall)

[Saxena and Saigo (2001)]Saxena RK and Saigo M 2001 J. Fract. Calc. 1989

[Scafetta and West (2003)]Scafetta N and West BJ 2003 Phys. Rev. Lett. 90248701

[Scafetta et al (2002)]Scafetta N, Latora V and Grigolini P 2002 Phys. Rev. E 66031906

[Scalas et al (2000)]Scalas E, Gorenflo R and Mainardi F 2000 Physica A 284376

[Schaufler et al (1999)]Schaufler S, Schleich WP and Yakovlev VP 1999 Laser Phys. 9277

[Schaufler et al (1999a)]Schaufler S, Schleich WP and Yakovlev VP 1999 Phys. Rev. Lett. 833162

[Scher and Montroll (1975)]Scher H and Montroll EW 1975 Phys. Rev. B 122455

[Scher et al (2002)]Scher H, Margolin G, Metzler R, Klafter J and Berkowitz B 2002 Geophys. Res. Lett. 291061

[Scher et al (2002a)]Scher H, Margolin G and Berkowitz B 2002 Chem. Phys. 284349

[Schertzer et al (2001)]Schertzer D, Larcheveque M, Duan J, Yanovsky VV and Lovejoy S $2001 \mathrm{~J}$. Math. Phys. 42200

[Schiessel et al (1995)]Schiessel H, Metzler R, Blumen A and Nonnenmacher TF 1995 J. Phys. A 28 6567

[Schiessel et al (1997)]Schiessel H, Sokolov IM and Blumen A 1997 Phys. Rev. E 562390

[Schneider and Wyss (1989)]Schneider WR and Wyss W 1989 J. Math. Phys. 272782

[Schneider et al (1999)]Schneider U, Lunkenheimer P, Brand R and Loidl A 1999 Phys. Rev. E 596924

[Schrödinger (1915)]Schrödinger E 1915 Physikal. Zeitschr. 16289

[Schulz (2002)]Schulz M 2002 Phys. Lett. A 298105

[Schulz and Reineker (2002)]Schulz M and Reineker P 2002 Chem. Phys. 284331

[Seki et al (2003)]Seki K, Wojcik M and Tachiya M 2003 J. Chem. Phys. 1192165

[Seki et al (2003a)]Seki K, Wojcik M and Tachiya M 2003a J. Chem. Phys. 1197525

[Seshadri and West (1982)]Seshadri V, West BJ. 1982. Proc. Natl. Acad. Sci. U.S.A. 794501

[Shimizu et al (2001)]Shimizu KT, Neuhauser RG, Leatherdale CA, Empedocles SA, Woo WK and Bawendi MG 2001 Phys. Rev. B 63205316

[Shlesinger et al (1993)]Shlesinger MF, Zaslavsky GM and Klafter J 1993 Nature 36331

[Shlesinger and Klafter (1990)]Shlesinger MF and Klafter J 1990 in Biological motion edited by Alt W and Hoffmann GH, Lecture Notes in Biomathematics (Springer-Verlag, Berlin)

[Shushin (2001)]Shushin AI 2001 Phys. Rev. E 64051108

[Shushin (2003)]Shushin AI 2003 Phys. Rev. E 67061107

[Simonsen et al (2002)]Simonsen I, Jensen MH and Johansen A 2002 Euro. Phys. J. B 27583

[Sinai (1982)]Sinai YG 1982 Theor. Prob. Appl. 27256

[Slutsky et al (2003)]Slutsky M, Kardar M and Mirny LA 2003 E-print cond-mat/0310008

[Slutsky and Mirny (2004)]Slutsky M and Mirny LA 2004 E-print cond-mat/04

[So and Liu (2004)]So F and Liu KL 2004 Physica A 331378

[Sokolov ()]Sokolov IM, subordination *****

[Sokolov and Belik (2003)]Sokolov IM and Belik VV 2003 Physica A 33046

[Sokolov and Metzler (2003)]Sokolov IM and Metzler R 2003 Phys. Rev. E 67 010101(R)

[Sokolov et al (2001)]Sokolov IM, Klafter J and Blumen A 2001 Phys. Rev. E 64021107

[Sokolov et al (2002)]Sokolov IM, Klafter J and Blumen A 2002 Physics Today 55(11) 48

[Sokolov (2002)]Sokolov IM 2002 Phys. Rev. E 66041101

[Sokolov et al (1997)]Sokolov IM, Mai J and Blumen A 1997 Phys. Rev. Lett. 79857

[Sokolov et al (2001)]Sokolov IM, Blumen A and Klafter J 2001 Physica A 302268

[Sparre Andersen (1953)]Sparre Andersen E 1953 Math. Scand. 1263

[Sparre Andersen (1954)]Sparre Andersen E 1954 Math. Scand. 2195

[Spitzer (1960)]Spitzer *****

[Srivastava et al (1982)]Srivastava HM, Gupta KC and Goyal SP 1982 The H-functions of one and two variables with applications (New Delhi: South Asian Publishers)

[Srivastava and Saxena (2001)]Srivastava HM and Saxena RK 2001 Appl. Math. Comput. 1181

[Stanislavsky (2004)]Stanislavsky AA 2004 Theor. Math. Phys. 138, 418 
[Stanislavsky (2003)]Stanislavsky AA 2003 Phys. Rev. E 67021111

[Stapf (2002)]Stapf S 2002 Chem. Phys. 284369

[Stapf (1995)]Stapf S 1995 Phys. Rev. Lett. 752855

[Sudicky (1986)]Sudicky EA 1986 Wat. Res. Res. 222069

[Sung et al (2002)]Sung YC, Barkai E, Silbey RJ and Lee S 2002 J. Chem. Phys. 1162338

[Takayasu (1990)]Takayasu H 1990 Fractals in the physical sciences (Manchester University Press, Manchester)

[Talkner (2001)]Talkner P 2001 Phys. Rev. E 64061101

[Tamura et al (2002)]Tamura K, Hidaka Y, Yusuf Y and Kai S 2002 Physica A 306157

[Tofighi (2003)]Tofighi A 2003 Physica A 32929

[Tolic-Nørrelykke et al (2003)] Tolic-Nørrelykke IM, Munteanu E-L, Thon G, Oddershede L and BergSørensen K 2003 Subdiffusion and Local Viscoelasticity in Living Cells Preprint

[Tsallis and Lenzi (2002)]Tsallis C and Lenzi EK 2002 Chem. Phys. 284341

[Tseng and Wirtz (2002)]Tseng Y and Wirtz D 2001 Biophys. J. 811643

[Uchaikin (2003)]Uchaikin VV 2003 Int. J. Theor. Phys. 42121

[Uchaikin (2002)]Uchaikin VV 2002 Chem. Phys. 284507

[van Kampen (1981)]van Kampen NG 1981 Stochastic Processes in Physics and Chemistry (Amsterdam: North-Holland)

[Vargas et al (2003)] Vargas WL, Murcia JC, Palacio LE and Dominguez DM 2003 Phys. Rev. E 68 021302

[Vega et al (2002)]Vega JL, Guantes R and Miret-Artes S 2002 J. Phys. Cond. Math. 146193

[Vicsek (1991)]Vicsek T 1989 Fractal growth phenomena (Singapore: World Scientific)

[Visser and Thygesen (2003)]Visser AW and Thygesen UH 2003 J. Plankton Res. 251157

[Viswanathan et al (1996)] Viswanathan GM, Afanasyev V, Buldyrev SV, Murphy EJ, Prince PA and Stanley HE 1996 Nature $\mathbf{3 8 1} 413$

[Viswanathan et al (1999)] Viswanathan GM, Buldyrev SV, Havlin S, da Luz MGE, Raposo MP and Stanley HE 1999 Nature 401911

[Vlad and Ross (2002)]Vlad MO and Ross J 2002 Phys. Rev. E 66061908

[Vlad et al (2000)]Vlad MO, Ross J and Schneider FW 2000 Phys. Rev. E 621743

[Vlad et al (1998)]Vlad MO, Metzler R and Ross J 1998 Phys. Rev. E 576497

[Vlad et al (1996)]Vlad MO, Metzler R, Nonnenmacher TF and Mackey MC 1996 J. Math. Phys. 37 2279

[von Smoluchowski (1916)]von Smoluchowski M 1916 Physkal. Zeitschr. 17557

[von Smoluchowski (1916a)]von Smoluchowski M 1916 Physkal. Zeitschr. 17585

[Wang et al (1994)]Wang KG, Dong LK, Wu XF, Zhu FW and Ko T 1994 Physica A 20353

[Wang and Tokuyama (1999)]Wang KG and Tokuyama M 1999 Physica A 265341

[Weeks and Weitz (2002)]Weeks ER and Weitz DA 2002 Chem. Phys. 284361

[Weeks et al (2000)]Weeks ER, Crocker JC, Levitt AC, Schofield A and Weitz DA 2000 Science 287 627

[West et al (1997)]West BJ, Grigolini P, Metzler R and Nonnenmacher TF 1997 Phys. Rev. E 5599

[West and Nonnenmacher (2001)]West BJ and Nonnenmacher TF 2001 Phys. Lett. A 278255

[Wolf (1979)]Wolf KB 1979 Integral Transforms in Science and Engineering (New York: Plenum Press)

[Wong et al (2003)]Wong IY, Gardel ML, Reichman DR, Weeks ER, Valentine MT, Bausch AR and Weitz DA 2003 Anomalous Diffusion Probes Microstructure Dynamics of Entangled F-actin Networks Preprint

[Yang and Xie (2002)]Yang H and Xie XS 2002 J. Chem. Phys. 11710965

[Yang et al (2003)] Yang H, Luo G, Karnchanaphanurach P, Louie T-M, Rech I, Cova S, Xun L and Xie XS 2003 Science 302262

[Yuste and Lindenberg (2004)]Yuste SB and Lindenberg K 2004 Phys. Rev. E at press

[Yuste and Lindenberg (2001)]Yuste SB and Lindenberg K 2001 Phys. Rev. Lett. 87118301

[Yuste and Lindenberg (2002)]Yuste SB and Lindenberg K 2002 Chem. Phys. 284169 
[Zaburdaev (2003)]Zaburdaev VY and Chukbar KV 2003 JETP Lett. 77551

[Zahran et al (2003)]Zahran MA, Abulwafa EM, Elwakil SA 2003 Physica A 323237

[Zaslavsky (1999)]Zaslavsky GM 1999 Phys. Today 52(8) 39

[Zaslavsky (2002)]Zaslavsky GM 2002 Phys. Rep. 371461

[Zhou et al (2002)]Zhou J-L, Sun Y-S and Zhou L-Y 2002 Celest. Mechan. Dynam. Astron. 84409

[Zhou and Sun (2001)]Zhou J-L and Sun Y-S 2001 Phys. Lett. A 287217

[Zumofen et al (1990]Zumofen, G, Klafter J and Blumen A 1990 Phys. Rev. A 424601

[Zumofen and Klafter (1993)]Zumofen G and Klafter J 1993 Phys. Rev. E 47851

[Zumofen et al (1996)]Zumofen G, Klafter J and Shlesinger MF 1996 Chem. Phys. 21289

[Zumofen et al (1996a)]Zumofen G, Klafter J and Shlesinger MF 1996a Phys. Rev. Lett. 772830

[Zumofen and Klafter (1995)]Zumofen G and Klafter J 1995 Phys. Rev. E 512805

[Zumofen and Klafter (1995a)]Zumofen G and Klafter J 1995a Phys. Rev. E 511818

[Zumofen and Klafter (1994)]Zumofen G and Klafter J 1994 Chem. Phys. Lett. 219303 